LBL --31255

DE92 009135

\title{
Release Rates from Partitioning and Transmutation Waste Packages
}

\author{
William W.-L. Lee and Jor-Shan Choi ${ }^{\dagger}$ \\ ${ }^{*}$ Earth Sciences Division \\ Lawrence Berkeley Laboratory \\ University of California \\ Berkeley, California 94720 \\ $\dagger_{\text {Lawrence Livermore National Laboratory }}$ \\ University of California \\ Livermore, California 94550
}

December 1991

This document is

PUBLICLY RELEASABLE

2 Stenk

Authorizing Official

Date: $\quad 3 \cdot 15 \cdot 07$

This work was supported in part by the Director, Office of Civilian Radioactive Waste Management, Yucca Mountain Site Characterization Office, of the U.S. Department of Energy under Contract No. DE-AC0376 SF00098. 


\section{DISCLAIMER}

This report was prepared as an account of work sponsored by an agency of the United States Government. Neither the United States Government nor any agency Thereof, nor any of their employees, makes any warranty, express or implied, or assumes any legal liability or responsibility for the accuracy, completeness, or usefulness of any information, apparatus, product, or process disclosed, or represents that its use would not infringe privately owned rights. Reference herein to any specific commercial product, process, or service by trade name, trademark, manufacturer, or otherwise does not necessarily constitute or imply its endorsement, recommendation, or favoring by the United States Government or any agency thereof. The views and opinions of authors expressed herein do not necessarily state or reflect those of the United States Government or any agency thereof. 


\section{DISCLAIMER}

Portions of this document may be illegible in electronic image products. Images are produced from the best available original document. 


\section{CONTENTS}

Abstract . . . . . . . . . . . . . . . . . . . . . . . . . . . . . . . . 1

1. Introduction . . . . . . . . . . . . . . . . . . . . . . . . . . . . . . . . . . . 1

2. Need for Evaluating the Benefits of Partitioning and Transmutation . . . . . . . . . . . . . . 1

3. An Equal Energy Production Comparison . . . . . . . . . . . . . . . . . . . . . . . . . . 2

4. Waste Characteristics and Inventories . . . . . . . . . . . . . . . . . . . . . . . . . . . 4

5. Calculation of Release Rates . . . . . . . . . . . . . . . . . . . . . . . . . . . . . . . 10

5.1 The Wet-Drip Water-Contact Mode . . . . . . . . . . . . . . . . . . . . . . . . 33

5.2 Parameters Adopted for Calculating Release Rates . . . . . . . . . . . . . . . . . . . 33

5.3 Calculated Release Rates . . . . . . . . . . . . . . . . . . . . . . . . . . . . . 35

6. Conclusions . . . . . . . . . . . . . . . . . . . . . . . . . . . . . . . . . . . . . . 49

References . . . . . . . . . . . . . . . . . . . . . . . . . . . . . . . 53

Appendix 
Table 1. Waste packages from pyro-processing of LWR spent fuel. . . . . . . . . . . . . . . . . 8

Table 2. Waste packages from aqueous processing of LWR spent fuel. . . . . . . . . . . . . . . . 9

Table 3. Waste packages from pyro-processing of ALMR fuel. . . . . . . . . . . . . . . . . . . 10

Table 4. Inventory of a Spent-Fuel Waste Package. . . . . . . . . . . . . . . . . . . . . . . 11

Table 5. Inventory of an A1-1,2 Package. . . . . . . . . . . . . . . . . . . . . . . . . . 13

Table 6. Inventory of an A1-3 Package. . . . . . . . . . . . . . . . . . . . . . . 15

Table 7. Inventory of an A1-4 Package. . . . . . . . . . . . . . . . . . . . . . . . 16

Table 8. Inventory of an A1-6 Package. . . . . . . . . . . . . . . . . . . . . . . . . . . 17

Table 9. Inventory of an A1-7 Package. . . . . . . . . . . . . . . . . . . . . . . . 18

Table 10. Inventory of an A1-8 Package. . . . . . . . . . . . . . . . . . . . . . . . . . . 19

Table 11. Inventory of an A3-2,5 Package. . . . . . . . . . . . . . . . . . . . . 21

Table 12. Inventory of an A3-4 Package. . . . . . . . . . . . . . . . . . . . . . . . . . . 23

Table 13. Inventory of a B1-2 Package. . . . . . . . . . . . . . . . . . . . . . . . . . . . 24

Table 14. Inventory of a B1-3 Package. . . . . . . . . . . . . . . . . . . . . . . . 26

Table 15. Inventory of a B1-4 Package. . . . . . . . . . . . . . . . . . . . . . . . . . 27

Table 16. Inventory of the A1 Process. . . . . . . . . . . . . . . . . . . . . . . . . . . . 29

Table 17. Inventory of the A3 Process. . . . . . . . . . . . . . . . . . . . . . . . . . . . 30

Table 18. Inventory of the B1 Process. . . . . . . . . . . . . . . . . . . . . . . . . . 31

Table 19. Release Mechanisms . . . . . . . . . . . . . . . . . . . . . . . . . 34

Table 20. Solubility Data . . . . . . . . . . . . . . . . . . . . . . . . . . . . 34

\section{FIGURES}

Figure 1. Equal Energy Production Schemes . . . . . . . . . . . . . . . . . . . . . 3

Figure 2. Waste streams from pyrochemical processing of LWR spent fuel. . . . . . . . . . . . . 5

Figure 3. Waste streams from aqueous processing of LWR spent fuel. . . . . . . . . . . . . . . . 6

Figure 4. Waste streams from pyrochemical processing of ALMR spent fuel. . . . . . . . . . . . . 7

Figure 5. Fractional release rates of selected species from waste package A3-2,5 . . . . . . . . . . 36

Figure 6. Fractional release rates of selected species from waste package B1-4 . . . . . . . . . . . 37

Figure 7. Fractional release rates of Tc-99 from LWR spent fuel and reprocessed wastes . . . . . . 38

Figure 8. Release rates of Tc-99 from LWR spent fuel and reprocessed wastes . . . . . . . . . . . . 39

Figure 9. Fractional release rates of Cs-135 from LWR spent fuel and reprocessed wastes . . . . . . 41

Figure 10. Release rates of Cs-135 from LIVR spent fuel and reprocessed wastes . . . . . . . . . . 42

Figure 11. Fractional release rates of I-129 from LWR spent fuel and reprocessed wastes . . . . . . 43

Figure 12. Fractional release rates of $\mathrm{Np}-237$ from reprocessed wastes . . . . . . . . . . . . . . . 44

Figure 13. Fractional release rates of uranium species from LWR spent fuel and reprocessed wastes . 45

Figure 14. Fractional release rates of plutonium from LWR spent fuel and reprocessed wastes . . . . 46

Figure 15. Release rates of plutonium from LWR spent fuel and reprocessed wastes . . . . . . . . 47

Figure 16. Release rates of $\mathrm{I}-129$ from Schemes $1,2 \mathrm{a}$ and $2 \mathrm{~b}$. . . . . . . . . . . . . . . . . . . . . . 48

Figure 17. Release rates of Tc-99 from Schemes 1, 2a and $2 \mathrm{~b}$. . . . . . . . . . . . . . . . . . 50

Figure 18. Release rates of $\mathrm{Np}-237$ from Schemes $1,2 \mathrm{a}$ and $2 \mathrm{~b}$. . . . . . . . . . . . . . . . . 51

Figure 19. Release rates of $\mathrm{Pu}$ isotopes from Schemes $1,2 \mathrm{a}$ and $2 \mathrm{~b}$. . . . . . . . . . . . . . . 52 


\begin{abstract}
Partitioning the actinides in light-water reactor spent fuel and transmuting them in actinide-burning liquid-metal reactors has been proposed as a potential method of reducing the public risks from geologic disposal of nuclear waste. As a first step towards quantifying the benefits for waste disposal of actinide burning, we have calculated the release rates of key radionuclides from waste packages resulting from actinide burning, and compare them with release rates from LWR spent fuel destined for disposal at the potential repository at Yucca Mountain. The wet-drip water-contact mode has been used. Analytic methods and parameter values are very similar to those used for assessing Yucca Mountain as a potential repository. Once released, the transport characteristics of radionuclides will be largely determined by site geology. For the most important nuclides such as I-129 and Tc-99, which are undiminished by actinide-burning reactors, it is not surprising that actinide buming offers little reduction in releases. For important actinides such as Np-237 and Pu isotopes, which are reduced in inventory, the releases are not reduced because the release rates are proportional to solubility, rather than inventory.
\end{abstract}

\title{
1. Introduction
}

Partitioning the actinides in light-water reactor (LWR) spent fuel and transmuting them in actinide-burning liquid-metal reactors (ALMRs) has been put forth as a potential method of reducing the public risks from geologic disposal of nuclear waste. However, the real benefits of such partitioning and transmutation for waste disposal have not been analysed. Efforts to quantify these benefits are now underway. This report provides the following

a. Elucidation of an equal energy produced basis of comparison,

b. Characteristics and inventories of exotic waste packages from aqueous and pyro-reprocessing schemes,

c. Release rates of selected radionuclides that are likely to travel to the accessible environment from the potential repository at Yucca Mountain.

\section{Need for Evaluating the Benefits of Partitioning and Transmutation}

The slow pace of technological progress as well as seemingly overwheming public opposition to geologic disposal of spent nuclear fuel has brought forth the concepts of partitioning and transmutation to reduce the risks to the public of waste disposal. Spent nuclear fuel can be reprocessed, and the waste can be partitioned or separated into elemental fractions which can then be transmuted into stable or short-lived isotopes by bombardment with neutrons. Partitioning involves chemical processes and can be done in a reprocessing facility. Transmutation can be accomplished in accelerators or reactors. Actinide burning is the concept of using the transuranics in LWR spent fuel in a liquid-metal fast reactor to generate electricity as well as perform transmutation.

While the technology for partitioning and transmutation was developed in the 1970's and 1980's, the waste disposal community has always regarded the benefits from partitioning and transmutation to be marginal, compared to the magnitude of the undertaking [IAEA 1982]. However, recent difficulties at Yucca Mountain [Broad 1990] have given new impetus to partitioning and transmutation. 
The main claimed benefits of partitioning and transmutation are

- partitioning and transmutation reduce health risk to future generations.

- partitioning and transmutation reduce the heat placed in the repository.

- partitioning and transmutation ease the licensing of a repository.

- partitioning and transmutation make the repository more acceptable to the public.

Only the first two claims can be evaluated quantitatively. In this report we give the inventories of major nuclides in the repository from various schemes, and calculate the release of the nuclides from waste packages. The release rates allow total systems analysts to calculate risks to individuals.

\section{An Equal Energy Production Comparison}

In this Section we describe the basis of comparing the reference case of spent-fuel disposal at Yucca Mountain with two variants of partitioning and transmutation.

The schemes being compared are shown in Figure 1.

Scheme 1 is disposal of light-water reactor spent-fuel.

In Scheme 2, the geologic repository receives waste from the reprocessing of LWR and the reprocessing of ALMR fuel. In order to provide initial fuel, reloads and makeup for actinide-burning liquid-metal reactors, light-water reactor (LWR) spent-fuel is reprocessed, by either conventional aqueous reprocessing technology, the PUREX process [Benedict, Pigford and Levi 1981], or pyrochemical reprocessing technology under development [Lineberry and Phipps 1989]. We shall take the 63000 MTIHM of LWR spent fuel destined for the first repository and reprocess for use in the General Electric PRISM reactor [Thompson 1991], the reference U.S. Department of Energy advanced liquid-metal reactor. We make the following assumptions about the ALMR's

- Nine modules of PRISM produce 1395 MWe

- The reactors have 40 years of economic life

- The ALMR's have a capacity factor of 0.8 and conversion ratio of 0.76

In Scheme 2a, the LWR spent fuel is reprocessed with pyrochemical processes, and the ALMR fuel processed with pyrochemical processes. We shall designate waste streams in the pyro-processing of LWR fuel as A1-x, and waste streams in the pyro-processing of ALMR fuel as A3-x [Thompson and Taylor 1991].

In Scheme 2b, the LWR spent fuel is reprocessed with aqueous processes, and the ALMR fuel processed with pyrochemical processes. We shall designated waste streams in the aqueous processing of LWR fuel as B1-x, and waste streams in the pyro-processing of ALMR fuel as A3-x [Thompson and Taylor 1991]. 


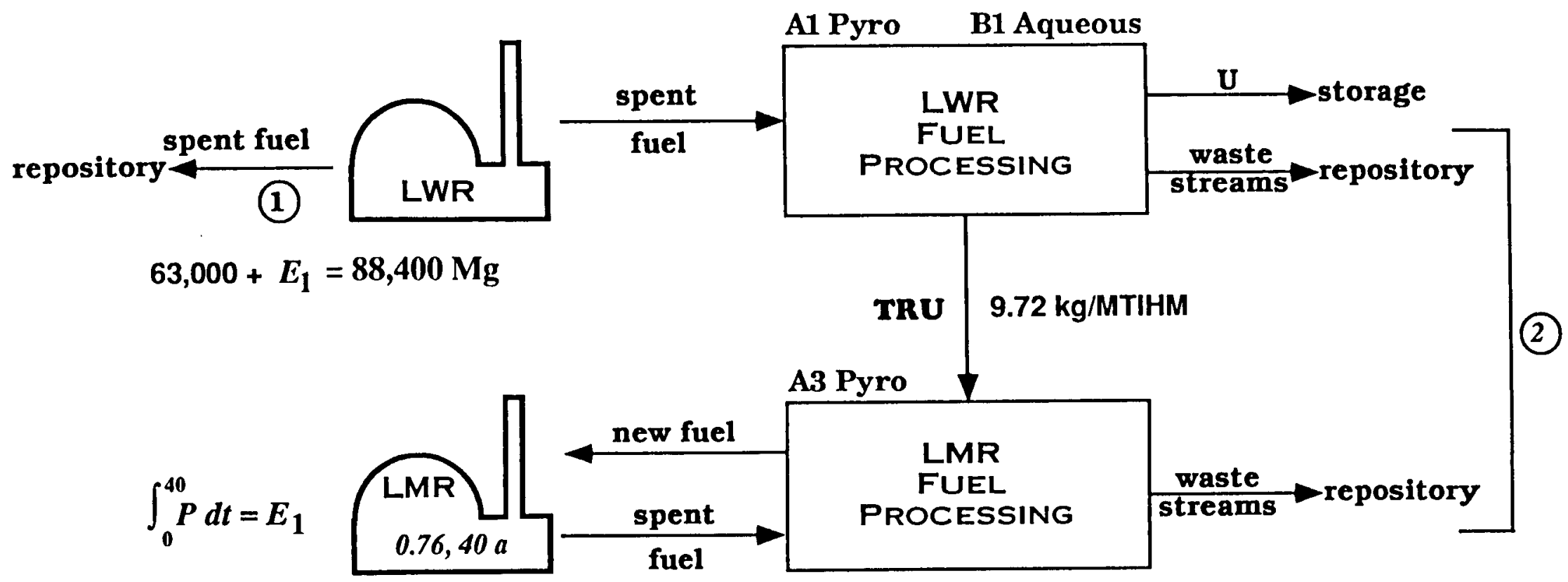

Figure 1. Equal Energy Production Comparison 
For the following calculations, we use $33,000 \mathrm{MWd} /$ ton burnup fuel from pressurized water reactors as the reference case. With each $\mathrm{Mg} U$ or MTIHM of LWR spent fuel giving $9.72 \mathrm{~kg}$ of transuranics, $3878 \mathrm{MTIHM}$ of LWR spent fuel are needed to support one 1395-MWe ALMR, and the 63,000 MTIHM would support about 16 in all. In the course of their economic lives, these 16 ALMR's would produce $9.1 \times 10^{5} \mathrm{MWe}$-a of energy. Thus a repository serving Scheme $2 a$ or $2 b$ would contain the waste of $9.1 \times 10^{5} \mathrm{MWe}$-a of energy plus the waste from the reprocessing of 63,000 MTIHM of LWR spent fuel. For a fair or equal energy produced comparison, we now add to Scheme 1 the equivalent LWR spent fuel that would have resulted from the generation of $9.1 \times 10^{5}$ MWe-a of energy using LWR's, or

$$
63000+9.1 \times 10^{5} \times \frac{28 \mathrm{MTIHM} \mathrm{SF}}{1000 \mathrm{MWe}-\mathrm{a}}=88400 \mathrm{MTIHM}
$$

Therefore the repository serving Scheme 1 should contain 88400 MTIHM for an equal-energy produced comparison.

\section{Waste Characteristics and Inventorles}

Figure 2 shows the waste streams from pyrochemical processing of LWR spent fuel, the A1-x streams. Figure 3 shows the waste streams from aqueous processing of LWR spent fuel, the B1-X streams. Figure 4 shows the waste streams from pyrochemical processing of ALMR spent fuel, the A3-x streams. These figures have been taken from Thompson and Taylor [1991].

Waste characteristics and inventories for the schemes shown in Figure 1 have been studied by Thompson and Taylor [1991], revised by Wilems and Danna [1991] and we revised them further. We use the inventories given by Thompson and Taylor [1991]. We adopted the simplified waste packaging suggested by Wilems and Danna [1991] and their per package thermal limit of $2.5 \mathrm{Kw} /$ package.

These are the major modifications we made.

- In Scheme 2b we considered low-recovery (99.9\%) aqueous processing. The actinide distribution in B1 waste is from the high-recovery (99.999\%) tables by Thompson and Taylor and scaled back to $99.9 \%$. For pyro-processing, we use $99.9 \%$ recovery.

- Where I-129 is considered a gas, we convert it to AgI, a low-solubility compound that is a more leachresistant waste form.

- We put the fuel hardware from ALMR (A3-2) into the electro-refining metal waste (A3-5), which has a copper matrix, forming A3-2,5.

- Gaseous nuclides and short-lived wastes such as A1-5 can be allowed to decay. If disposed in any repository, these species will not affect dose to humans except in human intrusion scenarios. 


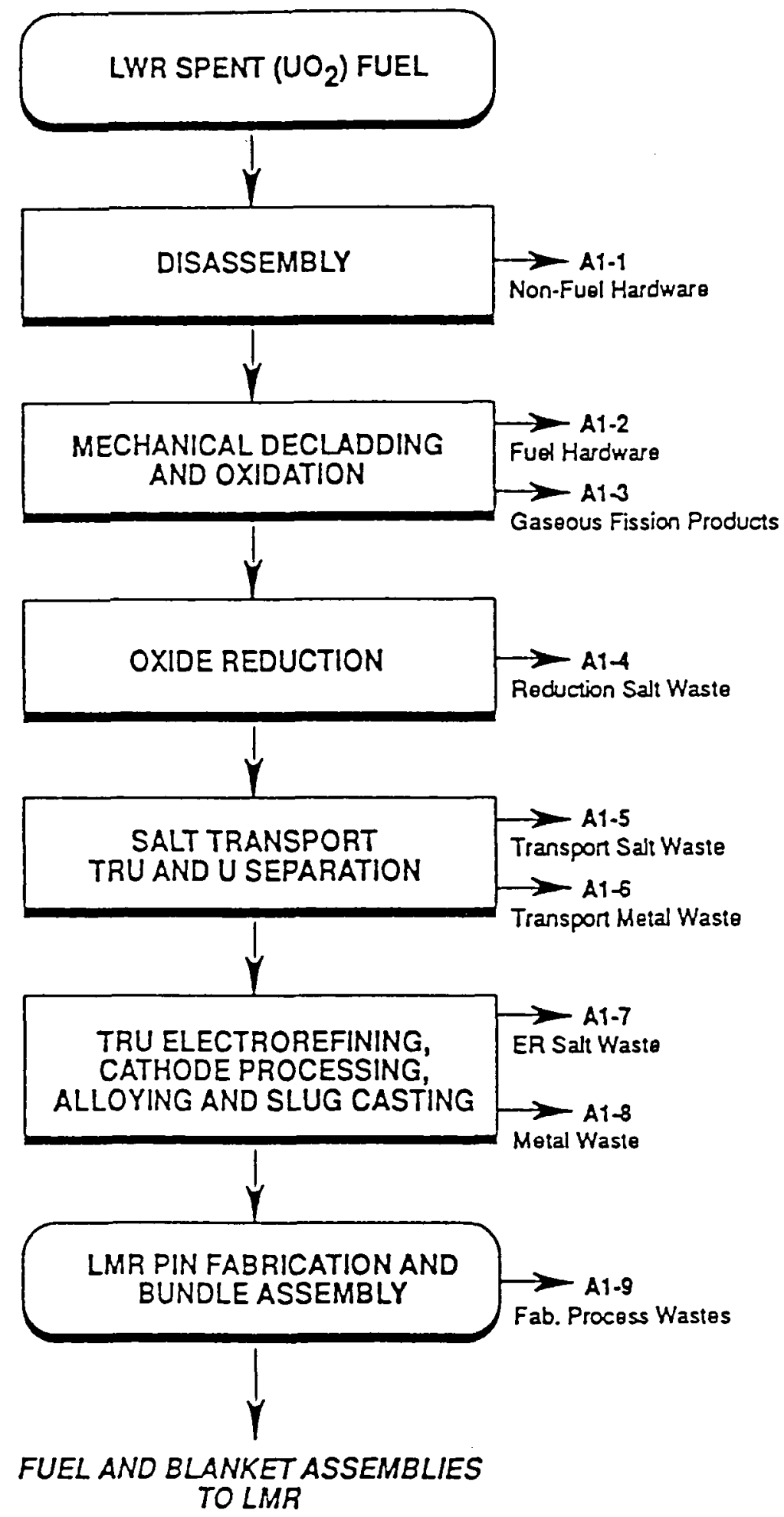

Figure 2. Waste streams from pyrochemical processing of LWR spent fuel.

[from Thompson and Taylor 1991] 


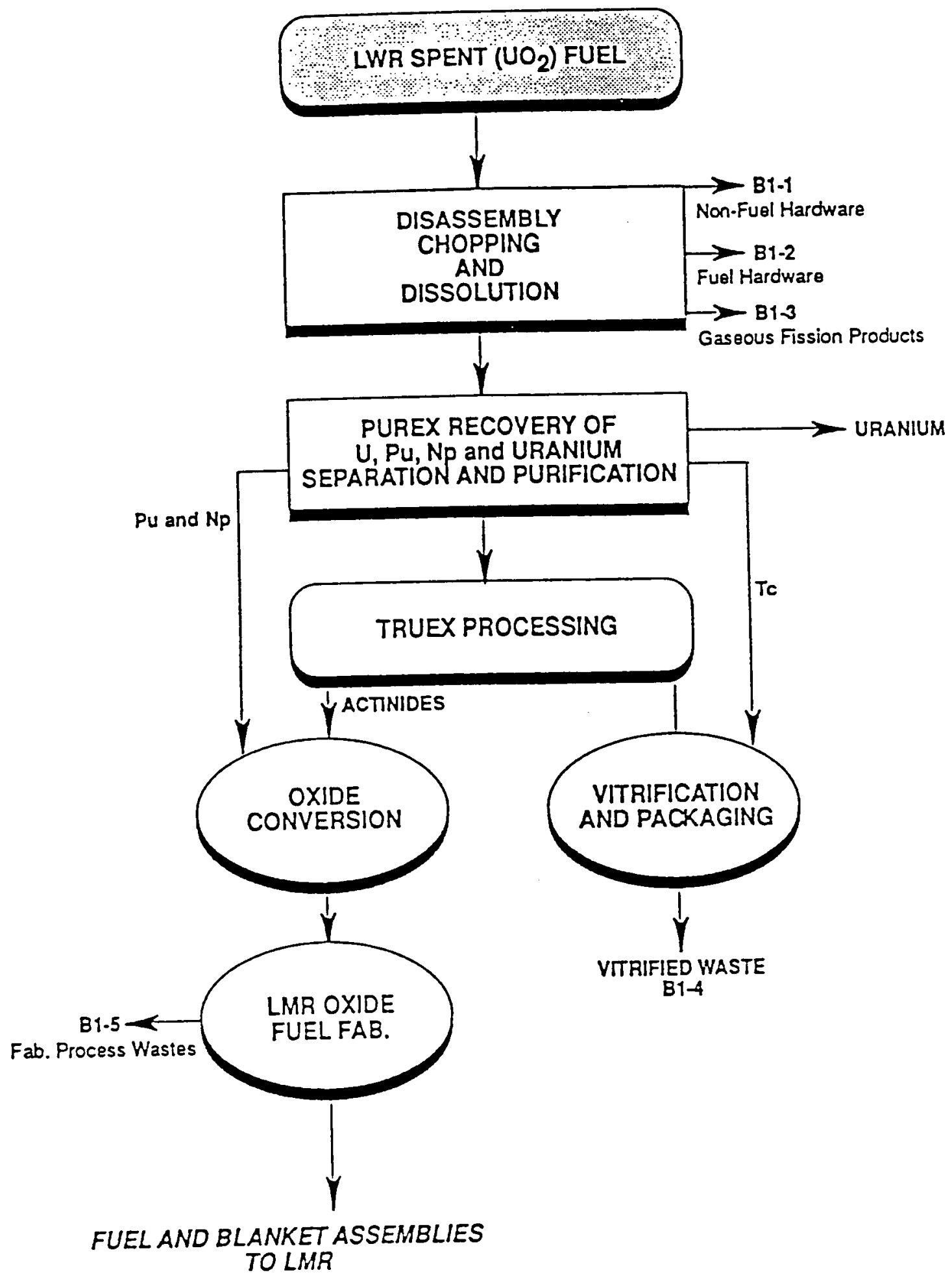

Figure 3. Waste streams from aqueous processing of LWR spent fuel.

[from Thompson and Taylor 1991] 


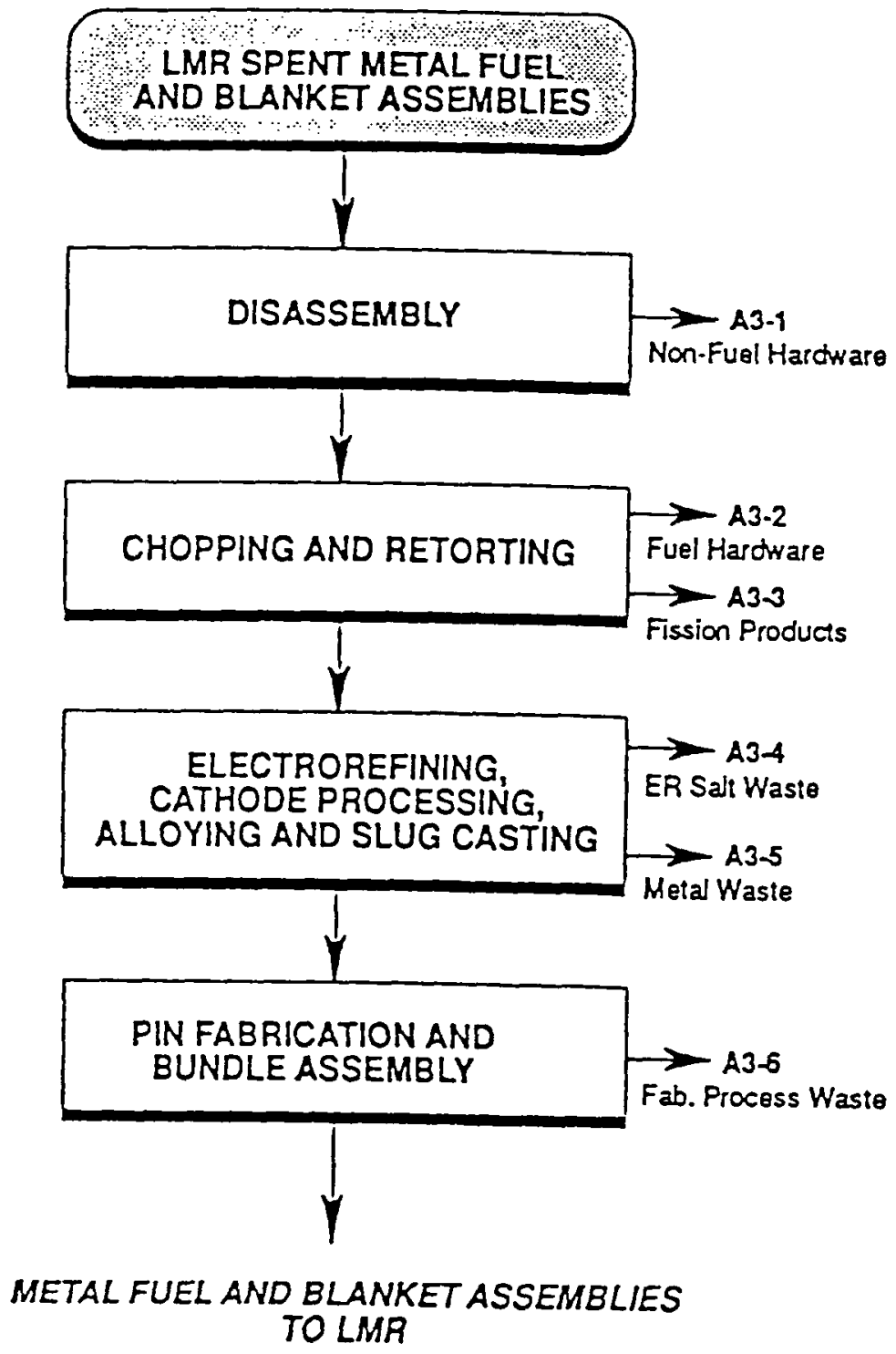

Figure 4. Waste streams from pyrochemical processing of ALMR spent fuel.

[from Thompson and Taylor 1991] 
Table 1. Waste packages from pyro-processing of LWR spent fuel

\begin{tabular}{|c|c|c|c|c|c|c|}
\hline Pyro-processing of LWR SF & A $1-1,2$ & A1-3 & $\mathrm{A} 1-4$ & A1-6 & A $1-7$ & A1-8 \\
\hline Waste Stream & Hardware & Gases & $\begin{array}{c}\text { Reduction } \\
\text { Salt } \\
\end{array}$ & $\begin{array}{c}\text { Transport } \\
\text { Metal } \\
\end{array}$ & $\begin{array}{c}\text { Electro-refining } \\
\text { Salt }\end{array}$ & $\begin{array}{c}\text { Electro-refining } \\
\text { Metal }\end{array}$ \\
\hline Container Type & 6 & 6 & 5 & 6 & 5 & 5 \\
\hline Inside Container Dia (m) & 0.59 & 0.59 & 0.4 & 0.59 & 0.4 & 0.4 \\
\hline Inside Container Ht (m) & 5.0 & 5.0 & 5.0 & 5.0 & 5.0 & 5.0 \\
\hline Inside $X$ Area $\left(\mathrm{m}^{2}\right)$ & 0.273 & 0.273 & 0.125 & 0.273 & 0.125 & 0.125 \\
\hline Inside Container Material & SS & SS & SS & SS & SS & SS \\
\hline Outside Container Dia (m) & 0.66 & 0.66 & 0.47 & 0.66 & 0.47 & 0.47 \\
\hline Outside Container Ht (m) & 5.22 & 5.22 & 5.22 & 5.22 & 5.22 & 5.22 \\
\hline Outside X Area $\left(\mathrm{m}^{2}\right)$ & 0.342 & 0.342 & 0.173 & 0.342 & 0.173 & 0.173 \\
\hline Outside Container Material & SS & SS & SS & SS & SS & SS \\
\hline Waste Volume $\left(\mathrm{m}^{\mathbf{3}}\right)$ & 1.16 & 1.16 & 0.53 & 1.16 & 0.53 & 0.53 \\
\hline Void Volume $\left(\mathrm{m}^{3}\right)$ & 0.624 & 0.624 & 0.37 & 0.624 & 0.37 & 0.37 \\
\hline MATRIX & None & AgI & Zeolite & Copper & Zeolite & Copper \\
\hline $\mathrm{KW} / \mathrm{pkg}$ at 10 years & 0.57 & 0.00126 & 2.08 & 0.19 & 0.35 & 1.45 \\
\hline Number of Containers & 4190 & 5 & 25589 & 2394 & 1646 & 1500 \\
\hline
\end{tabular}


Table 1 shows the waste packages from pyro-processing of LWR spent fuel. Table 2 shows the waste packages from aqueous processing of LWR spent fuel. Table 3 shows the waste packages from pyro-processing of ALMR fuel. In each case, the dimensions, materials, heat output, matrix and number of packages are shown.

Table 2. Waste packages from aqueous processing of LWR spent fuel

\begin{tabular}{|l|c|c|c|}
\hline Waste Stream & B1-2 & B1-3 & B1-4 \\
\hline & Hardware & Gas & Glass \\
\hline Inside Container Diameter $(\mathrm{m})$ & 0.59 & 0.59 & 0.4 \\
\hline Inside Container Height $(\mathrm{m})$ & 5.0 & 5.0 & 5.0 \\
\hline Inside X-Area $\left(\mathrm{m}^{2}\right)$ & 0.273 & 0.273 & 0.125 \\
\hline Inside Container Material & $\mathrm{SS}$ & $\mathrm{SS}$ & $\mathrm{SS}$ \\
\hline Outside Container Diameter $(\mathrm{m})$ & 0.66 & 0.66 & 0.47 \\
\hline Outside Container Height $(\mathrm{m})$ & 5.22 & 5.22 & 5.22 \\
\hline Outside X-Area $\left(\mathrm{m}^{2}\right)$ & 0.342 & 0.342 & 0.173 \\
\hline Outside Container Material & $\mathrm{SS}$ & $\mathrm{SS}$ & $\mathrm{SS}$ \\
\hline Waste Volume $\left(\mathrm{m}^{3}\right)$ & 1.16 & 1.16 & 0.53 \\
\hline Void Volume $\left(\mathrm{m}^{3}\right)$ & 0.624 & 0.624 & 0.37 \\
\hline MATRIX & None & $\mathrm{AgI}$ & Glass \\
\hline Kilowatts/Package & & & \\
\hline at 10 years & 0.57 & 0.00126 & 2.50 \\
\hline Number of Containers & 4191 & 5 & 24550 \\
\hline
\end{tabular}

In this study, we track 33 radionuclides. They have been chosen because of their significance in waste disposal.

These species have one or more of the following characteristics

- Long half life

- High toxicity

- Low sorption

- High heat generation

- Large inventory.

For the spent-fuel waste package [Apted et al. 1991], as well as each of the waste packages identified in Tables 1, 2 and 3, the inventories of the tracked radionuclides are given, at 10, 100,300,1000,5000 and 10,000 years after emplacement, in Tables 4 through 15 . This data are the basis for calculating release rates and estimating the impact of inadvertent human intrusion. 
Table 3. Waste packages from pyro-processing of ALMR fuel

\begin{tabular}{|c|c|c|}
\hline Waste Stream & A3-4 & A3-2,5 \\
\hline & $\begin{array}{c}\text { Electro-refining } \\
\text { Salt }\end{array}$ & $\begin{array}{c}\text { Hardware \& } \\
\text { Electro-refining } \\
\text { Metal }\end{array}$ \\
\hline Inside Container Dia. (m) & 0.59 & 0.59 \\
\hline Inside Container Ht. (m) & 5.0 & 5.0 \\
\hline Inside $\mathrm{X}$-Area $\left(\mathrm{m}^{2}\right)$ & 0.273 & 0.273 \\
\hline Outside Container Dia. (m) & 0.66 & 0.66 \\
\hline Outside Container Ht. (m) & 5.22 & 5.22 \\
\hline Outside X-Area $\left(\mathrm{m}^{2}\right)$ & 0.342 & 0.342 \\
\hline Container Material & SS & SS \\
\hline Waste Volume $\left(\mathrm{m}^{3}\right)$ & 1.16 & 1.16 \\
\hline Void Volume $\left(\mathrm{m}^{3}\right)$ & 0.624 & 0.624 \\
\hline MATRIX & Zeolite & Copper \\
\hline $\begin{array}{l}\text { Kilowatts/Package } \\
\text { at } 10 \text { years }\end{array}$ & 0.70 & 1.84 \\
\hline Number of Containers & 16880 & 5114 \\
\hline
\end{tabular}

Inventories of waste packages have been provided for 10,100,300, 1000, 5000 and 10,000 years after emplacement for evaluating the possibility of human intrusion. If a driller were to bring an entire package either to the ground surface or to the water table, information on the inventories is needed.

When the inventories of individual packages are multiplied by the number of packages, one obtains the total radioactivity for that stream, and when the streams are summed, the totals for the process. Total inventories for the $A 1, A 3$ and $B 1$ streams are given in Tables 16 through 18.

\section{Calculation of Release Rates}

We assume that waste from LWR and ALMR cycles will be placed in the potential repository at Yucca Mountain. Current design calls for vertically placed waste containers to be surrounded by an air gap. Although the waste package is generally not seen as the primary barrier for nuclear waste isolation, it must in fact meet specific regulatory requirements. In 10 CFR 60.113(a)(1)(ii)(B), the U. S. Nuclear Regulatory Commission requires that the release rate of any radionuclide from the engineered barrier system following the containment period shall not exceed one part in 100,000 per year of the inventory of that radionuclide calculated to be present at 1,000 years following permanent closure. For low-inventory radionuclides, those 
Table 4. Inventory of a LWR Spent Fuel Waste Package, 1 of 2

\begin{tabular}{|c|c|c|c|c|c|c|c|c|c|}
\hline Nuclide & Lambda & & Ci/pkg & at & year & & & stream & total \\
\hline 42095 & (ia) & 10 & 100 & 300 & 1000 & 5000 & 10000 & Ci at 10 a & $\mathrm{Ci}$ at 1000 a \\
\hline$C-14$ & $1.21 \mathrm{E}-04$ & $3.26 \mathrm{E}+00$ & $3.22 E+00$ & $3.14 E+00$ & $2.89 E+00$ & $1.78 E+00$ & $9.72 \mathrm{E}-01$ & $1.37 E+05$ & $1.22 E+05$ \\
\hline $\mathrm{Ni}-59$ & $9.24 \mathrm{E}-06$ & $1.08 E+01$ & $1.08 \mathrm{E}+01$ & $1.08 E+01$ & $1.07 E+01$ & $1.03 E+01$ & $9.86 \mathrm{E}+00$ & $4.55 E+05$ & $4.51 E+05$ \\
\hline$S e-79$ & $1.07 \mathrm{E}-05$ & $8.61 \mathrm{E}-01$ & $8.60 \mathrm{E}-01$ & $8.58 \mathrm{E}-01$ & $8.52 E-01$ & $8.16 \mathrm{E}-01$ & 7.74E-01 & $3.62 E+04$ & $3.59 E+04$ \\
\hline$S x-90$ & $2.43 E-02$ & $1.20 E+05$ & $1.35 \mathrm{E}+04$ & $1.04 \mathrm{E}+02$ & 4.20E-06 & $2.36 \mathrm{E}-48$ & $3.64 \mathrm{E}-101$ & $5.06 E+09$ & $1.77 \mathrm{E}-01$ \\
\hline$Y-90$ & $9.48 \mathrm{E}+01$ & $1.20 \mathrm{E}+05$ & $0.00 E+00$ & $0.00 E+00$ & $0.00 E+00$ & $0.00 E+00$ & $0.00 E+00$ & $5.06 \mathrm{E}+09$ & $0.00 E+00$ \\
\hline $2 x-93$ & 4.53E-07 & $4.05 E+00$ & $4.05 E+00$ & $4.05 E+00$ & $4.05 \mathrm{E}+00$ & $4.04 E+00$ & $4.03 E+00$ & $1.71 E+05$ & $1.71 E+05$ \\
\hline $\mathrm{Nb}-94$ & $3.41 E-05$ & $2.69 E+00$ & $2.68 \mathrm{E}+00$ & $2.66 E+00$ & $2.60 E+00$ & $2.27 E+00$ & $1.91 E+00$ & $1.13 E+05$ & $1.09 E+05$ \\
\hline Tc-99 & $3.29 E-06$ & $2.75 \mathrm{E}+01$ & $2.75 \mathrm{E}+01$ & $2.75 E+01$ & $2.74 E+01$ & $2.71 E+01$ & $2.66 \mathrm{E}+01$ & $1.16 E+06$ & $1.15 E+06$ \\
\hline$P d-107$ & $1.07 E-07$ & 2.35E.01 & $2.35 \mathrm{E}-01$ & 2.35E-01 & 2.35E-01 & $2.35 \mathrm{E}-01$ & $2.35 E-01$ & $9.90 E+03$ & $9.90 E+03$ \\
\hline $5 n-126$ & $6.93 E-06$ & $1.63 E+00$ & $1.63 E+00$ & $1.63 E+00$ & $1.62 E+00$ & $1.57 \mathrm{E}+00$ & $1.52 E+00$ & $6.86 E+04$ & $6.81 E+04$ \\
\hline$I-129$ & $4.41 \mathrm{E}-08$ & $6.62 \mathrm{E}-02$ & $6.61 E-02$ & $6.61 \mathrm{E}-02$ & $6.61 \mathrm{E}-02$ & $6.61 \mathrm{E}-02$ & $6.61 \mathrm{E}-02$ & $2.78 \mathrm{E}+03$ & $2.78 E+03$ \\
\hline $\mathrm{Cs}-135$ & 3.01E-07 & $7.25 \mathrm{E}-01$ & $7.24 \mathrm{E}-01$ & $7.24 E-01$ & 7.24E-01 & $7.23 E-01$ & $7.22 \mathrm{E}-01$ & $3.05 E+04$ & $3.05 E+04$ \\
\hline Cs-137 & $2.31 E-02$ & $1.72 E+05$ & $2.16 \mathrm{E}+04$ & $2.12 E+02$ & 2.01E-05 & $1.46 \mathrm{E}-45$ & $9.85 E-96$ & $7.26 \mathrm{E}+09$ & $8.45 E-01$ \\
\hline Sm-151 & $7.70 \mathrm{E}-03$ & $6.95 E+02$ & $3.48 E+02$ & $7.45 E+01$ & $3.39 E-01$ & $1.42 \mathrm{E}-14$ & $2.68 \mathrm{E}-31$ & $2.93 \mathrm{E}+07$ & $1.43 E+04$ \\
\hline Eu-154 & $7.88 E-02$ & $9.85 E+03$ & $8.22 E+00$ & $1.18 E-06$ & $1.34 \mathrm{E}-30$ & $1.98 E-167$ & $0.00 E+00$ & $4.15 E+08$ & $5.65 E-26$ \\
\hline $\mathrm{U}-234$ & $2.83 E-06$ & $2.50 \mathrm{E}+00$ & $2.50 \mathrm{E}+00$ & $2.50 \mathrm{E}+00$ & $2.49 E+00$ & $2.46 \mathrm{E}+00$ & $2.43 E+00$ & $1.05 E+05$ & $1.05 E+05$ \\
\hline $\mathrm{U}-235$ & $9.76 \mathrm{E}-10$ & $3.63 E-02$ & $3.63 \mathrm{E}-02$ & $3.63 E-02$ & $3.63 E-02$ & $3.63 \mathrm{E}-02$ & $3.63 E-02$ & $1.53 E+03$ & $1.53 E+03$ \\
\hline $\mathrm{U}-236$ & $2.96 \mathrm{E}-08$ & -01 & $5.38 \mathrm{E}-01$ & $5.38 \mathrm{E}-01$ & $5.38 \mathrm{E}-01$ & $5.38 \mathrm{E}-01$ & $5.37 \mathrm{E}-01$ & $6 \mathrm{E}+04$ & $\overline{E+04}$ \\
\hline $\mathrm{U}-238$ & $1.55 \mathrm{E}-09$ & $6.66 \mathrm{E}-01$ & $6.66 \mathrm{E}-01$ & $6.66 \mathrm{E}-01$ & 6.66E-01 & $6.66 \mathrm{E}-01$ & $6.66 \mathrm{E}-01$ & $2.80 E+04$ & $2.80 E+04$ \\
\hline Th-230 & $9.00 \mathrm{E}-05$ & $0.00 E+00$ & $2.86 \mathrm{E}-02$ & $2.95 \mathrm{E}-01$ & $3.36 \mathrm{E}+00$ & $7.58 \mathrm{E}+01$ & $2.64 E+02$ & $0.00 E+00$ & $1.41 E+05$ \\
\hline$R a-226$ & 4.33E-04 & $0.00 E+00$ & $8.49 E-01$ & $2.77 E+01$ & $1.01 \mathrm{E}+03$ & $8.16 \mathrm{E}+04$ & $4.08 E+05$ & $0.00 E+00$ & $4.24 E+07$ \\
\hline Np-237 & $3.24 \mathrm{E}-07$ & $6.62 \mathrm{E}-01$ & $2.48 E+04$ & $8.28 E+04$ & $1.87 E+05$ & $2.36 \mathrm{E}+05$ & $2.36 E+05$ & $2.78 E+04$ & $7.85 \mathrm{E}+09$ \\
\hline $\mathrm{Pu}-238$ & $7.90 \mathrm{E}-03$ & $4.89 E+03$ & $2.40 E+03$ & $4.95 E+02$ & $1.96 \mathrm{E}+00$ & $3.71 \mathrm{E}-14$ & $2.60 \mathrm{E}-31$ & $2.06 E+08$ & $8.26 E+04$ \\
\hline $\mathrm{Pu}-239$ & $2.88 \mathrm{E}-05$ & $6.57 \mathrm{E}+02$ & $6.56 E+02$ & $6.52 E+02$ & $6.39 E+02$ & $5.69 \mathrm{E}+02$ & $4.93 E+02$ & $2.77 E+07$ & $2.69 E+07$ \\
\hline Pu-240 & $1.06 \mathrm{E}-04$ & $1.09 E+03$ & $1.08 E+03$ & $1.06 \mathrm{E}+03$ & $9.85 E+02$ & $6.46 \mathrm{E}+02$ & $3.81 E+02$ & $4.61 E+07$ & $4.15 E+07$ \\
\hline $\mathrm{Pu}-241$ & $4.81 E-02$ & $1.63 E+05$ & $2.14 E+03$ & $1.41 E-01$ & $3.28 E-16$ & $7.89 E-100$ & $2.36 \mathrm{E}-204$ & $6.86 \mathrm{E}+09$ & $1.38 \mathrm{E}-11$ \\
\hline Pu-242 & $1.86 \mathrm{E}-06$ & $3.61 \mathrm{E}+00$ & $3.61 \mathrm{E}+00$ & $3.61 E+00$ & $3.61 \mathrm{E}+00$ & $3.58 \mathrm{E}+00$ & $3.55 E+00$ & $1.52 E+05$ & $1.52 E+05$ \\
\hline$A m-241$ & $1.60 \mathrm{E}-03$ & $3.55 E+03$ & $1.51 \mathrm{E}+05$ & $1.08 E+05$ & $3.52 E+04$ & $5.81 E+01$ & $1.93 \mathrm{E}-02$ & $1.49 \mathrm{E}+08$ & $1.48 \mathrm{E}+09$ \\
\hline$A m-242 m$ & $4.92 \mathrm{E}-03$ & $1.46 E+01$ & $9.35 E+00$ & $3.50 \mathrm{E}+00$ & $1.12 \mathrm{E}-01$ & $3.23 \mathrm{E}-10$ & $6.83 \mathrm{E}-21$ & $6.13 E+05$ & $4.72 E+03$ \\
\hline Am-243 & $9.39 \mathrm{E}-05$ & $3.59 E+01$ & $3.56 E+01$ & $3.49 \mathrm{E}+01$ & $3.27 \mathrm{E}+01$ & $2.25 \mathrm{E}+01$ & $1.41 \mathrm{E}+01$ & $1.51 E+06$ & $1.38 E+06$ \\
\hline $\mathrm{Cm}-242$ & $1.55 \mathrm{E}+00$ & $1.20 E+01$ & $2.44 E-60$ & $3.30 E-195$ & $0.00 \mathrm{E}+00$ & $0.00 \mathrm{E}+00$ & $0.00 \mathrm{E}+00$ & $5.06 E+05$ & $0.00 E+00$ \\
\hline$c m-244$ & $3.83 \mathrm{E}-02$ & $2.77 E+03$ & $8.83 E+01$ & $4.17 \mathrm{E}-02$ & $9.50 \mathrm{E}-14$ & $2.83 E-80$ & $1.97 \mathrm{E}-163$ & $1.17 E+08$ & $4.00 E-09$ \\
\hline $\mathrm{Cm}-245$ & $8.15 \mathrm{E}-05$ & $3.07 E-01$ & $3.04 \mathrm{E}-01$ & 2.99E-01 & $2.83 E-01$ & $2.04 \mathrm{E}-01$ & $1.36 \mathrm{E}-01$ & $1.29 E+04$ & $1.19 E+04$ \\
\hline$c m-246$ & $1.47 \mathrm{E}-04$ & $6.55 \mathrm{E}-02$ & $6.47 \mathrm{E}-02$ & $6.28 \mathrm{E}-02$ & $5.67 \mathrm{E}-02$ & $3.15 \mathrm{E}-02$ & $1.52 \mathrm{E}-02$ & $2.76 \mathrm{E}+03$ & $2.39 E+03$ \\
\hline $\mathrm{Ba}-137 \mathrm{~m}$ & $1.50 \mathrm{E}+01$ & $1.63 E+05$ & $0.00 \mathrm{E}+00$ & $0.00 E+00$ & $0.00 E+00$ & $0.00 E+00$ & $0.00 E+00$ & $6.87 E+09$ & $0.00 E+00$ \\
\hline total & & $7.62 E+05$ & $2.18 \mathrm{E}+05$ & $1.94 E+05$ & $2.24 E+05$ & $3.19 E+05$ & $6.45 E+05$ & $3.21 E+10$ & $9.45 E+09$ \\
\hline
\end{tabular}


Table 4. Inventory of a LWR Spent Fuel Waste Package, 2 of 2

\begin{tabular}{|c|c|c|c|c|c|c|c|}
\hline Nuclide & Sp Activity & & g/pkg & at & year & & \\
\hline 42095 & $(C 1 / g)$ & 10 & 100 & 300 & 1000 & 5000 & 10000 \\
\hline$c-14$ & $4.46 \mathrm{E}+00$ & $7.30 \mathrm{E}-01$ & $7.22 E-01$ & $7.05 \mathrm{E}-01$ & \begin{tabular}{|l|}
$6.48 E-01$ \\
\end{tabular} & 3.99E-01 & $2.18 E-01$ \\
\hline $\mathrm{Ni}-59$ & $7.57 E+04$ & $1.43 E-04$ & $1.43 \mathrm{E}-04$ & $1.42 \mathrm{E}-04$ & $1.41 \mathrm{E}-04$ & $1.36 E-04$ & $1.30 \mathrm{E}-04$ \\
\hline Se-79 & $6.97 E-02$ & $1.24 E+01$ & $1.23 E+01$ & $1.23 E+01$ & $1.22 E+01$ & $1.17 \mathrm{E}+01$ & $1.11 E+01$ \\
\hline $5 x-90$ & $1.36 \mathrm{E}+02$ & $8.83 E+02$ & $9.90 \mathrm{E}+01$ & $7.64 \mathrm{E}-01$ & $3.08 E-08$ & $1.74 \mathrm{E}-50$ & $2.67 E-103$ \\
\hline$Y-90$ & $5.44 E+05$ & 2.21E-01 & $0.00 E+00$ & $0.00 \mathrm{E}+00$ & $0.00 \mathrm{E}+00$ & $0.00 \mathrm{E}+00$ & $0.00 \mathrm{E}+00$ \\
\hline $2 x-93$ & $2.51 \mathrm{E}-03$ & 1.61E+03 & $1.61 \mathrm{E}+03$ & $1.61 \mathrm{E}+03$ & $1.61 E+03$ & $1.61 E+03$ & $1.61 E+03$ \\
\hline $\mathrm{Nb}-94$ & 1.87E-01 & $1.44 E+01$ & $1.43 E+01$ & $1.42 E+01$ & $1.39 E+01$ & 1.21E+01 & $1.02 E+01$ \\
\hline TC-99 & $1.70 \mathrm{E}-02$ & $1.62 E+03$ & $1.62 \mathrm{E}+03$ & $1.62 \mathrm{E}+03$ & $1.62 \mathrm{E}+03$ & $1.60 \mathrm{E}+03$ & $1.57 E+03$ \\
\hline Pd-107 & 5.14E-04 & $4.58 E+02$ & $4.58 E+02$ & $4.58 E+02$ & $4.58 E+02$ & $4.57 E+02$ & $4.57 E+02$ \\
\hline $5 n-126$ & $2.84 E-02$ & $5.74 E+01$ & $5.73 E+01$ & $5.73 E+01$ & $5.70 \mathrm{E}+01$ & $5.54 E+01$ & $5.35 E+01$ \\
\hline$x-129$ & $1.77 \mathrm{E}-04$ & $3.74 E+02$ & $3.74 \mathrm{E}+02$ & $3.74 E+02$ & $3.74 E+02$ & $3.74 E+02$ & $3.74 E+02$ \\
\hline Cs-135 & $1.15 \mathrm{E}-03$ & $6.30 \mathrm{E}+02$ & $6.30 E+02$ & $6.30 \mathrm{E}+02$ & $6.30 E+02$ & $6.29 E+02$ & $3 E+02$ \\
\hline Cs-137 & $8.70 \mathrm{E}+01$ & $1.98 E+03$ & $2.48 E+02$ & $2.44 E+00$ & 2.31E-07 & $1.68 \mathrm{E}-47$ & $1.13 E-97$ \\
\hline sm-151 & $2.63 E+01$ & $2.64 E+01$ & $1.32 \mathrm{E}+01$ & $2.83 E+00$ & 1.29E-02 & $5.39 E-16$ & $2 E-32$ \\
\hline Eu-154 & $2.70 E+02$ & $3.65 E+01$ & 3.04E-02 & 4.38E-09 & $4.97 \mathrm{E}-33$ & $7.32 \mathrm{E}-170$ & $0.00 \mathrm{E}+00$ \\
\hline $\mathrm{U}-234$ & $6.25 E-03$ & $4.00 E+02$ & $4.00 \mathrm{E}+02$ & $4.00 E+02$ & $3.99 E+02$ & $3.94 \mathrm{E}+02$ & $3.89 E+02$ \\
\hline$U-235$ & $2.16 \mathrm{E}-06$ & $1.68 E+04$ & $1.68 E+04$ & $1.68 E+04$ & $1.68 E+04$ & $1.68 \mathrm{E}+04$ & $8 E+04$ \\
\hline $\mathrm{U}-236$ & 6.47E-05 & $8.31 E+03$ & $8.31 E+03$ & $8.31 E+03$ & $8.31 E+03$ & $8.31 E+03$ & $8.31 E+03$ \\
\hline $\mathrm{U}-238$ & 3.36E-07 & $1.98 E+06$ & $1.98 E+06$ & $1.98 E+06$ & $1.98 E+06$ & $1.98 E+06$ & $1.98 E+06$ \\
\hline Th-230 & 2.02E-01 & $0.00 E+00$ & $1.41 E-01$ & $1.46 \mathrm{E}+00$ & $1.66 \mathrm{E}+01$ & $3.75 E+02$ & $1.31 E+03$ \\
\hline $\mathrm{Ra}-226$ & $9.88 \mathrm{E}-01$ & $0.00 E+00$ & $8.59 E-01$ & $2.80 \mathrm{E}+01$ & $1.02 E+03$ & $8.26 \mathrm{E}+04$ & 4.13E+05 \\
\hline$N p-237$ & $7.05 E-04$ & $9.38 \mathrm{E}+02$ & $3.52 E+07$ & $1.17 E+08$ & $2.65 E+08$ & $3.35 E+08$ & $3.35 E+08$ \\
\hline $\mathrm{Pu}-238$ & $1.71 E+01$ & $2.86 E+02$ & $1.40 \mathrm{E}+02$ & $2.89 E+01$ & $1.15 E-01$ & $2.17 \mathrm{E}-15$ & $1.52 E-32$ \\
\hline $\mathrm{Qu}-239$ & $6.22 \mathrm{E}-02$ & $1.06 E+04$ & $1.05 E+04$ & $1.05 E+04$ & $1.03 E+04$ & $9.15 \mathrm{E}+03$ & $7.93 E+03$ \\
\hline Pu-240 & $2.28 \mathrm{E}-01$ & $4.80 E+03$ & $4.75 E+03$ & $4.65 E+03$ & $4.32 \mathrm{E}+03$ & $2.83 E+03$ & $1.67 E+03$ \\
\hline $\mathrm{Pu}-241$ & $1.03 E+02$ & $1.58 E+03$ & $2.08 E+01$ & 1.37E-03 & $3.19 \mathrm{E}-18$ & $7.66 \mathrm{E}-102$ & $2.29 E-206$ \\
\hline Pu-242 & $3.82 E-03$ & $9.46 E+02$ & $9.45 E+02$ & $9.45 \mathrm{E}+02$ & $9.44 E+02$ & $9.37 \mathrm{E}+02$ & $9.28 \mathrm{E}+02$ \\
\hline$A m-241$ & $3.43 E+00$ & $1.03 E+03$ & $4.41 E+04$ & $3.15 E+04$ & $1.03 E+04$ & $1.69 E+01$ & 5.63E-03 \\
\hline$A m-242 m$ & $9.72 E+00$ & $1.50 E+00$ & 9.62E-01 & $3.60 \mathrm{E}-01$ & $1.15 \mathrm{E}-02$ & $3.33 \mathrm{E}-11$ & $7.03 E-22$ \\
\hline Am-243 & $1.99 \mathrm{E}-01$ & $1.80 \mathrm{E}+02$ & $1.79 E+02$ & $1.76 \mathrm{E}+02$ & $1.64 E+02$ & $1.13 E+02$ & $7.06 \mathrm{E}+01$ \\
\hline$C m-242$ & $3.31 E+03$ & $3.63 E-03$ & $7.38 \mathrm{E}-64$ & $9.96 \mathrm{E}-199$ & $0.00 E+00$ & $0.00 \mathrm{E}+00$ & $0.00 \mathrm{E}+00$ \\
\hline $\mathrm{Cm}-244$ & $8.09 E+01$ & $3.43 E+01$ & $1.09 \mathrm{E}+00$ & $5.15 \mathrm{E}-04$ & $1.17 E-15$ & $3.50 \mathrm{E}-82$ & $2.43 E-165$ \\
\hline$c m-245$ & $1.72 \mathrm{E}-01$ & $1.78 E+00$ & $1.77 \mathrm{E}+00$ & $1.74 E+00$ & $1.64 E+00$ & $1.19 \mathrm{E}+00$ & 7.89E-01 \\
\hline $\mathrm{C} m-246$ & $3.07 \mathrm{E}-01$ & $2.13 E-01$ & $2.11 E-01$ & 2.05E-01 & $1.85 E-01$ & $1.03 E-01$ & $4.94 \mathrm{E}-02$ \\
\hline
\end{tabular}


Table 5. Inventory of and A1-1,2 Package, 1 of 2

\begin{tabular}{|c|c|c|c|c|c|c|c|c|c|}
\hline Nuclide & Lambda & & Ci/pkg & at & year & & & stream & total \\
\hline 4190 & $(1 a)$ & 10 & 100 & 300 & 1000 & 5000 & 10000 & $\mathrm{Cl}$ at $10 \mathrm{a}$ & $\mathrm{Ci}$ at 1000 a \\
\hline$c-14$ & $1.21 E-04$ & $2.39 E+01$ & $2.36 E+01$ & $2.31 E+01$ & $2.12 E+01$ & 1.31E+01 & $7.14 E+00$ & $1.00 \mathrm{E}+05$ & $8.88 E+04$ \\
\hline $\mathrm{Ni}-59$ & $9.24 \mathrm{E}-06$ & $9.11 E+01$ & $9.10 E+01$ & $9.08 E+01$ & $9.02 \mathrm{E}+01$ & $8.70 E+01$ & $8.30 E+01$ & $3.82 E+05$ & $3.78 E+05$ \\
\hline $5 e-79$ & $1.07 E-05$ & & & & & & & & \\
\hline $5 x-90$ & $2.43 E-02$ & & & & & & & & \\
\hline$Y-90$ & $9.48 \mathrm{E}+01$ & & & & & & & & \\
\hline $\mathrm{zr}-93$ & 4.53E-07 & $1.99 E+00$ & $1.99 E+00$ & $1.99 E+00$ & $1.99 E+00$ & $1.99 \mathrm{E}+00$ & $1.98 \mathrm{E}+00$ & $8.34 E+03$ & $8.33 E+03$ \\
\hline $\mathrm{Nb}-94$ & $3.41 \mathrm{E}-05$ & $1.91 E+01$ & $1.91 E+01$ & $1.89 E+01$ & $1.85 E+01$ & $1.61 E+01$ & $1.36 E+01$ & $8.01 E+04$ & $7.74 E+04$ \\
\hline TC-99 & $3.29 \mathrm{E}-06$ & $1.60 E-02$ & $1.60 \mathrm{E}-02$ & $1.60 \mathrm{E}-02$ & $1.59 \mathrm{E}-02$ & 1.57E-02 & $1.55 \mathrm{E}-02$ & $6.70 E+01$ & $6.68 E+01$ \\
\hline $\mathrm{Pd}-107$ & $1.07 E-07$ & & & & & & & & \\
\hline $5 n-126$ & 6.93E-06 & & & & & & & & \\
\hline$I-129$ & $4.41 \mathrm{E}-08$ & & & & & & & & \\
\hline $\mathrm{Cs}-135$ & $3.01 \mathrm{E}-07$ & & & & & & & & \\
\hline Cs-137 & $2.31 E-02$ & & & & & & & & \\
\hline $\mathrm{U}-234$ & $2.83 E-06$ & $1.72 \mathrm{E}-02$ & $1.72 \mathrm{E}-02$ & 1.72E-02 & $1.71 \mathrm{E}-02$ & $1.70 \mathrm{E}-02$ & 1.67E-02 & $7.20 \mathrm{E}+01$ & $7.18 E+01$ \\
\hline $\mathrm{U}-235$ & $9.76 \mathrm{E}-10$ & $2.95 \mathrm{E}-04$ & $2.95 \mathrm{E}-04$ & $2.95 \mathrm{E}-04$ & 2.95E-04 & $2.95 E-04$ & 2.95E-04 & $1.23 E+00$ & $1.23 E+00$ \\
\hline $\mathrm{U}-236$ & $2.96 \mathrm{E}-08$ & $3.81 \mathrm{E}-03$ & $3.81 \mathrm{E}-03$ & $3.81 E-03$ & $3.81 \mathrm{E}-03$ & $3.81 \mathrm{E}-03$ & $3.81 \mathrm{E}-03$ & $1.59 E+01$ & $1.59 E+01$ \\
\hline $\mathrm{U}-238$ & $1.55 \mathrm{E}-09$ & $4.67 E-03$ & $4.67 \mathrm{E}-03$ & $4.67 \mathrm{E}-03$ & \begin{tabular}{|l|}
$4.67 \mathrm{E}-03$ \\
\end{tabular} & 4.67E-03 & 4.67E-03 & $1.95 E+01$ & $1.95 E+01$ \\
\hline Th-230 & $9.00 \mathrm{E}-05$ & & $1.96 \mathrm{E}-04$ & $2.03 E-03$ & $2.31 \mathrm{E}-02$ & 5.22E-01 & $1.82 E+00$ & $0.00 E+00$ & $9.69 E+01$ \\
\hline Ra-226 & $4.33 E-04$ & & $5.84 \mathrm{E}-03$ & $1.90 \mathrm{E}-01$ & $6.93 \mathrm{E}+00$ & $5.61 \mathrm{E}+02$ & $2.81 E+03$ & $0.00 E+00$ & $2.90 E+04$ \\
\hline$N_{p}-237$ & $3.24 E-07$ & 4.67E-03 & 1.77E+02 & $5.92 E+02$ & $1.33 E+03$ & $1.69 E+03$ & $1.69 E+03$ & $1.95 E+01$ & $5.58 E+06$ \\
\hline$P u-238$ & $7.90 \mathrm{E}-03$ & $3.32 E+01$ & $1.63 E+01$ & $3.35 E+00$ & \begin{tabular}{|l|}
$1.33 E-02$ \\
\end{tabular} & $2.51 \mathrm{E}-16$ & $1.76 \mathrm{E}-33$ & $1.39 E+05$ & $5.57 E+01$ \\
\hline Pu-239 & $2.88 \mathrm{E}-05$ & $4.67 E+00$ & $4.65 E+00$ & $4.63 E+00$ & $4.53 E+00$ & $4.04 E+00$ & $3.50 \mathrm{E}+00$ & $1.95 E+04$ & $1.90 E+04$ \\
\hline $\mathrm{Pu}-240$ & $1.06 \mathrm{E}-04$ & $7.86 \mathrm{E}+00$ & $7.78 E+00$ & $7.62 E+00$ & $7.08 \mathrm{E}+00$ & $4.64 \mathrm{E}+00$ & $2.74 \mathrm{E}+00$ & $3.29 E+04$ & $2.97 E+04$ \\
\hline Pu-241 & $4.81 \mathrm{E}-02$ & $1.13 E+03$ & $1.48 \mathrm{E}+01$ & $9.78 \mathrm{E}-04$ & $2.28 \mathrm{E}-18$ & $5.47 \mathrm{E}-102$ & 1.63E-206 & $4.73 E+06$ & $9.54 E-15$ \\
\hline Pu-242 & 1.86E-06 & $2.58 \mathrm{E}-02$ & $2.58 \mathrm{E}-02$ & $2.58 \mathrm{E}-02$ & 2.57E-02 & $2.55 \mathrm{E}-02$ & 2.53E-02 & $1.08 E+02$ & $1.08 E+02$ \\
\hline Am-241 & $1.60 \mathrm{E}-03$ & $2.70 E+01$ & $1.05 E+03$ & $7.51 E+02$ & $2.45 E+02$ & $4.04 E-01$ & 1.34E-04 & $1.13 E+05$ & $1.03 E+06$ \\
\hline Am-242m & $4.92 \mathrm{E}-03$ & 8.84E-02 & 5.68E-02 & $2.12 \mathrm{E}-02$ & $6.81 \mathrm{E}-04$ & $1.96 \mathrm{E}-12$ & $4.15 E-23$ & $3.70 \mathrm{E}+02$ & $2.85 E+00$ \\
\hline $\mathrm{Am}-243$ & $9.39 \mathrm{E}-05$ & $2.58 \mathrm{E}-01$ & $2.56 \mathrm{E}-01$ & $2.51 E-01$ & $2.35 \mathrm{E}-01$ & $1.61 \mathrm{E}-01$ & 1.01E-01 & $1.08 E+03$ & $9.84 E+02$ \\
\hline $\mathrm{Cm}-242$ & $1.55 \mathrm{E}+00$ & $7.24 \mathrm{E}-02$ & 1.47E-62 & $1.99 \mathrm{E}-197$ & $0.00 \mathrm{E}+00$ & $0.00 \mathrm{E}+00$ & $0.00 \mathrm{E}+00$ & $3.04 E+02$ & $0.00 E+00$ \\
\hline$C m-244$ & 3.83E-02 & $1.96 \mathrm{E}+01$ & $6.26 \mathrm{E}-01$ & $2.95 \mathrm{E}-04$ & $6.73 \mathrm{E}-16$ & $2.01 E-82$ & $1.40 \mathrm{E}-165$ & $8.23 E+04$ & $2.82 \mathrm{E}-12$ \\
\hline $\mathrm{Cm}-245$ & $8.15 E-05$ & $2.21 \mathrm{E}-03$ & $2.19 \mathrm{E}-03$ & $2.16 \mathrm{E}-03$ & $2.04 \mathrm{E}-03$ & $1.47 \mathrm{E}-03$ & $9.79 \mathrm{E}-04$ & $9.26 \mathrm{E}+00$ & $8.54 E+00$ \\
\hline $\mathrm{cm}-246$ & $1.47 \mathrm{E}-04$ & $4.54 E-04$ & $4.48 E-04$ & 4.35E-04 & $3.93 \mathrm{E}-04$ & 2.19E-04 & 1.05E-04 & $1.90 \mathrm{E}+00$ & $1.65 \mathrm{E}+00$ \\
\hline other act & & $9.70 E-01$ & $9.70 \mathrm{E}-01$ & $9.70 \mathrm{E}-01$ & 9.70E-01 & $9.70 \mathrm{E}-01$ & $9.70 E-01$ & $4.06 \mathrm{E}+03$ & \\
\hline others & & $6.21 E+04$ & & & & & & $2.60 E+08$ & \\
\hline lotal & & $6.35 E+04$ & $1.41 E+03$ & $1.49 E+03$ & $1.73 E+03$ & $2.38 \mathrm{E}+03$ & $4.61 E+03$ & $2.66 \mathrm{E}+08$ & \\
\hline
\end{tabular}




\begin{tabular}{|c|c|c|c|c|c|c|c|}
\hline Nuclide & Sp Activity & & g/pkg & at & year & & \\
\hline 4190 & $(\mathrm{Cl} / \mathrm{g})$ & 10 & 100 & 300 & 1000 & 5000 & 10000 \\
\hline$c-14$ & $4.46 \mathrm{E}+00$ & $5.36 \mathrm{E}+00$ & $5.30 \mathrm{E}+00$ & $5.18 \mathrm{E}+00$ & $4.76 E+00$ & $2.93 E+00$ & $1.60 \mathrm{E}+00$ \\
\hline $\mathrm{Ni}-59$ & $7.57 \mathrm{E}+04$ & $1.20 \mathrm{E}-03$ & $1.20 \mathrm{E}-03$ & $1.20 \mathrm{E}-03$ & $1.19 \mathrm{E}-03$ & & \\
\hline Sè-79 & $6.97 \mathrm{E}-02$ & $0.00 \mathrm{E}+00$ & $0.00 E+00$ & $0.00 \mathrm{E}+00$ & $0.00 E+00$ & $0.00 \mathrm{E}$ & \\
\hline$s r-90$ & $1.36 \mathrm{E}+02$ & $0.00 E+00$ & $0.00 \mathrm{E}+00$ & $0.00 \mathrm{E}+00$ & $0.00 E+00$ & $0.00 E+00$ & $0 \mathrm{E}+00$ \\
\hline$Y-90$ & $5.44 E+05$ & $0.00 E+00$ & $0.00 E+00$ & $0.00 E+00$ & $0.00 E+00$ & $0.00 E+00$ & $00 \mathrm{E}+00$ \\
\hline$z x-93$ & 2.51 & $7.93 \mathrm{E}+02$ & $7.93 E+02$ & $7.93 E+02$ & $7.92 \mathrm{E}+02$ & $7.91 E+02$ & $.89 E+02$ \\
\hline $\mathrm{Nb}-94$ & $1.87 \mathrm{E}-01$ & $1.02 E+02$ & $1.02 E+02$ & $1.01 \mathrm{E}+02$ & $9.87 E+01$ & $8.61 \mathrm{E}+01$ & $7.26 \mathrm{E}+01$ \\
\hline Tc-99 & $1.70 \mathrm{E}-02$ & $9.44 \mathrm{E}-01$ & $9.44 \mathrm{E}-01$ & $9.43 \mathrm{E}-01$ & $9.41 \mathrm{E}-01$ & $9.29 \mathrm{E}-01$ & $3 E-01$ \\
\hline$P d-107$ & -04 & 0.00 & $0.00 E+00$ & $0.00 E+00$ & $0.00 E+00$ & $0.00 E+00$ & $0.00 \mathrm{E}+00$ \\
\hline Sn-126 & $=-02$ & $0.00 E+00$ & $0.00 E+00$ & $0.00 E+00$ & $0.00 E+00$ & +00 & $E+00$ \\
\hline$I-129$ & -04 & +00 & $0.00 E+00$ & $E+00$ & $E+00$ & +00 & $E+00$ \\
\hline $\mathrm{Cs}-$ & & & +00 & $E+00$ & $E+00$ & +00 & $E+00$ \\
\hline Cs- & & & & & +00 & +00 & $E+00$ \\
\hline$U-234$ & 3 & 2.7 & 2.75 & +00 & & & \\
\hline $\mathrm{u}-235$ & 6 & 1.3 & 1.3 & $E+02$ & $E+02$ & +02 & $E+02$ \\
\hline U-236 & 05 & $5.88 \mathrm{E}+01$ & 5.88 & $5.88 E+01$ & $5.88 E+01$ & +01 & $E+01$ \\
\hline$u-238$ & .07 & $1.39 E+04$ & $1.39 E+04$ & $1.39 E+04$ & $1.39 E+04$ & +04 & $1.39 E+04$ \\
\hline$T h-230$ & 01 & $0.00 E+00$ & $9.72 E-04$ & $1.00 E-02$ & $1.14 \mathrm{E}-01$ & $2.58 E+00$ & $9.00 E+00$ \\
\hline Ra-226 & 01 & $0.00 E+00$ & $5.91 \mathrm{E}-03$ & 1.93E-01 & $7.01 E+00$ & $5.68 \mathrm{E}+02$ & $2.84 E+03$ \\
\hline Np-237 & -04 & $6.62 \mathrm{E}+00$ & $2.52 E+05$ & $8.39 E+05$ & $1.89 E+06$ & $2.39 E+06$ & $2.39 E+06$ \\
\hline $\mathrm{Pu}-238$ & 01 & +00 & 9.51 & $1.96 E-01$ & 7.77E-04 & $1.47 \mathrm{E}-17$ & $3 E-34$ \\
\hline Pu-239 & & & & +01 & +01 & +01 & +01 \\
\hline Pu- & & & & & 01 & & 01 \\
\hline & & 01 & 1.4 & & -20 & & 208 \\
\hline $\mathrm{Pu}-242$ & -03 & 6.7 & 6.7 & +00 & $E+00$ & .00 & +00 \\
\hline$A m-241$ & +00 & $7.88 E+00$ & $3.06 E+02$ & +02 & 7.1 & & -05 \\
\hline$A m-242 m$ & $E+00$ & $9.10 \mathrm{E}-03$ & 5.84 & 03 & 7.0 & -13 & -24 \\
\hline Am-243 & 9E-01 & $1.30 E+00$ & $1.28 E+00$ & +00 & $=+00$ & -01 & -01 \\
\hline$c m-242$ & $E+03$ & $2.19 E-05$ & $4.45 E-66$ & $6.01 \mathrm{E}-201$ & $0.00 E+00$ & $0.00 \mathrm{E}+00$ & +00 \\
\hline $\mathrm{Cm}-244$ & +01 & 2.43 & $7.74 \mathrm{E}-03$ & -06 & $=-18$ & -84 & 167 \\
\hline 45 & & & 1.2 & & -02 & & $=-03$ \\
\hline $\mathrm{Cm}-246$ & $3.07 \mathrm{E}-01$ & $1.48 \mathrm{E}-03$ & $1.46 \mathrm{E}-03$ & $1.42 E-03$ & $1.28 \mathrm{E}-03$ & $7.12 \mathrm{E}-04$ & 3.42E-04 \\
\hline
\end{tabular}


Table 6. Inventory of an A1-3 Package

\begin{tabular}{|c|c|c|c|c|c|c|c|}
\hline Nuclide & Lambda & & & Ci/pkg at $\mathrm{yr}$ & & & \\
\hline 5 & $(/ \mathrm{a})$ & 10 & 100 & 300 & 1000 & 5000 & 10000 \\
\hline I-129 & $4.41 \mathrm{E}-08$ & $3.97 \mathrm{E}+02$ & $3.97 \mathrm{E}+02$ & $3.97 \mathrm{E}+02$ & $3.97 \mathrm{E}+02$ & $3.97 \mathrm{E}+02$ & $3.97 \mathrm{E}+02$ \\
\hline TOTAL & & $3.97 \mathrm{E}+02$ & $3.97 \mathrm{E}+02$ & $3.97 \mathrm{E}+02$ & $3.97 \mathrm{E}+02$ & $3.97 \mathrm{E}+02$ & $3.97 \mathrm{E}+02$ \\
\hline & & & & & & & \\
\hline Nuclide & Sp Activity & & & $\mathrm{g} / \mathrm{pkg}$ at $\mathrm{yr}$ & & & stream total \\
\hline 5 & $($ Ci/g) & 10 & 100 & 300 & 1000 & & Ci at 10 a \\
\hline $\mathrm{I}-129$ & $1.77 \mathrm{E}-04$ & $2.24 \mathrm{E}+06$ & $2.24 \mathrm{E}+06$ & $2.24 \mathrm{E}+06$ & $2.24 \mathrm{E}+06$ & & $1.98 \mathrm{E}+03$ \\
\hline
\end{tabular}


Table 7. Inventory of an A1-4 Package

\begin{tabular}{|c|c|c|c|c|c|c|c|c|c|}
\hline Nuclide & Lambda & & & Ci/pkg & at & year & & stream & total \\
\hline 25589 & $(/ a)$ & 10 & 100 & 300 & 1000 & 5000 & 10000 & Ci at 10 a & Ci at 1000a \\
\hline $5 x-90$ & $2.43 \mathrm{E}-02$ & $1.38 E+05$ & $1.55 E+04$ & $1.20 E+02$ & $4.83 E-06$ & $2.72 E-48$ & $4.19 E-101$ & $3.54 E+09$ & $1.24 \mathrm{E}-01$ \\
\hline$Y-90$ & $9.48 \mathrm{E}+01$ & $1.38 E+05$ & $0.00 E+00$ & $0.00 E+00$ & $0.00 E+00$ & $0.00 E+00$ & $0.00 E+00$ & $3.54 E+09$ & $0.00 E+00$ \\
\hline Cs-135 & $3.01 \mathrm{E}-07$ & $8.40 E-01$ & $8.40 E-01$ & $8.40 E-01$ & $8.40 E-01$ & $8.39 E-01$ & $8.37 \mathrm{E}-01$ & $2.15 E+04$ & $2.15 E+04$ \\
\hline Cs-137 & $2.31 \mathrm{E}-02$ & $1.99 E+05$ & $2.48 E+04$ & $2.45 E+02$ & 2.31E-05 & $1.69 E-45$ & $1.14 \mathrm{E}-95$ & $5.09 E+09$ & $5.92 E-01$ \\
\hline others & & $2.68 E+05$ & $0.00 \mathrm{E}+00$ & $0.00 E+00$ & $0.00 E+00$ & $0.00 E+00$ & $0.00 \mathrm{E}+00$ & $6.85 E+09$ & $0.00 E+00$ \\
\hline TOTAL & & $7.43 E+05$ & $4.03 E+04$ & $3.65 E+02$ & $8.40 E-01$ & $8.39 E-01$ & $8.37 E-01$ & $1.90 E+10$ & $2.15 E+04$ \\
\hline & & & & & & & & & \\
\hline Nuclide & Sp Activity & & & q/pkq & at & year & & & \\
\hline & $(\mathrm{Ci} / \mathrm{g})$ & 10 & 100 & 300 & 1000 & 5000 & 00 & & \\
\hline Sr-90 & $1.36 \mathrm{E}+02$ & $1.02 E+03$ & $1.14 \mathrm{E}+02$ & $8.80 E-01$ & $3.55 \mathrm{E}-08$ & $2.00 E-50$ & $3.08 \mathrm{E}-103$ & & \\
\hline$Y-90$ & $5.44 E+05$ & $2.54 \mathrm{E}-01$ & $0.00 E+00$ & $0.00 E+00$ & $0.00 E+00$ & $0.00 E+00$ & $0.00 E+00$ & & \\
\hline$C s-135$ & $1.15 \mathrm{E}-03$ & $7.30 E+02$ & $7.30 E+02$ & $7.30 \mathrm{E}+02$ & $7.30 E+02$ & $7.29 E+02$ & $7.28 E+02$ & & \\
\hline Cs-137 & $8.70 \mathrm{E}+01$ & $2.28 E+03$ & $2.86 E+02$ & $2.81 E+00$ & $2.66 \mathrm{E}-07$ & $1.94 \mathrm{E}-47$ & $1.31 E-97$ & & \\
\hline
\end{tabular}


Table 8. Inventory of an A1-6 Package

\begin{tabular}{|c|c|c|c|c|c|c|c|c|c|}
\hline Nuclide & Lambda & & Ci/pkg & at & year & & & stream & total \\
\hline 2394 & $(/ a)$ & 10 & 100 & 300 & 1000 & 5000 & 10000 & Ci at 10 a & $\mathrm{Cl}$ at $1000 \mathrm{a}$ \\
\hline $\mathrm{Se}-79$ & $1.07 E-05$ & $1.07 \mathrm{E}+01$ & $1.07 E+01$ & $1.07 E+01$ & $1.06 \mathrm{E}+01$ & $1.02 E+01$ & $9.65 E+00$ & $2.57 E+04$ & $2.54 E+04$ \\
\hline $2 x-93$ & $4.53 E-07$ & $4.74 E+01$ & $4.74 E+01$ & $4.74 E+01$ & $4.74 E+01$ & $4.73 E+01$ & $4.72 E+01$ & $1.13 E+05$ & $1.13 E+05$ \\
\hline TC-99 & $3.29 E-06$ & $3.43 E+02$ & $3.43 E+02$ & $3.43 E+02$ & $3.42 \mathrm{E}+02$ & $3.37 E+02$ & $3.32 E+02$ & $8.21 E+05$ & $8.18 E+05$ \\
\hline Pd-107 & 1.07E-07 & $2.95 E+00$ & $2.95 E+00$ & $2.95 E+00$ & $2.95 E+00$ & $2.95 E+00$ & $2.95 E+00$ & $7.06 E+03$ & $7.06 E+03$ \\
\hline$S n-126$ & $6.93 E-06$ & $2.04 E+01$ & $2.04 E+01$ & $2.04 E+01$ & $2.03 E+01$ & $1.97 E+01$ & $1.90 E+01$ & $4.88 E+04$ & $4.85 E+04$ \\
\hline $\mathrm{U}-234$ & $2.83 E-06$ & $7.22 \mathrm{E}-01$ & $7.22 \mathrm{E}-01$ & $7.21 \mathrm{E}-01$ & $7.20 \mathrm{E}-01$ & $7.12 E-01$ & $7.02 E-01$ & $1.73 E+03$ & $1.72 E+03$ \\
\hline $\mathrm{U}-235$ & $9.76 \mathrm{E}-10$ & $1.24 \mathrm{E}-02$ & 1.24E-02 & $1.24 \mathrm{E}-02$ & $1.24 \mathrm{E}-02$ & $1.24 \mathrm{E}-02$ & $1.24 \mathrm{E}-02$ & $2.96 \mathrm{E}+01$ & $2.96 E+01$ \\
\hline $\mathrm{U}-236$ & $2.96 \mathrm{E}-08$ & $1.60 \mathrm{E}-01$ & $1.60 \mathrm{E}-01$ & $1.60 \mathrm{E}-01$ & $1.60 \mathrm{E}-01$ & $1.60 \mathrm{E}-01$ & $1.60 \mathrm{E}-01$ & $3.83 E+02$ & $3.83 E+02$ \\
\hline$U-238$ & $1.55 \mathrm{E}-09$ & $1.96 \mathrm{E}-01$ & 1.96E-01 & $1.96 \mathrm{E}-01$ & $1.96 \mathrm{E}-01$ & $1.96 \mathrm{E}-01$ & $1.96 \mathrm{E}-01$ & $4.69 \mathrm{E}+02$ & $4.69 \mathrm{E}+02$ \\
\hline Th-230 & $9.00 \mathrm{E}-05$ & $0.00 \mathrm{E}+00$ & $8.25 E-03$ & $8.51 \mathrm{E}-02$ & $9.71 \mathrm{E}-01$ & $2.19 E+01$ & $7.64 \mathrm{E}+01$ & $0.00 E+00$ & $2.32 E+03$ \\
\hline Ra-226 & $4.33 E-04$ & $0.00 E+00$ & $2.45 \mathrm{E}-01$ & $7.99 E+00$ & $2.91 \mathrm{E}+02$ & $2.36 \mathrm{E}+04$ & $1.18 E+05$ & $0.00 E+00$ & $6.96 \mathrm{E}+05$ \\
\hline others & & $5.63 \mathrm{E}+04$ & & & & & & $1.35 E+08$ & 0 \\
\hline total & & $5.67 \mathrm{E}+04$ & $4.26 \mathrm{E}+02$ & $4.33 E+02$ & $7.16 \mathrm{E}+02$ & $2.40 E+04$ & $1.18 \mathrm{E}+05$ & $1.36 \mathrm{E}+08$ & $1.71 E+06$ \\
\hline & & & & & & & & & \\
\hline & & $\therefore$ & & & & & & & \\
\hline Nuclide & Sp Activity & & g/pkg & at & year & & & & \\
\hline 2394 & $(\mathrm{Cl} / \mathrm{g})$ & 10 & 100 & 300 & 1000 & 5000 & 10000 & & \\
\hline $\mathrm{Se}-79$ & $6.97 \mathrm{E}-02$ & $1.54 E+02$ & $1.54 \mathrm{E}+02$ & $1.54 E+02$ & $1.53 E+02$ & $1.46 E+02$ & $1.39 E+02$ & & \\
\hline $2 x-93$ & $2.51 E-03$ & $1.89 E+04$ & $1.89 E+04$ & $1.89 E+04$ & $1.89 E+04$ & $1.88 E+04$ & $1.88 E+04$ & & \\
\hline TC-99 & $1.70 E-02$ & $2.02 E+04$ & $2.02 E+04$ & $2.02 E+04$ & $2.02 E+04$ & $1.99 E+04$ & $1.96 \mathrm{E}+04$ & & \\
\hline Pd-107 & $5.14 \mathrm{E}-04$ & $5.74 \mathrm{E}+03$ & $5.74 E+03$ & $5.74 \mathrm{E}+03$ & $5.74 E+03$ & $5.74 E+03$ & $5.73 E+03$ & & \\
\hline$s n-126$ & $2.84 \mathrm{E}-02$ & $7.18 \mathrm{E}+02$ & $7.18 E+02$ & $7.17 E+02$ & $7.13 E+02$ & $6.94 \mathrm{E}+02$ & $6.70 E+02$ & & \\
\hline $\mathrm{U}-234$ & $6.25 \mathrm{E}-03$ & $1.15 E+02$ & $1.16 \mathrm{E}+02$ & $1.15 \mathrm{E}+02$ & $1.15 E+02$ & $1.14 E+02$ & $1.12 E+02$ & & \\
\hline $\mathrm{U}-235$ & $2.16 \mathrm{E}-06$ & $5.73 E+03$ & $5.73 E+03$ & $5.73 E+03$ & $5.73 \mathrm{E}+03$ & $5.73 E+03$ & $5.73 E+03$ & & \\
\hline $\mathrm{U}-236$ & $6.47 E-05$ & $2.47 \mathrm{E}+03$ & $2.47 E+03$ & $2.47 E+03$ & $2.47 \mathrm{E}+03$ & $2.47 E+03$ & $2.47 E+03$ & & \\
\hline $\mathrm{U}-238$ & $3.36 \mathrm{E}-07$ & $5.83 E+05$ & $5.83 E+05$ & $5.83 E+05$ & $5.83 E+05$ & $5.83 E+05$ & $5.83 E+05$ & & \\
\hline$T h-230$ & $2.02 E-01$ & $0.00 E+00$ & $4.08 E-02$ & $4.21 E-01$ & $4.81 E+00$ & $1.08 E+02$ & $3.78 E+02$ & & \\
\hline $\mathrm{Ra}-226$ & $9.88 \mathrm{E}-01$ & $0.00 E+00$ & $2.48 E-01$ & $8.09 E+00$ & $2.94 E+02$ & $2.38 E+04$ & $1.19 E+05$ & & \\
\hline
\end{tabular}


Table 9. Inventory of an A1-7 Package

\begin{tabular}{|c|c|c|c|c|c|c|c|c|}
\hline Nuclide & Lambda & & & $\mathrm{Ci} / \mathrm{pkg}$ & at & year & & stream \\
\hline 1646 & $(/ a)$ & 10 & 100 & 300 & 1000 & 5000 & 10000 & $\mathrm{Ci}$ at $10 \mathrm{a}$ \\
\hline $5 x-90$ & $2.43 E-02$ & $2.18 E+04$ & $2.44 E+03$ & $1.89 \mathrm{E}+01$ & 7.61E-07 & $4.28 E-49$ & $6.60 \mathrm{E}-102$ & $3.59 E+07$ \\
\hline$Y-90$ & $9.48 \mathrm{E}+01$ & $2.18 \mathrm{E}+04$ & $0.00 E+00$ & $0.00 \mathrm{E}+00$ & $0.00 E+00$ & $0.00 \mathrm{E}+00$ & $0.00 \mathrm{E}+00$ & $3.59 \mathrm{E}+07$ \\
\hline Cs-135 & $3.01 \mathrm{E}-07$ & $1.40 \mathrm{E}-01$ & $1.40 \mathrm{E}-01$ & $1.40 E-01$ & $1.40 \mathrm{E}-01$ & $1.40 \mathrm{E}-01$ & $1.40 \mathrm{E}-01$ & $2.30 \mathrm{E}+02$ \\
\hline Cs -137 & $2.31 E-02$ & $3.13 E+04$ & $3.91 E+03$ & $3.85 E+01$ & $3.64 \mathrm{E}-06$ & $2.66 E-46$ & $1.79 \mathrm{E}-96$ & $5.15 E+07$ \\
\hline $\mathrm{Ba}-137 \mathrm{~m}$ & $1.50 E+01$ & $2.95 \mathrm{E}+04$ & $0.00 E+00$ & $0.00 E+00$ & $0.00 E+00$ & $0.00 E+00$ & $0.00 E+00$ & $4.86 \mathrm{E}+07$ \\
\hline others & $1.50 \mathrm{E}+01$ & $1.69 E+03$ & $0.00 \mathrm{E}+00$ & $0.00 E+00$ & $0.00 E+00$ & $0.00 \mathrm{E}+00$ & $0.00 \mathrm{E}+00$ & $2.78 \mathrm{E}+06$ \\
\hline TOTAI & & $1.06 E+05$ & $6.36 \mathrm{E}+03$ & $5.75 \mathrm{E}+01$ & $1.40 E-01$ & $1.40 \mathrm{E}-01$ & $1.40 \mathrm{E}-01$ & $1.75 E+08$ \\
\hline & & & & & & & & \\
\hline Nuclide & Sp Activity & & g/pkg & at & year & & & \\
\hline & $(\mathrm{Ci} / \mathrm{g})$ & 10 & 100 & 300 & 1000 & & & \\
\hline $5 x-90$ & $1.36 \mathrm{E}+02$ & $1.60 E+02$ & $1.80 E+01$ & $1.39 \mathrm{E}-01$ & 5.60E-09 & & & \\
\hline$x-90$ & $5.44 \mathrm{E}+05$ & $4.01 E-02$ & $0.00 E+00$ & $0.00 \mathrm{E}+00$ & $0.00 \mathrm{E}+00$ & & & \\
\hline Cs-135 & $1.15 \mathrm{E}-03$ & $1.22 \mathrm{E}+02$ & $1.22 E+02$ & $1.22 \mathrm{E}+02$ & $1.22 E+02$ & & & \\
\hline Cs-137 & $8.70 E+01$ & $3.60 \mathrm{E}+02$ & $4.50 \mathrm{E}+01$ & $4.43 E-01$ & $4.19 E-08$ & & & \\
\hline
\end{tabular}


Table 10. Inventory of an A1-8 Package, 1 of 2

\begin{tabular}{|c|c|c|c|c|c|c|c|c|}
\hline Nuclide & Lambda & & Ci/pkg & at & year & & & stream total \\
\hline 1500 & $(1 a)$ & 10 & 100 & 300 & 1000 & 5000 & 10000 & $\mathrm{Cl}$ at 10 a \\
\hline$c-14$ & $1.21 \mathrm{E}-04$ & & & & & & & \\
\hline $\mathrm{Ni}-59$ & $9.24 \mathrm{E}-06$ & & & & & & & \\
\hline $5 e-79$ & 1.07E-05 & $1.68 E-01$ & $1.68 \mathrm{E}-01$ & 1.67E-01 & $1.66 \mathrm{E}-01$ & $1.59 \mathrm{E}-01$ & 1.51E-01 & $2.52 E+02$ \\
\hline$s x-90$ & $2.43 E-02$ & & & & & & & \\
\hline$Y-90$ & $9.48 E+01$ & & & & & & & \\
\hline $2 r-93$ & $4.53 \mathrm{E}-07$ & 7.32E-01 & $7.32 \mathrm{E}-01$ & 7.32E-01 & $7.32 E-01$ & $7.30 \mathrm{E}-01$ & $7.29 E-01$ & $1.10 \mathrm{E}+03$ \\
\hline $\mathrm{NB}-94$ & $3.41 E-05$ & & & & & & & \\
\hline TC-99 & $3.29 E-06$ & $5.27 E+00$ & $5.27 E+00$ & $5.26 E+00$ & $5.25 E+00$ & $5.18 \mathrm{E}+00$ & $5.10 E+00$ & $7.90 \mathrm{E}+03$ \\
\hline Pd-107 & $1.07 \mathrm{E}-07$ & $4.32 E-02$ & $4.32 E-02$ & $4.32 E-02$ & $4.32 \mathrm{E}-02$ & $4.32 \mathrm{E}-02$ & $4.32 \mathrm{E}-02$ & $6.48 \mathrm{E}+01$ \\
\hline $\mathrm{sn}-126$ & $6.93 E-06$ & $3.12 E-01$ & $3.12 E-01$ & $3.11 E-01$ & $3.10 \mathrm{E}-01$ & $3.01 \mathrm{E}-01$ & $2.91 \mathrm{E}-01$ & $4.68 E+02$ \\
\hline$I-129$ & $4.41 \mathrm{E}-08$ & & & & & & & \\
\hline Cs-135 & $3.01 \mathrm{E}-07$ & & & & & & & \\
\hline$C s-137$ & $2.31 E-02$ & & & & & & & \\
\hline $\mathrm{sm-151}$ & $7.70 \mathrm{E}-03$ & $1.14 E+04$ & $5.71 \mathrm{E}+03$ & $1.22 \mathrm{E}+03$ & $5.58 E+00$ & $2.33 E-13$ & $4.40 \mathrm{E}-30$ & $1.71 E+07$ \\
\hline Eu-154 & $7.88 \mathrm{E}-02$ & $1.67 E+05$ & $1.40 \mathrm{E}+02$ & 2.01E-05 & $2.28 \mathrm{E}-29$ & $3.36 E-166$ & $0.00 E+00$ & $2.51 E+08$ \\
\hline$u-234$ & $2.83 \mathrm{E}-06$ & 4.68E-02 & $4.68 \mathrm{E}-02$ & $4.68 \mathrm{E}-02$ & $4.67 \mathrm{E}-02$ & $4.62 E-02$ & $4.55 \mathrm{E}-02$ & $7.02 E+01$ \\
\hline $\mathrm{U}-235$ & $9.76 \mathrm{E}-10$ & $8.02 E-04$ & 8.02E-04 & $8.02 E-04$ & $8.02 E-04$ & $8.02 E-04$ & $8.02 E-04$ & $1.20 E+00$ \\
\hline $\mathrm{U}-236$ & $2.96 \mathrm{E}-08$ & $1.04 \mathrm{E}-02$ & $1.04 E-02$ & $1.04 \mathrm{E}-02$ & $1.04 \mathrm{E}-02$ & $1.04 \mathrm{E}-02$ & $1.04 \mathrm{E}-02$ & $1.55 E+01$ \\
\hline$u-238$ & $1.55 \mathrm{E}-09$ & $1.27 \mathrm{E}-02$ & 1.27E-02 & $1.27 \mathrm{E}-02$ & 1.27E-02 & $1.27 \mathrm{E}-02$ & 1.27E-02 & $1.91 E+01$ \\
\hline$T h-230$ & $9.00 \mathrm{E}-05$ & & $5.35 \mathrm{E}-04$ & $5.52 \mathrm{E}-03$ & $6.29 \mathrm{E}-02$ & $1.42 \mathrm{E}+00$ & $4.95 E+00$ & $0.00 E+00$ \\
\hline$R a-226$ & $4.33 \mathrm{E}-04$ & & $1.59 \mathrm{E}-02$ & $5.18 \mathrm{E}-01$ & $1.89 E+01$ & $1.53 E+03$ & $7.64 E+03$ & $0.00 E+00$ \\
\hline Np-237 & $3.24 \mathrm{E}-07$ & 1.27E-02 & $4.83 E+02$ & $1.61 E+03$ & $3.63 E+03$ & $4.60 \mathrm{E}+03$ & $4.59 E+03$ & $1.91 E+01$ \\
\hline $\mathrm{Pu}-238$ & $7.90 \mathrm{E}-03$ & $9.03 E+01$ & $4.43 E+01$ & $9.13 \mathrm{E}+00$ & $3.62 \mathrm{E}-02$ & $6.84 E-16$ & $4.79 \mathrm{E}-33$ & $1.35 \mathrm{E}+05$ \\
\hline $\mathrm{Pu}-239$ & $2.88 E-05$ & $1.27 E+01$ & $1.27 E+01$ & $1.26 \mathrm{E}+01$ & $1.23 E+01$ & $1.10 E+01$ & $9.53 E+00$ & $1.91 E+04$ \\
\hline Pu-240 & $1.06 \mathrm{E}-04$ & $2.14 E+01$ & $2.12 E+01$ & $2.07 E+01$ & $1.93 \mathrm{E}+01$ & $1.26 \mathrm{E}+01$ & $7.45 \mathrm{E}+00$ & $3.21 E+04$ \\
\hline $\mathrm{Pu}-241$ & $4.81 \mathrm{E}-02$ & $3.08 E+03$ & $4.04 E+01$ & $2.66 \mathrm{E}-03$ & $6.20 \mathrm{E}-18$ & $1.49 \mathrm{E}-101$ & $4.45 \mathrm{E}-206$ & $4.61 E+06$ \\
\hline Pu-242 & $1.86 \mathrm{E}-06$ & $7.02 E-02$ & $7.02 E-02$ & $7.02 E-02$ & $7.01 \mathrm{E}-02$ & $6.96 \mathrm{E}-02$ & 6.89E-02 & $1.05 E+02$ \\
\hline Am-241 & $1.60 \mathrm{E}-03$ & $7.35 E+01$ & $2.86 E+03$ & $2.05 E+03$ & $6.66 \mathrm{E}+02$ & $1.10 \mathrm{E}+00$ & $3.65 E-04$ & $1.10 E+05$ \\
\hline$A m-242 m$ & 4.92E-03 & $2.41 \mathrm{E}-01$ & $1.55 \mathrm{E}-01$ & $5.79 \mathrm{E}-02$ & $1.85 \mathrm{E}-03$ & $5.35 E-12$ & $1.13 \mathrm{E}-22$ & $3.61 \mathrm{E}+02$ \\
\hline$A m-243$ & 9.39E-05 & 7.02E-01 & $6.96 \mathrm{E}-01$ & $6.83 E-01$ & $6.40 \mathrm{E}-01$ & 4.39E-01 & $2.75 E-01$ & $1.05 E+03$ \\
\hline $\mathrm{Cm}-242$ & $1.55 \mathrm{E}+00$ & $1.97 \mathrm{E}-01$ & $4.01 \mathrm{E}-62$ & $5.41 \mathrm{E}-197$ & $0.00 E+00$ & $0.00 E+00$ & $0.00 E+00$ & $2.96 \mathrm{E}+02$ \\
\hline$C m-244$ & $3.83 E-02$ & $5.35 \mathrm{E}+01$ & $1.70 E+00$ & $8.04 \mathrm{E}-04$ & $1.83 \mathrm{E}-15$ & $5.46 \mathrm{E}-82$ & $3.80 E-165$ & $8.02 E+04$ \\
\hline $\mathrm{Cm}-245$ & $8.15 \mathrm{E}-05$ & $6.02 E-03$ & $5.97 \mathrm{E}-03$ & $5.88 \mathrm{E}-03$ & $5.55 \mathrm{E}-03$ & $4.01 E-03$ & $2.66 \mathrm{E}-03$ & $9.03 E+00$ \\
\hline$C m-246$ & $1.47 \mathrm{E}-04$ & $1.24 \mathrm{E}-03$ & $1.22 \mathrm{E}-03$ & $1.19 \mathrm{E}-03$ & $1.07 \mathrm{E}-03$ & $5.95 \mathrm{E}-04$ & $2.86 \mathrm{E}-04$ & $1.86 \mathrm{E}+00$ \\
\hline others & & $3.83 E+05$ & & & & & & $5.75 E+08$ \\
\hline total & & $5.65 E+05$ & $9.32 E+03$ & $4.93 E+03$ & $4.36 E+03$ & $6.16 \mathrm{E}+03$ & $1.23 E+04$ & $8.48 E+08$ \\
\hline
\end{tabular}


Table 10. Inventory of an A1-8 Package, 2 of 2

\begin{tabular}{|c|c|c|c|c|c|c|c|c|}
\hline Nuclide & Sp Activity & & g/pkg & at & year & & & stream total \\
\hline 1500 & $(\mathrm{C} 1 / \mathrm{g})$ & 10 & 100 & 300 & 1000 & 5000 & 10000 & $\mathrm{Cl}$ at 1000 a \\
\hline$c-14$ & $4.46 \mathrm{E}+00$ & & & & & & & \\
\hline $\mathrm{Ni}-59$ & $7.57 E+04$ & & & & & & & \\
\hline Se-79 & $6.97 \mathrm{E}-02$ & $2.41 E+00$ & $2.41 E+00$ & $2.40 E+00$ & $2.39 E+00$ & $2.29 E+00$ & $2.17 E+00$ & $2.49 E+0$ \\
\hline $5 x-90$ & $1.36 \mathrm{E}+02$ & & & & & & & \\
\hline $\mathbf{Y}-90$ & $5.44 E+05$ & & & & & & & \\
\hline $2 x-93$ & 2.51E-03 & $2.92 E+02$ & $2.92 \mathrm{E}+02$ & $2.92 E+02$ & $2.92 E+02$ & $2.91 E+02$ & $2.90 \mathrm{E}+02$ & $1.10 \mathrm{E}+0$ \\
\hline $\mathrm{Mb}-94$ & 1.87E-01 & & & & & & & \\
\hline Tc-99 & 1.70E-02 & $3.11 E+02$ & $3.11 \mathrm{E}+02$ & $3.11 E+02$ & $3.10 \mathrm{E}+02$ & $3.06 \mathrm{E}+02$ & $3.01 E+02$ & $7.88 E+03$ \\
\hline Pd-107 & $5.14 E-04$ & $8.40 E+01$ & $8.40 E+01$ & $8.40 E+01$ & $8.40 E+01$ & $8.40 E+01$ & $8.40 E+01$ & $6.48 E+01$ \\
\hline $5 n-126$ & $2.84 \mathrm{E}-02$ & $1.10 E+01$ & $1.10 E+01$ & $1.10 E+01$ & $1.09 E+01$ & $1.06 \mathrm{E}+01$ & $1.03 E+01$ & $4.65 E+02$ \\
\hline$I-129$ & 1.77E-04 & & & & & & & \\
\hline Cs-135 & $1.15 E-03$ & & & & & & & \\
\hline Cs-137 & $8.70 E+01$ & & & & & & & \\
\hline $5 m-151$ & $2.63 E+01$ & $4.34 E+02$ & $2.17 E+02$ & $4.65 E+01$ & 2.12E-01 & $8.86 \mathrm{E}-15$ & $1.67 \mathrm{E}-31$ & $8.36 \mathrm{E}+03$ \\
\hline$E u-154$ & $2.70 E+02$ & $6.20 \mathrm{E}+02$ & 5.17E-01 & $7.45 \mathrm{E}-08$ & $8.44 \mathrm{E}-32$ & $1.24 \mathrm{E}-168$ & $0.00 \mathrm{E}+00$ & $3.42 \mathrm{E}-26$ \\
\hline $0-234$ & $6.25 \mathrm{E}-03$ & $7.49 E+00$ & $7.49 \mathrm{E}+00$ & $7.48 E+00$ & $7.47 E+00$ & $7.39 E+00$ & $7.28 E+00$ & $7.00 E+01$ \\
\hline u-235 & 2.16E-06 & $3.71 E+02$ & $3.71 E+02$ & $3.71 E+02$ & $3.71 E+02$ & $3.71 E+02$ & $3.71 E+02$ & $1.20 E+00$ \\
\hline $0-236$ & $6.47 \mathrm{E}-05$ & $1.60 E+02$ & $1.60 E+02$ & $1.60 E+02$ & $1.60 \mathrm{E}+02$ & $1.60 \mathrm{E}+02$ & $1.60 E+02$ & $1.55 E+01$ \\
\hline$U-238$ & $3.36 \mathrm{E}-07$ & $3.78 E+04$ & $3.78 E+04$ & $3.78 E+04$ & $3.78 E+04$ & $3.78 E+04$ & $3.78 E+04$ & $1.91 E+01$ \\
\hline Th-230 & $2.02 E-01$ & & $2.65 \mathrm{E}-03$ & 2.73E-02 & 3.12E-01 & $7.03 E+00$ & $2.45 E+01$ & $9.44 E+01$ \\
\hline Ra-226 & $9.88 \mathrm{E}-01$ & & $1.61 \mathrm{E}-02$ & 5.25E-01 & $1.91 E+01$ & $1.55 E+03$ & $7.74 \mathrm{E}+03$ & $2.83 E+04$ \\
\hline Np-237 & 7.05E-04 & $1.80 E+01$ & $6.85 E+05$ & $2.28 \mathrm{E}+06$ & $5.15 E+06$ & $6.52 E+06$ & $6.51 E+06$ & $5.44 E+06$ \\
\hline Pu-238 & $1.71 E+01$ & $5.27 E+00$ & $2.59 E+00$ & 5.33E-01 & $2.12 \mathrm{E}-03$ & $4.00 \mathrm{E}-17$ & $2.80 E-34$ & $5.43 E+01$ \\
\hline Pu-239 & $6.22 \mathrm{E}-02$ & $2.04 E+02$ & $2.04 E+02$ & $2.03 E+02$ & $1.98 E+02$ & $1.77 \mathrm{E}+02$ & $1.53 \mathrm{E}+02$ & $1.85 E+04$ \\
\hline Pu-240 & $2.28 \mathrm{E}-01$ & $9.38 E+01$ & $9.29 \mathrm{E}+01$ & $9.10 \mathrm{E}+01$ & $8.45 \mathrm{E}+01$ & $5.54 E+01$ & $3.27 E+01$ & $2.89 E+04$ \\
\hline Pu-241 & $1.03 E+02$ & $2.99 E+01$ & 3.92E-01 & 2.59E-05 & $6.02 E-20$ & $1.44 \mathrm{E}-103$ & 4.32E-208 & $9.29 \mathrm{E}-15$ \\
\hline $\mathrm{Pu}-242$ & $3.82 E-03$ & $1.84 E+01$ & $1.84 E+01$ & $1.84 E+01$ & $1.83 E+01$ & $1.82 E+01$ & $1.80 E+01$ & $1.05 E+02$ \\
\hline Am-241 & $3.43 E+00$ & $2.14 E+01$ & $8.34 \mathrm{E}+02$ & $5.96 \mathrm{E}+02$ & $1.94 E+02$ & $3.20 \mathrm{E}-01$ & $1.06 \mathrm{E}-04$ & $1.00 E+06$ \\
\hline$A m-242 m$ & $9.72 E+00$ & $2.48 \mathrm{E}-02$ & $1.59 \mathrm{E}-02$ & $5.95 \mathrm{E}-03$ & $1.91 \mathrm{E}-04$ & $5.50 \mathrm{E}-13$ & $1.16 \mathrm{E}-23$ & $2.78 E+00$ \\
\hline$A m-243$ & 1.99E-01 & $3.53 E+00$ & $3.50 \mathrm{E}+00$ & $3.43 E+00$ & $3.21 E+00$ & $2.21 E+00$ & $1.38 \mathrm{E}+00$ & $9.60 E+02$ \\
\hline $\mathrm{Cm}-242$ & $3.31 E+03$ & $5.96 \mathrm{E}-05$ & $1.21 \mathrm{E}-65$ & $1.64 \mathrm{E}-200$ & $0.00 \mathrm{E}+00$ & $0.00 E+00$ & $0.00 E+00$ & $0.00 E+00$ \\
\hline$c m-244$ & $8.09 E+01$ & 6.61E.01 & $2.11 \mathrm{E}-02$ & $9.93 \mathrm{E}-06$ & $2.27 \mathrm{E}-17$ & $6.75 \mathrm{E}-84$ & $4.70 \mathrm{E}-167$ & $2.75 \mathrm{E}-12$ \\
\hline$C m-245$ & $1.72 E-01$ & $3.50 \mathrm{E}-02$ & $3.47 \mathrm{E}-02$ & $3.42 \mathrm{E}-02$ & $3.23 E-02$ & $2.33 \mathrm{E}-02$ & $1.55 \mathrm{E}-02$ & $8.33 E+00$ \\
\hline $\mathrm{Cm}-246$ & $3.07 \mathrm{E}-01$ & $4.03 E-03$ & $3.98 \mathrm{E}-03$ & $3.86 \mathrm{E}-03$ & $3.48 E-03$ & $1.94 E-03$ & $9.32 E-04$ & $1.60 E+00$ \\
\hline
\end{tabular}


Table 11. Inventory of an A3-2,5 Package, 1 of 2

\begin{tabular}{|c|c|c|c|c|c|c|c|c|c|}
\hline Nuclide & Lambda & & Ci/pkg & at & year & & & stream & total \\
\hline 5112 & $(1 a)$ & 10 & 100 & 300 & 1000 & 5000 & 10000 & $\mathrm{Cl}$ at 10 a & $\mathrm{Cl}$ at $1000 \mathrm{a}$ \\
\hline $\mathrm{Ni}-59$ & $9.24 \mathrm{E}-06$ & $3.59 E+00$ & $3.59 \mathrm{E}+00$ & $3.58 \mathrm{E}+00$ & $3.56 \mathrm{E}+00$ & $3.43 E+00$ & $3.28 E+00$ & $1.84 E+04$ & $1.82 E+04$ \\
\hline Se-79 & $1.07 \mathrm{E}-05$ & $4.11 E+00$ & $4.11 \mathrm{E}+00$ & $4.10 E+00$ & $4.07 E+00$ & $3.90 \mathrm{E}+00$ & $3.69 E+00$ & $2.10 \mathrm{E}+04$ & $2.08 E+04$ \\
\hline $5 x-90$ & $2.43 E-02$ & $0.00 \mathrm{E}+00$ & $0.00 E+00$ & $0.00 E+00$ & $0.00 E+00$ & $0.00 E+00$ & $0.00 E+00$ & $0.00 \mathrm{E}+00$ & $0.00 E+00$ \\
\hline$Y-90$ & $9.48 E+01$ & $0.00 E+00$ & $0.00 E+00$ & $0.00 E+00$ & $0.00 E+00$ & $0.00 E+00$ & $0.00 \mathrm{E}+00$ & $0.00 \mathrm{E}+00$ & $0.00 \mathrm{E}+00$ \\
\hline$z x-93$ & $4.53 E-07$ & $0.00 E+00$ & $0.00 E+00$ & $0.00 E+00$ & $0.00 E+00$ & $0.00 E+00$ & $0.00 E+00$ & $0.00 E+00$ & $0.00 E+00$ \\
\hline $\mathrm{Nb}-94$ & $3.41 E-05$ & $9.17 \mathrm{E}-03$ & $9.14 \mathrm{E}-03$ & $9.08 \mathrm{E}-03$ & $8.86 \mathrm{E}-03$ & $7.73 \mathrm{E}-03$ & $6.52 \mathrm{E}-03$ & $59 E+01$ & $3 \mathrm{E}+01$ \\
\hline Tc-99 & $3.29 E-06$ & $1.41 E+02$ & $1.41 \mathrm{E}+02$ & $1.41 E+02$ & $1.41 E+02$ & $1.39 E+02$ & $1.37 E+02$ & $7.22 E+05$ & $7.20 E+05$ \\
\hline Pd-107 & $1.07 \mathrm{E}-07$ & $2.29 E+00$ & $2.29 E+00$ & $2.29 E+00$ & $2.29 E+00$ & $2.29 E+00$ & $2.29 E+00$ & $1.17 E+04$ & $E+04$ \\
\hline $5 n-126$ & $6.93 \mathrm{E}-06$ & $1.73 E+01$ & $1.73 E+01$ & $1.73 E+01$ & $1.72 E+01$ & $1.67 E+01$ & $1.62 E+01$ & $8.85 E+04$ & $8.79 E+04$ \\
\hline $\mathrm{U}-234$ & $2.83 \mathrm{E} \cdot 06$ & $3.37 \mathrm{E}-02$ & $3.37 E-02$ & $3.37 \mathrm{E}-02$ & $3.36 \mathrm{E}-02$ & $3.33 \mathrm{E}-02$ & $E-02$ & $E+02$ & $E+02$ \\
\hline $\mathrm{U}-235$ & -10 & $1.69 \mathrm{E}-05$ & E-05 & $9 \mathrm{E}-05$ & $1.69 \mathrm{E}-05$ & $E-05$ & .05 & $=-02$ & 2E-02 \\
\hline & $2.96 \mathrm{E}-08$ & $2.02 E-04$ & $2.02 E-04$ & $2.02 E-04$ & 2.02E-04 & $2 E-04$ & $E-04$ & $3 E+00$ & $3 E+00$ \\
\hline$U-238$ & .09 & $2.10 \mathrm{E}-03$ & -03 & $2.10 \mathrm{E}-03$ & $2.10 \mathrm{E}-03$ & E-03 & -03 & $E+01$ & $E+01$ \\
\hline Th-230 & -05 & $0.00 \mathrm{E}+00$ & .04 & $3.98 \mathrm{E}-03$ & $4.54 \mathrm{E}-02$ & $1.02 E+00$ & $3.57 \mathrm{E}+00$ & $0.00 E+00$ & $2.32 E+02$ \\
\hline$R a-226$ & $4.33 E-04$ & $0.00 E+00$ & $1.15 \mathrm{E}-02$ & $3.73 E-01$ & $1.36 E+01$ & $E+03$ & +03 & $E+00$ & $5 E+04$ \\
\hline$N p-237$ & $3.24 E-07$ & $7.71 \mathrm{E}-03$ & $E+02$ & $1.34 E+03$ & $3.03 E+03$ & $3 E+03$ & $E+03$ & $3.94 E+01$ & $1.55 E+07$ \\
\hline$P u-238$ & $7.90 E-03$ & $3.37 E+02$ & $1.66 \mathrm{E}+02$ & $3.41 \mathrm{E}+01$ & $1.35 \mathrm{E}-01$ & $E-15$ & -32 & $1.72 \mathrm{E}+06$ & $6.92 E+02$ \\
\hline Pu-239 & $2.88 E-05$ & $4.58 E+01$ & $4.56 \mathrm{E}+01$ & $4.54 E+01$ & $4.45 E+01$ & $E+01$ & +01 & $4 E+05$ & $E+05$ \\
\hline$P u-240$ & $1.06 \mathrm{E}-04$ & $6.02 E+01$ & $5.96 E+01$ & $5.84 E+01$ & $5.42 E+01$ & $3.55 \mathrm{E}+01$ & $2.10 \mathrm{E}+01$ & $3.08 \mathrm{E}+05$ & $2.77 E+05$ \\
\hline Pu-241 & $4.81 \mathrm{E}-02$ & $1.64 E+03$ & $2.15 \mathrm{E}+01$ & $1.42 \mathrm{E}-03$ & $3.30 E-18$ & $E-102$ & $E-206$ & $7 E+06$ & $9 E-14$ \\
\hline Pu-242 & $1.86 \mathrm{E}-06$ & $1.16 \mathrm{E}-01$ & $1.16 \mathrm{E}-01$ & $1.16 \mathrm{E}-01$ & 1.15E-01 & E-01 & $=-01$ & $5.91 E+02$ & $5.90 E+02$ \\
\hline Am-241 & $1.60 E-03$ & $1.06 E+02$ & $1.58 E+03$ & $1.13 E+03$ & $3.69 E+02$ & $6.08 \mathrm{E}-01$ & 2.02E-04 & $5.42 \mathrm{E}+05$ & $1.88 E+06$ \\
\hline$A m-242 m$ & $4.92 E-03$ & $1.59 E+01$ & $1.02 E+01$ & $3.82 E+00$ & $1.22 E-01$ & $3.53 E-10$ & $7.47 \mathrm{E}-21$ & $8.13 E+04$ & $E+02$ \\
\hline $\mathrm{Am}-243$ & $9.39 \mathrm{E}-05$ & $2.10 E+00$ & $2.08 E+00$ & $2.04 E+00$ & $1.91 E+00$ & $1.31 \mathrm{E}+00$ & $8.20 \mathrm{E}-01$ & $1.07 E+04$ & $9.76 E+03$ \\
\hline$C n-242$ & $1.55 E+00$ & $1.32 E+01$ & $2.70 \mathrm{E}-60$ & $3.64 \mathrm{E}-195$ & $0.00 E+00$ & $0.00 \mathrm{E}+00$ & $0.00 \mathrm{E}+00$ & $6.77 \mathrm{E}+04$ & $0.00 E+00$ \\
\hline Cm-244 & $3.83 E-02$ & $1.71 \mathrm{E}+02$ & $5.45 E+00$ & 2.57E-03 & $5.86 E-15$ & $1.75 \mathrm{E}-81$ & $1.21 \mathrm{E}-164$ & $8.74 E+05$ & $3.00 E-11$ \\
\hline $\mathrm{Cm}-245$ & $8.15 E-05$ & $1.11 \mathrm{E}-01$ & $1.10 \mathrm{E}-01$ & $1.08 \mathrm{E}-01$ & $1.02 \mathrm{E}-01$ & $7.38 \mathrm{E}-02$ & $4.91 E-02$ & $5.66 \mathrm{E}+02$ & $5.22 E+02$ \\
\hline $\mathrm{Cm}-246$ & $1.47 \mathrm{E}-04$ & $3.85 E-02$ & $3.80 \mathrm{E}-02$ & $3.69 E-02$ & $3.33 E-02$ & $1.85 \mathrm{E}-02$ & 8.91E-03 & $1.97 E+02$ & $1.70 E+02$ \\
\hline $\mathrm{Ba}-137 \mathrm{~m}$ & $1.50 E+01$ & $3.85 E-02$ & $0.00 E+00$ & $0.00 E+00$ & $0.00 \mathrm{E}+00$ & $0.00 \mathrm{E}+00$ & $0.00 \mathrm{E}+00$ & $1.97 \mathrm{E}+02$ & \\
\hline total & & $2.56 \mathrm{E}+03$ & $2.46 \mathrm{E}+03$ & $2.79 E+03$ & $3.68 E+03$ & $5.18 \mathrm{E}+03$ & $9.56 \mathrm{E}+03$ & $1.31 E+07$ & \\
\hline
\end{tabular}




\begin{tabular}{|c|c|c|c|c|c|c|c|}
\hline Nuclide & Sp Activity & & g/pkg & at & year & & \\
\hline 5112 & $(\mathrm{Ci} / \mathrm{g})$ & 10 & 100 & 300 & 1000 & 5000 & 10000 \\
\hline $\mathrm{Ni}-59$ & $7.57 E+04$ & $4.74 E-05$ & $.74 E-05$ & $4.73 E-05$ & $4.70 E-05$ & $4.53 E-05$ & $3 E-05$ \\
\hline se-79 & $.97 E-02$ & $5.90 E+01$ & $5.89 E+01$ & $88 \mathrm{E}+01$ & $5.84 \mathrm{E}+01$ & $5.59 E+01$ & $5.30 E+01$ \\
\hline$s x-9$ & $1.36 \mathrm{E}+02$ & $0.00 \mathrm{E}+00$ & $0.00 E+00$ & $0.00 E+00$ & $0.00 \mathrm{E}+00$ & $0.00 \mathrm{E}+00$ & $.00 E+00$ \\
\hline$y-90$ & $5.44 E+05$ & $0.00 E+00$ & $0.00 \mathrm{E}+00$ & $0.00 E+00$ & $0.00 \mathrm{E}+00$ & & $0 E+00$ \\
\hline $2 x-93$ & & & $0.00 \mathrm{E}+00$ & $0.00 \mathrm{E}+00$ & $0.00 E+00$ & $0.00 \mathrm{E}+00$ & $E+00$ \\
\hline $\mathrm{Nb}-94$ & $7 E-01$ & $4.89 E-02$ & $4.88 \mathrm{E}-02$ & $4.85 \mathrm{E}-02$ & $4.73 E-02$ & $4.13 E-02$ & \\
\hline Tc-99 & & & & +03 & $=+03$ & +03 & +03 \\
\hline$P d-107$ & & 03 & & $E+03$ & $4.46 \mathrm{E}+03$ & & $=03$ \\
\hline Sn- & 02 & +02 & & & +02 & & +02 \\
\hline $\mathrm{U}-234$ & & +00 & & 00 & +00 & & +00 \\
\hline $\mathrm{U}-2$ & & 00 & 00 & +00 & $E+00$ & +00 & $8+00$ \\
\hline $\mathrm{U}-\mathrm{z}$ & & & & & $E+00$ & & \\
\hline $\mathrm{U}-238$ & & +03 & & & $E+03$ & & $E+03$ \\
\hline Th-230 & & & & -02 & E-01 & $=00$ & $E+01$ \\
\hline$R a-226$ & & & & -01 & $E+01$ & +03 & +03 \\
\hline Np-237 & & +01 & & & $E+06$ & +06 & $E+06$ \\
\hline $\mathrm{Pu}-238$ & +01 & +01 & +00 & & E-03 & & E-33 \\
\hline Pu-239 & & +02 & +02 & +02 & $E+02$ & +02 & $E+02$ \\
\hline$P u-240$ & -01 & $2.64 E+02$ & & & $\mathrm{E}+02$ & 02 & $E+01$ \\
\hline$P u-241$ & $3 E+02$ & $1.59 \mathrm{E}+01$ & & & $E-20$ & & -208 \\
\hline $\mathrm{Pu}-242$ & 03 & +01 & .01 & +01 & $2 E+01$ & $E+01$ & $\bar{E}+01$ \\
\hline Am-241 & $3 E+00$ & $3.09 \mathrm{E}+01$ & +02 & $3.30 E+02$ & $1.07 E+02$ & & 5.89E-05 \\
\hline Am-242m & & & & & $=-02$ & & $E-22$ \\
\hline $\mathrm{Am}-243$ & $1.99 E-01$ & $1.05 E+01$ & $1.04 E+01$ & $2 E+01$ & $9.60 \mathrm{E}+00$ & $E+00$ & $E+00$ \\
\hline$C m-242$ & $3.31 E+03$ & & & & $0.00 \mathrm{E}+00$ & & $\mathrm{E}+00$ \\
\hline$C m-244$ & $8.09 E+01$ & $2.11 E+00$ & & -05 & $7.24 \mathrm{E}-17$ & $E-83$ & -166 \\
\hline$c m-245$ & $1.72 \mathrm{E}-01$ & & & & $E-01$ & & E-01 \\
\hline$m-246$ & $3.07 E-01$ & $1.26 \mathrm{E}-01$ & -01 & $1.20 \mathrm{E}-01$ & $1.09 E-01$ & $6.04 \mathrm{E}-02$ & 2.9 \\
\hline
\end{tabular}


Table 12. Inventory of an A3-4 Package

\begin{tabular}{|c|c|c|c|c|c|c|c|}
\hline Nuclide & Lambda & & & $\mathrm{Ci} / \mathrm{pkg}$ at $\mathrm{yr}$ & & & \\
\hline 16880 & (1a) & 10 & 100 & 300 & 1000 & 5000 & 10000 \\
\hline $\mathrm{Sr}-90$ & 2.43E-02 & $9.32 \mathrm{E}+04$ & $1.04 E+04$ & $8.06 \mathrm{E}+01$ & $3.26 \mathrm{E}-06$ & $1.83 E-48$ & $2.82 \mathrm{E}-101$ \\
\hline$Y-90$ & $9.48 E+01$ & $9.32 E+04$ & $0.00 \mathrm{E}+00$ & $0.00 \mathrm{E}+00$ & $0.00 \mathrm{E}+00$ & $0.00 E+00$ & $0.00 E+00$ \\
\hline$I-129$ & $4.41 E-08$ & $1.50 \mathrm{E}-01$ & $1.50 \mathrm{E}-01$ & $1.50 \mathrm{E}-01$ & $1.50 \mathrm{E}-01$ & $1.50 \mathrm{E}-01$ & $1.50 \mathrm{E}-01$ \\
\hline Cs-135 & $3.01 \mathrm{E}-07$ & $5.18 \mathrm{E}+00$ & $5.18 \mathrm{E}+00$ & $5.18 \mathrm{E}+00$ & $5.18 \mathrm{E}+00$ & $5.17 \mathrm{E}+00$ & $5.16 \mathrm{E}+00$ \\
\hline $\mathrm{Cs}-137$ & $2.31 \mathrm{E}-02$ & $2.47 E+05$ & $3.08 E+04$ & $3.03 E+02$ & $2.87 E-05$ & $2.09 \mathrm{E}-45$ & $1.41 \mathrm{E}-95$ \\
\hline $\mathrm{Ba}-137 \mathrm{~m}$ & $1.50 \mathrm{E}+01$ & $2.33 E+05$ & $0.00 \mathrm{E}+00$ & $0.00 \mathrm{E}+00$ & $0.00 E+00$ & $0.00 E+00$ & $0.00 E+00$ \\
\hline others & & $8.61 E+03$ & & & & & \\
\hline TOTAL & & $4.88 E+05$ & $3.08 E+04$ & $3.09 \mathrm{E}+02$ & $5.33 E+00$ & $5.32 E+00$ & $5.31 E+00$ \\
\hline & & & & & & & \\
\hline \multirow[t]{2}{*}{ Nuclide } & Sp Activity & & & g/pkg at yr & & stream & total \\
\hline & $(\mathrm{Ci} / \mathrm{g})$ & 10 & 100 & 300 & 1000 & $\mathrm{Ci}$ at $10 \mathrm{a}$ & at $1000 \mathrm{a}$ \\
\hline $5 x-90$ & $1.36 \mathrm{E}+02$ & $6.86 \mathrm{E}+02$ & $7.68 \mathrm{E}+01$ & $5.93 E-01$ & $2.39 E-08$ & $1.57 \mathrm{E}+09$ & $5.50 \mathrm{E}-02$ \\
\hline $\mathbf{Y - 9 0}$ & $5.44 \mathrm{E}+05$ & $1.71 \mathrm{E}-01$ & $0.00 \mathrm{E}+00$ & $0.00 E+00$ & $0.00 \mathrm{E}+00$ & $1.57 E+09$ & $0.00 \mathrm{E}+00$ \\
\hline$I-129$ & $1.77 \mathrm{E}-04$ & $8.47 E+02$ & $8.47 E+02$ & $8.47 E+02$ & $8.47 E+02$ & $2.53 E+03$ & $2.53 E+03$ \\
\hline Cs-135 & $1.15 \mathrm{E}-03$ & $4.50 E+03$ & $4.50 E+03$ & $4.50 \mathrm{E}+03$ & $4.50 \mathrm{E}+03$ & $8.74 E+04$ & $8.74 E+04$ \\
\hline Cs-137 & $8.70 \mathrm{E}+01$ & $2.83 E+03$ & $3.54 E+02$ & $3.49 \mathrm{E}+00$ & $3.30 \mathrm{E}-07$ & $4.16 \mathrm{E}+09$ & $4.84 \mathrm{E}-01$ \\
\hline $\mathrm{Ba}-137 \mathrm{~m}$ & & & & & & $3.93 E+09$ & \\
\hline others & & & & & & $1.45 E+08$ & \\
\hline TOTAL & & $8.19 E+03$ & $5.71 E+03$ & $5.35 \mathrm{E}+03$ & $5.35 \mathrm{E}+03$ & $1.14 E+10$ & $8.99 E+04$ \\
\hline
\end{tabular}


Table 13. Inventory of a B1-2 Package, 1 of 2

\begin{tabular}{|c|c|c|c|c|c|c|c|c|c|}
\hline Nuclide & Lambda & & Ci/pkg & $a t$ & year & & & stream & total \\
\hline 4191 & $(/ \mathbf{a})$ & 10 & 100 & 300 & 1000 & 5000 & 10000 & $\mathrm{Ci}$ at 10 a & $\mathrm{Ci}$ at 1000 a \\
\hline$c-14$ & $1.21 \mathrm{E}-04$ & $2.39 E+01$ & $2.36 \mathrm{E}+01$ & $2.30 E+01$ & $2.12 \mathrm{E}+01$ & $1.31 \mathrm{E}+01$ & $7.13 E+00$ & $1.00 E+05$ & $8.87 E+04$ \\
\hline $\mathrm{Ni}-59$ & $9.24 \mathrm{E}-06$ & $9.09 E+01$ & $9.08 \mathrm{E}+01$ & $9.06 E+01$ & $9.00 E+01$ & $8.68 E+01$ & $8.29 E+01$ & $3.81 E+05$ & $3.77 \mathrm{E}+05$ \\
\hline$z x-93$ & $4.53 \mathrm{E}-07$ & $1.99 \mathrm{E}+00$ & $1.99 E+00$ & $1.99 \mathrm{E}+00$ & $1.99 E+00$ & $1.99 E+00$ & $1.98 \mathrm{E}+00$ & $8.34 E+03$ & $8.34 E+03$ \\
\hline $\mathrm{Mb}-94$ & $3.41 \mathrm{E}-05$ & $1.91 E+01$ & $1.90 \mathrm{E}+01$ & $1.89 E+01$ & $1.85 E+01$ & $1.61 \mathrm{E}+01$ & $1.36 \mathrm{E}+01$ & $8.00 E+04$ & $7.74 E+04$ \\
\hline TC-99 & $3.29 \mathrm{E}-06$ & $1.65 \mathrm{E}-02$ & $1.65 \mathrm{E}-02$ & $1.65 \mathrm{E}-02$ & 1.64E-02 & 1.62E-02 & $1.60 \mathrm{E}-02$ & $6.92 \mathrm{E}+01$ & $6.89 E+01$ \\
\hline $\mathrm{U}-234$ & $2.83 E-06$ & $1.79 \mathrm{E}-02$ & $1.79 \mathrm{E}-02$ & $1.79 \mathrm{E}-02$ & 1.79E-02 & 1.77E-02 & $1.74 \mathrm{E}-02$ & $7.52 \mathrm{E}+01$ & $7.50 E+01$ \\
\hline $\mathrm{U}-235$ & $9.76 \mathrm{E}-10$ & 2.57E-04 & 2.57E-04 & $2.57 \mathrm{E}-04$ & 2.57E-04 & 2.57E-04 & 2.57E-04 & $1.08 E+00$ & $1.08 \mathrm{E}+00$ \\
\hline $\mathrm{u}-236$ & $2.96 \mathrm{E}-08$ & $3.86 \mathrm{E}-03$ & $3.86 \mathrm{E}-03$ & $3.86 \mathrm{E}-03$ & $3.86 \mathrm{E}-03$ & $3.85 \mathrm{E}-03$ & $3.85 \mathrm{E}-03$ & $1.62 E+01$ & $1.62 E+01$ \\
\hline $0-238$ & $1.55 E-09$ & 4.77E-03 & 4.77E-03 & 4.77E-03 & 4.77E-03 & 4.77E-03 & 4.77E-03 & $2.00 E+01$ & $2.00 E+01$ \\
\hline $\mathrm{Th}-230$ & $9.00 \mathrm{E}-05$ & $0.00 E+00$ & $2.05 \mathrm{E}-04$ & $2.12 \mathrm{E}-03$ & $2.41 \mathrm{E}-02$ & 5.44E-01 & $1.90 \mathrm{E}+00$ & $0.00 E+00$ & $1.01 E+02$ \\
\hline$R a-226$ & 4.33E-04 & $0.00 E+00$ & $6.09 \mathrm{E}-03$ & $1.99 \mathrm{E}-01$ & $7.23 E+00$ & $5.86 \mathrm{E}+02$ & $2.93 E+03$ & $0.00 \mathrm{E}+00$ & $3.03 E+04$ \\
\hline$N p-237$ & $3.24 \mathrm{E}-07$ & $4.75 \mathrm{E}-03$ & $1.78 \mathrm{E}+02$ & $5.92 \mathrm{E}+02$ & $1.33 E+03$ & $1.69 \mathrm{E}+03$ & $1.69 E+03$ & $1.99 E+01$ & $5.59 E+06$ \\
\hline $\mathrm{Pu}-238$ & $7.90 \mathrm{E}-03$ & $3.35 \mathrm{E}+01$ & $1.64 \mathrm{E}+01$ & $3.39 \mathrm{E}+00$ & 1.34E-02 & 2.54E-16 & $1.78 \mathrm{E}-33$ & $1.40 \mathrm{E}+05$ & $5.63 E+01$ \\
\hline $\mathrm{Pu}-239$ & $2.88 E-05$ & $4.70 E+00$ & $4.69 \mathrm{E}+00$ & $4.66 \mathrm{E}+00$ & $4.57 E+00$ & $4.07 E+00$ & $3.53 E+00$ & $1.97 E+04$ & $1.92 E+04$ \\
\hline Pu-240 & $1.06 \mathrm{E}-04$ & $7.88 E+00$ & $7.81 \mathrm{E}+00$ & $7.64 \mathrm{E}+00$ & $7.10 E+00$ & $4.65 E+00$ & $2.74 E+00$ & $3.30 E+04$ & $2.98 E+04$ \\
\hline $\mathrm{Pu}-241$ & $4.81 \mathrm{E}-02$ & $1.14 E+03$ & $1.50 \mathrm{E}+01$ & $9.88 \mathrm{E}-04$ & $2.30 \mathrm{E}-18$ & $5.52 \mathrm{E}-102$ & $1.65 \mathrm{E}-206$ & $4.78 E+06$ & $9.63 E-15$ \\
\hline $\mathrm{Pu}-242$ & $1.86 \mathrm{E}-06$ & $2.61 \mathrm{E}-02$ & $2.61 \mathrm{E}-02$ & $2.61 \mathrm{E}-02$ & $2.60 \mathrm{E}-02$ & $2.58 \mathrm{E}-02$ & $2.56 \mathrm{E}-02$ & $1.09 \mathrm{E}+02$ & $1.09 E+02$ \\
\hline Am-241 & $1.60 \mathrm{E}-03$ & $2.66 E+01$ & $1.06 E+03$ & $7.58 E+02$ & $2.47 E+02$ & $4.08 \mathrm{E}-01$ & $1.35 \mathrm{E}-04$ & $1.11 \mathrm{E}+05$ & $1.04 E+06$ \\
\hline$A m-242 m$ & $4.92 E-03$ & $8.84 E-02$ & $5.68 \mathrm{E}-02$ & $2.12 \mathrm{E}-02$ & $6.80 \mathrm{E}-04$ & $1.96 \mathrm{E}-12$ & $4.15 \mathrm{E}-23$ & $3.70 E+02$ & $2.85 E+00$ \\
\hline Am-243 & $9.39 E-05$ & $2.56 \mathrm{E}-01$ & $2.54 \mathrm{E}-01$ & $2.49 E-01$ & 2.34E-01 & $1.60 \mathrm{E}-01$ & $1.00 \mathrm{E}-01$ & $1.07 E+03$ & $9.79 E+02$ \\
\hline$c m-242$ & $1.55 \mathrm{E}+00$ & $7.28 \mathrm{E}-02$ & $1.48 \mathrm{E}-62$ & $2.00 \mathrm{E}-197$ & $0.00 E+00$ & $0.00 E+00$ & $0.00 \mathrm{E}+00$ & $3.05 E+02$ & $0.00 E+00$ \\
\hline $\mathrm{Cm}-244$ & $3.83 E-02$ & $1.94 \mathrm{E}+01$ & $6.16 \mathrm{E}-01$ & $2.91 E-04$ & $6.63 E-16$ & $1.98 \mathrm{E}-82$ & $1.37 E-165$ & $8.11 E+04$ & $2.78 \mathrm{E}-12$ \\
\hline$C n-245$ & $8.15 E-05$ & $2.19 \mathrm{E}-03$ & $2.18 \mathrm{E}-03$ & $2.14 \mathrm{E}-03$ & $2.02 \mathrm{E}-03$ & $1.46 \mathrm{E}-03$ & $9.71 \mathrm{E}-04$ & $9.19 \mathrm{E}+00$ & $8.48 E+00$ \\
\hline $\mathrm{Cm}-246$ & 1.47E-04 & $4.68 \mathrm{E}-04$ & $4.62 \mathrm{E}-04$ & $4.49 \mathrm{E}-04$ & 4.05E-04 & $2.25 \mathrm{E}-04$ & $1.08 \mathrm{E}-04$ & $1.96 E+00$ & $1.70 \mathrm{E}+00$ \\
\hline \multicolumn{2}{|c|}{ other act. } & $6.20 \mathrm{E}-01$ & $6.20 \mathrm{E}-01$ & $6.20 \mathrm{E}-01$ & $6.20 \mathrm{E}-01$ & $6.20 \mathrm{E}-01$ & $6.20 \mathrm{E}-01$ & $2.60 E+03$ & $2.60 E+03$ \\
\hline others & & $6.20 \mathrm{E}+04$ & & & & & & $0.00 E+00$ & $0.00 E+00$ \\
\hline total & & $6.34 E+04$ & $1.42 E+03$ & $1.50 E+03$ & $1.73 E+03$ & $2.40 E+03$ & $4.73 E+03$ & $5.74 E+06$ & $7.26 \mathrm{E}+06$ \\
\hline
\end{tabular}


Table 13. Inventory of a B1-2 Package, 2 of 2

\begin{tabular}{|c|c|c|c|c|c|c|c|}
\hline Nuclide & Sp Activity & & g/pkg & at & year & & \\
\hline 4191 & $(\mathrm{Ci} / \mathrm{g})$ & 10 & 100 & 300 & 1000 & 5000 & 10000 \\
\hline$c-14$ & $4.46 \mathrm{E}+00$ & $5.36 \mathrm{E}+00$ & $5.30 \mathrm{E}+00$ & $5.17 E+00$ & $4.75 \mathrm{E}+00$ & $2.93 E+00$ & $1.60 \mathrm{E}+00$ \\
\hline $\mathrm{Ni}-59$ & $7.57 E+04$ & $1.20 \mathrm{E}-03$ & $1.20 \mathrm{E}-03$ & $1.20 E-03$ & $1.19 E-03$ & $1.15 \mathrm{E}-03$ & $1.09 E-03$ \\
\hline$z x-93$ & $2.51 E-03$ & $7.93 \mathrm{E}+02$ & $7.93 E+02$ & $7.93 E+02$ & $7.92 \mathrm{E}+02$ & $7.91 E+02$ & $7.89 E+02$ \\
\hline $\mathrm{mb}-94$ & $1.87 E-01$ & $1.02 E+02$ & $1.02 E+02$ & $1.01 E+02$ & $9.86 E+01$ & $8.60 E+01$ & $7.25 E+01$ \\
\hline Tc-99 & $1.70 \mathrm{E}-02$ & $9.73 \mathrm{E}-01$ & $9.73 \mathrm{E}-01$ & $9.73 E-01$ & $9.70 \mathrm{E}-01$ & $9.58 \mathrm{E}-01$ & $9.42 E-01$ \\
\hline $\mathrm{U}-234$ & $6.25 \mathrm{E}-03$ & $2.87 E+00$ & $2.87 \mathrm{E}+00$ & $2.87 E+00$ & $2.86 \mathrm{E}+00$ & $2.83 E+00$ & $2.79 E+00$ \\
\hline$U-235$ & $2.16 \mathrm{E}-06$ & $1.19 E+02$ & $1.19 E+02$ & $1.19 E+02$ & $1.19 \mathrm{E}+02$ & $1.19 E+02$ & $1.19 E+02$ \\
\hline $\mathrm{U}-236$ & $6.47 \mathrm{E}-05$ & $5.96 \mathrm{E}+01$ & $5.96 \mathrm{E}+01$ & $5.96 \mathrm{E}+01$ & $5.96 \mathrm{E}+01$ & $5.96 \mathrm{E}+01$ & $5.96 E+01$ \\
\hline $\mathrm{U}-238$ & $3.36 \mathrm{E}-07$ & $1.42 E+04$ & $1.42 \mathrm{E}+04$ & $1.42 E+04$ & $1.42 E+04$ & $1.42 \mathrm{E}+04$ & $1.42 E+04$ \\
\hline Th-230 & $2.02 E-01$ & $0.00 E+00$ & $1.01 \mathrm{E}-03$ & $1.05 \mathrm{E}-02$ & $1.19 E-01$ & $2.69 \mathrm{E}+00$ & $9.40 E+00$ \\
\hline $\mathrm{Ra}-226$ & $9.88 \mathrm{E}-01$ & $0.00 E+00$ & $6.17 \mathrm{E}-03$ & $2.01 E-01$ & $7.32 E+00$ & $5.93 E+02$ & $2.97 E+03$ \\
\hline$N p-237$ & $7.05 E-04$ & $6.73 E+00$ & $2.52 E+05$ & $8.40 E+05$ & $1.89 E+06$ & $2.40 E+06$ & $2.39 \mathrm{E}+06$ \\
\hline Pu-238 & $1.71 \mathrm{E}+01$ & $1.96 \mathrm{E}+00$ & $9.61 \mathrm{E}-01$ & $1.98 \mathrm{E}-01$ & $7.85 E-04$ & $1.48 \mathrm{E}-17$ & $1.04 E-34$ \\
\hline Pu-239 & $6.22 \mathrm{E}-02$ & $7.56 \mathrm{E}+01$ & $7.54 \mathrm{E}+01$ & $7.50 \mathrm{E}+01$ & $7.35 E+01$ & $6.55 E+01$ & $5.67 E+01$ \\
\hline $\mathrm{Pu}-240$ & 2.28E-01 & $3.46 \mathrm{E}+01$ & $3.42 E+01$ & $3.35 E+01$ & $3.11 E+01$ & $2.04 E+01$ & $1.20 E+01$ \\
\hline Pu-241 & $1.03 E+02$ & $1.11 E+01$ & $1.46 \mathrm{E}-01$ & $9.60 \mathrm{E}-06$ & $2.23 E-20$ & $5.36 \mathrm{E}-104$ & $1.60 \mathrm{E}-208$ \\
\hline$P u-242$ & $3.82 E-03$ & $6.83 E+00$ & $6.83 E+00$ & $6.83 E+00$ & $6.82 \mathrm{E}+00$ & $6.77 E+00$ & $6.70 E+00$ \\
\hline Am-241 & $3.43 E+00$ & $7.74 E+00$ & $3.09 E+02$ & $2.21 E+02$ & $7.21 E+01$ & $1.19 \mathrm{E}-01$ & $3.95 E-05$ \\
\hline$A m-242 m$ & $9.72 E+00$ & $9.09 E-03$ & $5.84 \mathrm{E}-03$ & $2.19 \mathrm{E}-03$ & $7.00 \mathrm{E}-05$ & $2.02 E-13$ & $4.27 E-24$ \\
\hline Am-243 & $1.99 E-01$ & $1.29 E+00$ & $1.28 \mathrm{E}+00$ & $1.25 \mathrm{E}+00$ & $1.17 E+00$ & $8.06 \mathrm{E}-01$ & 5.04E-01 \\
\hline$C m-242$ & $3.31 E+03$ & $2.20 E-05$ & $4.47 \mathrm{E}-66$ & $6.04 \mathrm{E}-201$ & $0.00 E+00$ & $0.00 E+00$ & $0.00 E+00$ \\
\hline $\mathrm{Cm}-244$ & $8.09 E+01$ & $2.39 E-01$ & $7.62 \mathrm{E}-03$ & $3.59 E-06$ & $8.20 \mathrm{E}-18$ & $2.44 E-84$ & $1.70 E-167$ \\
\hline$C m-245$ & $1.72 \mathrm{E}-01$ & $1.27 \mathrm{E}-02$ & $1.27 \mathrm{E}-02$ & $1.24 \mathrm{E}-02$ & $1.18 \mathrm{E}-02$ & $8.48 E-03$ & $5.64 \mathrm{E}-03$ \\
\hline$C m-246$ & $3.07 E-01$ & $1.53 E-03$ & $1.51 E-03$ & $1.46 \mathrm{E}-03$ & $1.32 E-03$ & $7.35 \mathrm{E}-04$ & $3.53 E-04$ \\
\hline
\end{tabular}


Table 14. Inventory of a B1-3 Package

\begin{tabular}{|c|c|c|c|c|c|c|c|}
\hline Nuclide & Lambda & & & Ci/pkg at yr & & & \\
\hline 5 & $(/ \mathrm{a})$ & 10 & 100 & 300 & 1000 & 5000 & 10000 \\
\hline I-129 & $4.41 \mathrm{E}-08$ & $3.97 \mathrm{E}+02$ & $3.97 \mathrm{E}+02$ & $3.97 \mathrm{E}+02$ & $3.97 \mathrm{E}+02$ & $3.97 \mathrm{E}+02$ & $3.97 \mathrm{E}+02$ \\
\hline TOTAL & & $3.97 \mathrm{E}+02$ & $3.97 \mathrm{E}+02$ & $3.97 \mathrm{E}+02$ & $3.97 \mathrm{E}+02$ & $3.97 \mathrm{E}+02$ & $3.97 \mathrm{E}+02$ \\
\hline & & & & & & & \\
\hline Nuclide & Sp Activity & & & $\mathrm{g} / \mathrm{pkg}$ at $\mathrm{yr}$ & & & stream total \\
\hline 5 & (Ci/g) & 10 & 100 & 300 & 1000 & & Ci at 10 a \\
\hline I-129 & $1.77 \mathrm{E}-04$ & $2.24 \mathrm{E}+06$ & $2.24 \mathrm{E}+06$ & $2.24 \mathrm{E}+06$ & $2.24 \mathrm{E}+06$ & & $1.98 \mathrm{E}+03$ \\
\hline TOTAL & & $2.24 \mathrm{E}+06$ & $2.24 \mathrm{E}+06$ & $2.24 \mathrm{E}+06$ & $2.24 \mathrm{E}+06$ & & \\
\hline
\end{tabular}


Table 15. Inventory of a B1-4 Package, 1 of 2

\begin{tabular}{|c|c|c|c|c|c|c|c|c|c|}
\hline Nuclide & Lambda & & $\mathrm{Cl} / \mathrm{pkg}$ & at & year & & & stream & total \\
\hline 24550 & $(/ a)$ & 10 & 100 & 300 & 1000 & 5000 & 10000 & $\mathrm{Ci}$ at 10 a & at 1000 \\
\hline$c-14$ & $1.21 \mathrm{E}-04$ & & $0.00 E+00$ & $0.00 E+00$ & $0.00 \mathrm{E}+00$ & $0.00 E+00$ & $0.00 \mathrm{E}+00$ & $0.00 \mathrm{E}+00$ & $0.00 E+00$ \\
\hline $\mathrm{Se}-79$ & $1.07 \mathrm{E}-05$ & $1.18 E+00$ & $1.18 E+00$ & $1.18 E+00$ & $1.17 E+00$ & $1.12 \mathrm{E}+00$ & $1.06 \mathrm{E}+00$ & $2.90 E+04$ & $2.87 E+04$ \\
\hline$S x-90$ & $2.43 \mathrm{E}-02$ & $1.63 E+05$ & $1.83 E+04$ & $1.41 E+02$ & $5.70 \mathrm{E}-06$ & $3.21 \mathrm{E}-48$ & $4.94 E-101$ & $4.01 E+09$ & $.40 \mathrm{E}-01$ \\
\hline$Y-90$ & $9.48 \mathrm{E}+01$ & $1.63 E+05$ & $0.00 \mathrm{E}+00$ & $0.00 \mathrm{E}+00$ & $0.00 E+00$ & $0.00 \mathrm{E}+00$ & $0.00 \mathrm{E}+00$ & $4.01 E+09$ & $0.00 E+00$ \\
\hline $2 x-93$ & $4.53 \mathrm{E}-07$ & $5.19 E+00$ & $5.19 \mathrm{E}+00$ & $5.19 E+00$ & $5.19 E+00$ & $5.18 \mathrm{E}+00$ & $5.17 \mathrm{E}+00$ & $1.27 E+05$ & $.27 E+05$ \\
\hline TC-99 & 3.29E-06 & $3: 36 E+01$ & $3.36 \mathrm{E}+01$ & $3.36 E+01$ & $3.35 E+01$ & $3.31 E+01$ & $3.25 E+01$ & +05 & $E+05$ \\
\hline Pd-107 & $.07 E-07$ & $3.20 \mathrm{E}-01$ & $3.20 \mathrm{E}-01$ & $3.20 E-01$ & -01 & -01 & & 03 & +03 \\
\hline Sn-126 & & +00 & +00 & .00 & & & & 24 & +04 \\
\hline Cs-135 & & 9. & 01 & & & & & & +04 \\
\hline Cs -137 & & & 04 & & 05 & 45 & 95 & +09 & -01 \\
\hline $\mathrm{sm}-$ & 7.7 & 8.6 & 02. & 01 & & 14 & 31 & +07 & +04 \\
\hline Eu-154 & 02 & 1.2 & 01 & 06 & 30 & 67 & 00 & +08 & -26 \\
\hline$u-234$ & -06 & 3.4 & -03 & -03 & 03 & 03 & 03 & +01 & $E+01$ \\
\hline$u-235$ & 9.7 & 4.9 & $=-05$ & -05 & -05 & -05 & -05 & $+\infty$ & +00 \\
\hline $\mathrm{U}-236$ & $2.96 \mathrm{E}-08$ & $7.43 \mathrm{E}-04$ & $7.43 \mathrm{E}-04$ & -04 & -04 & -04 & .04 & +01 & +01 \\
\hline$U-238$ & 1.55E-09 & $9.21 E-04$ & $9.21 \mathrm{E}-04$ & -04 & -04 & -04 & 04 & 01 & +01 \\
\hline $\mathrm{Th}-230$ & $9.00 \mathrm{E}-05$ & $0.00 E+00$ & E-05 & -04 & 03 & -01 & 01 & +00 & +02 \\
\hline $\mathrm{Ra}-226$ & .04 & 0.0 & .03 & 02 & 00 & +02 & 02 & +00 & +04 \\
\hline Np-237 & & & 01 & & & +02 & 02 & $E+01$ & $E+06$ \\
\hline$P u-2$ & & +00 & +00 & -01 & -03 & -17 & 34 & $E+05$ & $E+01$ \\
\hline Pu- & 2.8 & 9.0 & -01 & -01 & 01 & -01 & .01 & $2.23 E+04$ & $E+04$ \\
\hline Pu-2 & -04 & $1.52 E+00$ & $1.51 \mathrm{E}+00$ & $1.47 \mathrm{E}+00$ & $1.37 E+00$ & $8.97 \mathrm{E}-01$ & $E-01$ & $3.73 E+04$ & $E+04$ \\
\hline Pu-241 & $4.81 \mathrm{E}-02$ & $2.20 \mathrm{E}+02$ & $2.89 \mathrm{E}+00$ & $1.91 E-04$ & $4.43 E-19$ & $1.06 \mathrm{E}-102$ & $3.18 \mathrm{E}-207$ & $5.40 E+06$ & $E-14$ \\
\hline $\mathrm{Pu}-242$ & 1.86E-06 & $5.03 \mathrm{E}-03$ & $5.03 \mathrm{E}-03$ & 5.03E-03 & 5.02E-03 & $4.98 \mathrm{E}-03$ & $4.94 \mathrm{E}-03$ & $1.24 E+02$ & $1.23 \mathrm{E}+02$ \\
\hline$A m-241$ & $1.60 \mathrm{E}-03$ & $5.12 E+00$ & $2.04 E+02$ & $1.46 E+02$ & 4.77E+01 & 7.86E-02 & $.61 \mathrm{E}-05$ & $1.26 \mathrm{E}+05$ & $1.17 E+06$ \\
\hline$A m-242 m$ & 4.92E-03 & $1.70 \mathrm{E}-02$ & 1.09E-02 & $4.10 \mathrm{E}-03$ & $1.31 \mathrm{E}-04$ & $3.78 \mathrm{E}-13$ & $8.00 \mathrm{E}-24$ & $1.18 \mathrm{E}+02$ & $3.22 E+00$ \\
\hline$A m-243$ & 9.39E-05 & $4.94 E-02$ & $4.90 \mathrm{E}-02$ & $4.81 \mathrm{E}-02$ & $4.50 \mathrm{E}-02$ & $3.09 \mathrm{E}-02$ & $.93 \mathrm{E}-02$ & $E+03$ & +03 \\
\hline$C m-242$ & $1.55 \mathrm{E}+00$ & $1.40 \mathrm{E}-02$ & $2.86 \mathrm{E}-63$ & $3.85 \mathrm{E}-198$ & $0.00 E+00$ & $0.00 \mathrm{E}+00$ & 00 & +02 & $\underline{00}$ \\
\hline $\mathrm{Cm}-244$ & $3.83 E-02$ & $3.73 E+00$ & $1.19 \mathrm{E}-01$ & 5.61E-05 & $1.28 E-16$ & -83 & 66 & & \\
\hline $\mathrm{Cm}-245$ & $8.15 E-05$ & 4.23E-04 & $4.20 \mathrm{E}-04$ & $4.13 E-04$ & -04 & 04 & & $E+01$ & $E+00$ \\
\hline $\mathrm{Cm}-246$ & $1.47 \mathrm{E}-04$ & $9.03 \mathrm{E}-05$ & .05 & 05 & & -05 & 2.09E-05 & $2.22 E+00$ & $1.92 E+00$ \\
\hline$B a-137 m$ & 1.50 & $1.97 E+05$ & $0.00 \mathrm{E}+00$ & $0.00 E+00$ & $0.00 E+00$ & $0.00 E+00$ & $0.00 E+00$ & $E+09$ & $0.00 E+00$ \\
\hline total & & $7.72 E+05$ & $4.83 E+04$ & $8.29 E+02$ & $3.52 \mathrm{E}+02$ & $4.84 E+02$ & $9.34 E+02$ & $1.89 \mathrm{E}+10$ & $8.65 E+06$ \\
\hline
\end{tabular}


Table 15. Inventory of a B1-4 Package, 2 of 2

\begin{tabular}{|c|c|c|c|c|c|c|c|}
\hline Nuclide & Sp Activity & & g/pkg & at & year & & \\
\hline 24550 & $(\mathrm{Cl} / \mathrm{g})$ & 10 & 100 & 300 & 1000 & 5000 & 10000 \\
\hline$c-14$ & $4.46 \mathrm{E}+00$ & $0.00 E+00$ & $0.00 E+00$ & $0.00 E+00$ & $0.00 E+00$ & $0.00 \mathrm{E}+00$ & $0.00 E+00$ \\
\hline $5 e-79$ & $6.97 \mathrm{E}-02$ & $1.69 E+01$ & $1.69 E+01$ & $1.69 E+01$ & $1.68 \mathrm{E}+01$ & $1.61 E+01$ & $1.52 E+01$ \\
\hline $5 x-90$ & $1.36 E+02$ & $1.20 E+03$ & $1.34 E+02$ & $1.04 E+00$ & $4.19 E-08$ & $2.36 \mathrm{E}-50$ & $3.63 \mathrm{E}-103$ \\
\hline$Y-90$ & $E+05$ & $3.00 \mathrm{E}-01$ & $0.00 E+00$ & $0.00 \mathrm{E}+00$ & $0.00 E+00$ & $0.00 E+00$ & $00 \mathrm{E}+00$ \\
\hline $2 x-93$ & & $2.07 E+03$ & 2.07E+03 & $2.07 E+03$ & $2.07 E+03$ & +03 & $06 \mathrm{E}+03$ \\
\hline TC-99 & & 1. & 3 & 03 & $1.98 \mathrm{E}+03$ & +03 & \\
\hline$P d-107$ & & 6. & 02 & & 02 & +02 & \\
\hline $5 n-126$ & 02 & 7.85 & $7.85 E+01$ & & 01 & +01 & \\
\hline Cs-135 & 3 & 8.6 & 02 & & & & \\
\hline Cs-137 & 8. & 2.6 & 3. & +00 & 07 & & \\
\hline $\mathrm{sm}-151$ & 2. & 3.2 & 1. & +00 & -02 & 16 & 32 \\
\hline$E u-154$ & 2.7 & 4.71 & 2 & 09 & -33 & 9.4 & $=+\infty 0$ \\
\hline $\mathrm{v}-234$ & 0.3 & $5.5:$ & $5.54 \mathrm{E}-01$ & $5.53 \mathrm{E}-01$ & $5.52 \mathrm{E}-01$ & $5.46 \mathrm{E}-01$ & $5.38 E-01$ \\
\hline $\mathrm{U}-235$ & 06 & 2.3 & +01 & +01 & $2.30 \mathrm{E}+01$ & $2.30 E+01$ & $2.30 E+01$ \\
\hline $3-23$ & & +01 & +01 & 01 & +01 & +01 & +01 \\
\hline $\mathrm{J}-23$ & & & & & & & 3 \\
\hline Th-230 & 01 & 0.0 & -04 & & & & \\
\hline $\mathrm{Ra}-226$ & 01 & 0.0 & .03 & 02 & +00 & & \\
\hline Np-237 & 04 & 1.30 & 4.8 & +05 & +05 & 05 & \\
\hline Pu-238 & 01 & 3.7 & 1.8 & 3.82 & -04 & -18 & -35 \\
\hline Pu-239 & 6.221 & $1.46 E+01$ & $1.45 E+01$ & $1.45 E+01$ & $1.42 \mathrm{E}+01$ & $1.26 \mathrm{E}+01$ & $1.09 E+01$ \\
\hline$P u-240$ & -01 & $6.67 E+00$ & $6.60 E+00$ & $6.47 \mathrm{E}+00$ & $6.00 E+00$ & $3.94 E+00$ & $2.32 E+00$ \\
\hline Pu-241 & $1.03 E+02$ & $2.14 E+00$ & $2.81 \mathrm{E}-02$ & $1.85 E-06$ & $4.30 \mathrm{E}-21$ & $1.03 E-104$ & $3.09 \mathrm{E}-209$ \\
\hline Pu-242 & 03 & $1.32 E+00$ & $1.32 E+00$ & $1.32 E+00$ & $1.31 E+00$ & $1.30 \mathrm{E}+00$ & $E+00$ \\
\hline$A m-241$ & & 1.4 & r01 & +01 & +01 & -02 & -06 \\
\hline Am-2 & & & & & & & \\
\hline $\mathrm{Am}-243$ & 01 & $2.48 \mathrm{E}-01$ & & & & & \\
\hline$c m-242$ & $3.31 E+03$ & 4.24E-06 & 8.63E-67 & $1.16 \mathrm{E}-201$ & $0.00 E+00$ & & \\
\hline $\mathrm{cm}-244$ & 01 & $4.61 E-02$ & $1.47 \mathrm{E}-03$ & $6.93 \mathrm{E}-07$ & $1.58 E-18$ & E.85 & 3.28 \\
\hline$m-245$ & & $2.46 \mathrm{E}-03$ & 2.4 & $2.40 \mathrm{E}-03$ & $2.27 \mathrm{E}-03$ & $1.64 \mathrm{E}-03$ & .03 \\
\hline$c m-246$ & 3.07E-01 & 2.94E-04 & $2.90 E-04$ & 2.82E-04 & $2.55 \mathrm{E}-04$ & $1.42 E-04$ & $6.81 \mathrm{E}-0$ \\
\hline
\end{tabular}


Table 16. Inventory of the A1 Process

\begin{tabular}{|c|c|c|c|c|c|c|c|c|c|c|}
\hline Nuclide & Lambda & A1-1, 2 & A1-3 & A1-4 & A1-6 & A1-7 & Stored U & A1-B & Total & RF \\
\hline & (1a) & at 10 a & at 10 a & at 10 a & at 10 a & at $10 \mathrm{a}$ & at 10 a & at 10 a & at $10 \mathrm{a}$ & \\
\hline$c-14$ & $1.21 E-04$ & $1.00 E+05$ & & & & & & & $1.00 E+05$ & 1 \\
\hline $\mathrm{Ni}-59$ & $9.24 \mathrm{E}-06$ & $3.82 \mathrm{E}+05$ & & & & & & & $3.82 E+05$ & 1 \\
\hline Se-79 & $1.07 \mathrm{E}-05$ & & & & $2.57 E+04$ & & & $2.52 E+02$ & $2.60 E+04$ & 1 \\
\hline Sr-90 & $2.43 E-02$ & & & $3.54 E+09$ & & $3.59 E+07$ & & & $3.58 E+09$ & 1 \\
\hline$x-90$ & $9.48 E+01$ & & & $3.54 E+09$ & & $3.59 E+07$ & & & $3.58 E+09$ & 1 \\
\hline$z_{x}-93$ & $4.53 E-07$ & $8.34 \mathrm{E}+03$ & & & $1.13 E+05$ & & & $1.10 E+03$ & $1.23 E+05$ & 1 \\
\hline $\mathrm{Nb}-94$ & $3.41 \mathrm{E}-05$ & $8.01 E+04$ & & & & & & & $8.01 E+04$ & 1 \\
\hline$T c-99$ & $3.29 \mathrm{E}-06$ & $6.70 \mathrm{E}+01$ & & & $8.21 E+05$ & & & $7.90 E+03$ & $8.29 E+05$ & 1 \\
\hline $\mathrm{Pd}-107$ & $1.07 \mathrm{E}-07$ & & & & $7.06 E+03$ & & & $6.48 E+01$ & $7.13 E+03$ & 1 \\
\hline $5 n-126$ & $6.93 \mathrm{E}-06$ & & & & $4.88 E+04$ & & & $4.68 E+02$ & $4.93 E+04$ & 1 \\
\hline$I-129$ & $4.41 \mathrm{E}-08$ & & $1.98 E+03$ & & & & & & $1.98 E+03$ & 1 \\
\hline $\mathrm{Cs}-135$ & $3.01 \mathrm{E}-07$ & & & $2.15 E+04$ & & $2.30 \mathrm{E}+02$ & & & $2.17 E+04$ & 1 \\
\hline Cg-137 & 2.31E-02 & & & $5.09 E+09$ & & $5.15 E+07$ & & & $5.14 E+09$ & 1 \\
\hline $5 m-151$ & 7.70E-03 & & & & & & & $1.71 \mathrm{E}+07$ & $1.71 E+07$ & 1 \\
\hline Eu-154 & $7.88 \mathrm{E}-02$ & & & & & & & $2.51 E+08$ & $2.51 E+08$ & 1 \\
\hline $\mathrm{u}-234$ & $2.83 E-06$ & $7.20 E+01$ & & & $1.73 E+03$ & & $7.31 E+04$ & $7.02 E+01$ & $7.50 \mathrm{E}+04$ & 1 \\
\hline $\mathrm{U}-235$ & $9.76 \mathrm{E}-10$ & $1.23 \mathrm{E}+00$ & & & $2.96 E+01$ & & $1.06 \mathrm{E}+03$ & $1.20 E+00$ & $1.09 \mathrm{E}+03$ & 1 \\
\hline$u-236$ & $2.96 \mathrm{E}-08$ & $1.59 E+01$ & & & $3.83 E+02$ & & $1.57 E+04$ & $1.55 E+01$ & $1.61 E+04$ & 1 \\
\hline $\mathrm{u}-238$ & 1.55E-09 & $1.95 \mathrm{E}+01$ & & & $4.69 E+02$ & & $1.95 \mathrm{E}+04$ & $1.91 E+01$ & $2.00 E+04$ & 1 \\
\hline Np-237 & $3.24 \mathrm{E}-07$ & $1.95 E+01$ & & & & & & $1.91 E+01$ & $3.86 \mathrm{E}+01$ & 514 \\
\hline Pu-238 & $7.90 \mathrm{E}-03$ & $1.39 E+05$ & & & & & & $1.35 E+05$ & $2.74 E+05$ & 535 \\
\hline Pu-239 & $2.88 \mathrm{E}-05$ & $1.95 E+04$ & & & & & & $1.91 \mathrm{E}+04$ & $3.86 E+04$ & 511 \\
\hline $\mathrm{Pu}-240$ & $1.06 \mathrm{E}-04$ & $3.29 E+04$ & & & & & & $3.21 E+04$ & $6.50 \mathrm{E}+04$ & 505 \\
\hline Pu-241 & 4.81E-02 & $4.73 E+06$ & & & & & & $4.61 \mathrm{E}+06$ & $9.35 E+06$ & 523 \\
\hline $\mathrm{Pu}-242$ & $1.86 \mathrm{E}-06$ & $1.08 E+02$ & & & & & & $1.05 E+02$ & $2.13 E+02$ & 508 \\
\hline Am-241 & $1.60 \mathrm{E}-03$ & $1.13 E+05$ & & & & & & $1.10 E+05$ & $2.23 E+05$ & 476 \\
\hline$A m-242 m$ & $4.92 \mathrm{E}-03$ & $3.70 \mathrm{E}+02$ & & & & & & $3.61 E+02$ & $7.31 E+02$ & 597 \\
\hline Am-243 & $9.39 \mathrm{E}-05$ & $1.08 \mathrm{E}+03$ & & & & & & $1.05 E+03$ & $2.13 E+03$ & 505 \\
\hline$C m-242$ & $1.55 E+00$ & $3.04 E+02$ & & & & & & $2.96 \mathrm{E}+02$ & $5.99 E+02$ & 601 \\
\hline$c m-244$ & $3.83 \mathrm{E}-02$ & $8.23 E+04$ & & & & & & $8.02 E+04$ & $1.63 \mathrm{E}+05$ & 512 \\
\hline$c m-245$ & $8.15 E-05$ & $9.26 \mathrm{E}+00$ & & & & & & $9.03 E+00$ & $1.83 E+01$ & 503 \\
\hline$c m-246$ & $1.47 \mathrm{E}-04$ & $1.90 \mathrm{E}+00$ & & & & & & $1.86 E+00$ & $3.76 \mathrm{E}+00$ & 523 \\
\hline others & & $2.60 E+08$ & & $6.85 E+09$ & $1.35 E+08$ & $5.14 E+07$ & & $5.75 E+08$ & $7.87 E+09$ & \\
\hline TOTAL & & $2.66 \mathrm{E}+08$ & $1.98 E+03$ & $1.90 \mathrm{E}+10$ & $1.36 E+08$ & $1.75 E+08$ & $1.09 E+05$ & $8.48 E+08$ & $2.04 \mathrm{E}+10$ & 1 \\
\hline
\end{tabular}


Table 17. Inventory of the $A 3$ Process

\begin{tabular}{|c|c|c|c|c|c|c|c|}
\hline Nuclide & Lambda & A3-2, 5 & $A 3-2,5$ & A 3-4 & A3-4 & Total & Ci at yr \\
\hline & (/a) & at $10 \mathrm{a}$ & at $1000 \mathrm{a}$ & at $10 \mathrm{a}$ & at $1000 \mathrm{a}$ & 10 & 1000 \\
\hline $\mathrm{Ni}-59$ & $9.24 E-06$ & $1.84 \mathrm{E}+04$ & $1.82 E+04$ & & & $1.84 E+04$ & $1.82 E+04$ \\
\hline Se-79 & $1.07 \mathrm{E}-05$ & $2.10 \mathrm{E}+04$ & $2.08 E+04$ & & & $2.10 \mathrm{E}+04$ & $2.08 E+04$ \\
\hline $5 x-90$ & $2.43 E-02$ & $0.00 \mathrm{E}+00$ & $0.00 \mathrm{E}+00$ & $1.57 \mathrm{E}+09$ & $5.50 \mathrm{E}-02$ & $1.57 E+09$ & $5.50 \mathrm{E}-02$ \\
\hline$Y-90$ & $9.48 \mathrm{E}+01$ & & & $1.57 \mathrm{E}+09$ & $0.00 E+00$ & & \\
\hline $\mathrm{Mb}-94$ & $3.41 \mathrm{E}-05$ & $4.69 E+01$ & $4.53 E+01$ & & & $4.69 E+01$ & $4.53 E+01$ \\
\hline TC-99 & $3.29 \mathrm{E}-06$ & $7.22 E+05$ & $7.20 E+05$ & & & $7.22 E+05$ & $7.20 \mathrm{E}+05$ \\
\hline Pd-107 & $1.07 \mathrm{E}-07$ & $1.17 E+04$ & $1.17 E+04$ & & & $1.17 E+04$ & $1.17 E+04$ \\
\hline $5 n-126$ & $6.93 E-06$ & $8.85 E+04$ & $8.79 E+04$ & & & $8.85 E+04$ & $8.79 E+04$ \\
\hline$I-129$ & $4.41 \mathrm{E}-08$ & & & $2.53 E+03$ & $2.53 E+03$ & $2.53 E+03$ & $2.53 E+03$ \\
\hline Cs-135 & $3.01 \mathrm{E}-07$ & & & $8.74 E+04$ & $8.74 E+04$ & $8.74 E+04$ & $8.74 E+04$ \\
\hline Cs-137 & $2.31 \mathrm{E}-02$ & & & $4.16 \mathrm{E}+09$ & 4.84E-01 & $4.16 E+09$ & $4.84 \mathrm{E}-01$ \\
\hline Ba-137m & $1.50 \mathrm{E}+01$ & & & $3.93 E+09$ & $0.00 E+00$ & $3.93 E+09$ & $0.00 E+00$ \\
\hline $\mathrm{U}-234$ & $2.83 \mathrm{E}-06$ & $1.72 E+02$ & $1.72 E+02$ & & & $1.72 E+02$ & $1.72 E+02$ \\
\hline $0-235$ & $9.76 \mathrm{E}-10$ & $8.62 \mathrm{E}-02$ & 8.62E-02 & & & $8.62 \mathrm{E}-02$ & 8.62E-02 \\
\hline $\mathrm{U}-236$ & $2.96 \mathrm{E}-08$ & $1.03 E+00$ & $1.03 E+00$ & & & $1.03 E+00$ & $1.03 E+00$ \\
\hline $\mathrm{u}-238$ & $1.55 \mathrm{E}-09$ & $1.07 E+01$ & $1.07 E+01$ & & & $1.07 E+01$ & $1.07 E+01$ \\
\hline $\mathrm{Th}-230$ & $9.00 \mathrm{E}-05$ & $0.00 \mathrm{E}+00$ & $2.32 E+02$ & & & $0.00 E+00$ & $2.32 E+02$ \\
\hline Ra-226 & $4.33 \mathrm{E}-04$ & $0.00 \mathrm{E}+00$ & $6.95 E+04$ & & & $0.00 \mathrm{E}+00$ & $6.95 \mathrm{E}+04$ \\
\hline $\mathrm{N} p-237$ & $3.24 E-07$ & $3.94 E+01$ & $1.55 \mathrm{E}+07$ & & & $3.94 E+01$ & $1.55 E+07$ \\
\hline $\mathrm{Pu}-238$ & $7.90 \mathrm{E}-03$ & $1.72 E+06$ & $6.92 E+02$ & & & $1.72 E+06$ & $6.92 E+02$ \\
\hline$P u-239$ & $2.88 E-05$ & $2.34 \mathrm{E}+05$ & $2.27 E+05$ & & & $2.34 E+05$ & $2.27 E+05$ \\
\hline Pu-240 & $1.06 \mathrm{E}-04$ & $3.08 E+05$ & $2.77 E+05$ & & & $3.08 E+05$ & $2.77 E+05$ \\
\hline Pu-241 & $4.81 \mathrm{E}-02$ & $8.37 E+06$ & $1.69 \mathrm{E}-14$ & & & $8.37 E+06$ & $1.69 \mathrm{E}-14$ \\
\hline$P u-242$ & $1.86 \mathrm{E}-06$ & $5.91 E+02$ & $5.90 E+02$ & & & $5.91 E+02$ & $5.90 \mathrm{E}+02$ \\
\hline$A m-241$ & $1.60 \mathrm{E}-03$ & $5.42 E+05$ & $1.88 \mathrm{E}+06$ & & & $5.42 E+05$ & $1.88 \mathrm{E}+06$ \\
\hline$A m-242 m$ & $4.92 \mathrm{E}-03$ & $8.13 E+04$ & $6.26 \mathrm{E}+02$ & & & $8.13 E+04$ & $6.26 \mathrm{E}+02$ \\
\hline Am-243 & $9.39 z-05$ & $1.07 E+04$ & $9.76 \mathrm{E}+03$ & & & $1.07 E+04$ & $9.76 E+03$ \\
\hline$c m-242$ & $1.55 \mathrm{E}+00$ & $6.77 \mathrm{E}+04$ & $0.00 E+00$ & & & $6.77 \mathrm{E}+04$ & $0.00 \mathrm{E}+00$ \\
\hline$c m-244$ & $3.83 \mathrm{E}-02$ & $8.74 \mathrm{E}+05$ & $3.00 \mathrm{E}-11$ & & & $8.74 E+05$ & $3.00 \mathrm{E}-11$ \\
\hline$c m-245$ & $8.15 E-05$ & $5.66 \mathrm{E}+02$ & $5.22 E+02$ & & & $5.66 \mathrm{E}+02$ & $5.22 E+02$ \\
\hline$c m-246$ & $1.47 \mathrm{E}-04$ & $1.97 \mathrm{E}+02$ & $1.70 \mathrm{E}+02$ & & & $1.97 E+02$ & $1.70 E+02$ \\
\hline others & & & & $1.45 E+08$ & $0.00 E+00$ & $1.45 \mathrm{E}+08$ & $0.00 E+00$ \\
\hline TOTAL & & $1.31 E+07$ & $1.88 E+07$ & $1.14 E+10$ & $8.99 E+04$ & $1.14 E+10$ & $1.89 E+07$ \\
\hline
\end{tabular}


Table 18. Inventory for the B1 Process

\begin{tabular}{|c|c|c|c|c|c|c|c|c|c|}
\hline Nuclide & Bt-2 & B1-2 & B1-3 & B1-3 & Stored U & B1-4 & B1-4 & Total $\mathrm{Cl}$ & RF for \\
\hline & at $10 \mathrm{a}$ & at $1000 \mathrm{a}$ & at 10 a & at $1000 \mathrm{a}$ & at 10 a & at 10 a & at $1000 \mathrm{a}$ & at $10 a$ & $99.90 \%$ \\
\hline$c-14$ & $1.00 \mathrm{E}+05$ & 8.87E+04 & & & & & & $1.00 E+05$ & 1 \\
\hline $\mathrm{Ni}-59$ & $3.81 E+05$ & $3.77 E+05$ & & & & & & $3.81 E+05$ & 1 \\
\hline $\mathrm{Se}-79$ & & & & & & $2.90 E+04$ & $2.87 E+04$ & $2.90 E+04$ & 1 \\
\hline $5 x-90$ & & & & & & $4.01 E+09$ & $1.40 E-01$ & $4.01 E+09$ & 1 \\
\hline$Y-90$ & & & & & & $4.01 E+09$ & $0.00 E+00$ & $4.01 E+09$ & 1 \\
\hline $2 x-93$ & $8.34 E+03$ & $8.34 E+03$ & & & & $1.27 E+05$ & 1.27E+05 & $1.36 \mathrm{E}+05$ & 1 \\
\hline $\mathrm{Nb}-94$ & $8.00 E+04$ & $7.74 E+04$ & & & & & & $8.00 E+04$ & 1 \\
\hline Tc-99 & $6.92 E+01$ & $6.89 E+01$ & & & & $8.25 E+05$ & $8.23 E+05$ & $8.25 E+05$ & 1 \\
\hline$P d-107$ & & & & & & $7.86 E+03$ & $7.86 E+03$ & $7.86 \mathrm{E}+03$ & 1 \\
\hline $5 n-126$ & & & & & & $5.47 E+04$ & $5.44 \mathrm{E}+04$ & 5.47E+04 & 1 \\
\hline$I-129$ & & & $1.98 E+03$ & $1.98 \mathrm{E}+03$ & & & & $1.98 E+03$ & 1 \\
\hline $\mathrm{Cs}-135$ & & & & & & $2.43 E+04$ & $2.43 \mathrm{E}+04$ & $2.43 E+04$ & 1 \\
\hline Cs-137 & & & & & & $5.75 E+09$ & $6.70 \mathrm{E}-01$ & 5.75E+09 & 1 \\
\hline $5 m-151$ & & & & & & $2.13 E+07$ & $1.04 E+04$ & $2.13 E+07$ & 1 \\
\hline Eu-154 & & & & & & $3.12 E+08$ & $4.25 E-26$ & $3.12 E+08$ & 1 \\
\hline $\mathrm{u}-234$ & $7.52 \mathrm{E}+01$ & $7.50 E+01$ & & & $7.48 E+04$ & $8.49 E+01$ & $8.47 E+01$ & $7.50 E+04$ & 1 \\
\hline$U-235$ & $1.08 E+00$ & $1.08 E+00$ & & & $1.09 E+03$ & $1.22 E+00$ & $1.22 \mathrm{E}+00$ & $1.09 E+03$ & 1 \\
\hline $\mathrm{u}-236$ & $1.62 E+01$ & $1.62 E+01$ & & & $1.61 E+04$ & $1.83 E+01$ & $1.83 E+01$ & $1.61 E+04$ & 1 \\
\hline$u-238$ & $2.00 E+01$ & $2.00 E+01$ & & & $1.99 E+04$ & $2.26 \mathrm{E}+01$ & $2.26 \mathrm{E}+01$ & $2.00 E+04$ & 1 \\
\hline$T h-230$ & $0.00 E+00$ & $1.01 E+02$ & & & & $0.00 \mathrm{E}+00$ & $1.14 E+02$ & $0.00 E+00$ & \\
\hline $\mathrm{Ra}-226$ & $0.00 E+00$ & $3.03 E+04$ & & & & $0.00 \mathrm{E}+00$ & $3.42 E+04$ & $0.00 E+00$ & \\
\hline$N p-237$ & $1.99 \mathrm{E}+01$ & $5.59 E+06$ & & & & $2.25 E+01$ & $6.32 \mathrm{E}+06$ & $4.24 E+01$ & 469 \\
\hline $\mathrm{Pu}-238$ & $1.40 E+05$ & $5.63 E+01$ & & & & $1.59 \mathrm{E}+05$ & $6.36 \mathrm{E}+01$ & $2.99 E+05$ & 491 \\
\hline Pu-239 & $1.97 E+04$ & $1.92 E+04$ & & & & $2.23 E+04$ & $2.16 E+04$ & $4.20 E+04$ & 470 \\
\hline$P u-240$ & $3.30 \mathrm{E}+04$ & $2.98 E+04$ & & & & $3.73 E+04$ & $3.36 E+04$ & $7.03 E+04$ & 467 \\
\hline Pu-241 & $4.78 \mathrm{E}+06$ & $9.63 E-15$ & & & & $5.40 \mathrm{E}+06$ & $1.09 E-14$ & $1.02 \mathrm{E}+07$ & 480 \\
\hline$P u-242$ & $1.09 E+02$ & $1.09 E+02$ & & & & $1.24 \mathrm{E}+02$ & $1.23 E+02$ & $2.33 E+02$ & 465 \\
\hline$A m-241$ & $1.11 E+05$ & $1.04 E+06$ & & & & $1.26 \mathrm{E}+05$ & $1.17 E+06$ & $2.37 E+05$ & 449 \\
\hline$A m-242 m$ & $3.70 E+02$ & $2.85 E+00$ & & & & $4.18 \mathrm{E}+02$ & $3.22 \mathrm{E}+00$ & $7.89 E+02$ & 554 \\
\hline $\mathrm{Am}-243$ & $1.07 E+03$ & $9.79 E+02$ & & & & $1.21 \mathrm{E}+03$ & $1.11 E+03$ & $2.29 E+03$ & 471 \\
\hline$c m-242$ & $3.05 E+02$ & $0.00 E+00$ & & & & $3.45 \mathrm{E}+02$ & $0.00 \mathrm{E}+00$ & $6.50 \mathrm{E}+02$ & 555 \\
\hline $0 m-244$ & $8.11 E+04$ & $2.78 \mathrm{E}-12$ & & & & $9.16 \mathrm{E}+04$ & $3.14 \mathrm{E}-12$ & $1.73 E+05$ & 481 \\
\hline
\end{tabular}


Table 18. Inventory for the B1 Process

\begin{tabular}{|l|l|l|l|l|l|l|l|l|r|}
\hline $\mathrm{Cm}-245$ & $9.19 \mathrm{E}+00$ & $8.48 \mathrm{E}+00$ & & & & $1.04 \mathrm{E}+01$ & $9.57 \mathrm{E}+00$ & $1.96 \mathrm{E}+01$ & 470 \\
\hline $\mathrm{Cm}-246$ & $1.96 \mathrm{E}+00$ & $1.70 \mathrm{E}+00$ & & & & $2.22 \mathrm{E}+00$ & $1.92 \mathrm{E}+00$ & $4.18 \mathrm{E}+00$ & 470 \\
\hline others & $2.60 \mathrm{E}+03$ & $0.00 \mathrm{E}+00$ & & & & $2.93 \mathrm{E}+03$ & $0.00 \mathrm{E}+00$ & $5.53 \mathrm{E}+03$ & \\
\hline Ba-137m & & & & & & $4.84 \mathrm{E}+09$ & $0.00 \mathrm{E}+00$ & $4.84 \mathrm{E}+09$ & \\
\hline TOTAL & $5.74 \mathrm{E}+06$ & $7.26 \mathrm{E}+06$ & $1.98 \mathrm{E}+03$ & $1.98 \mathrm{E}+03$ & $1.12 \mathrm{E}+05$ & $1.89 \mathrm{E}+10$ & $8.65 \mathrm{E}+06$ & $1.89 \mathrm{E}+10$ & \\
\hline
\end{tabular}


that constitute less than 0.1 percent of the calculated total curie inventory at 1,000 years, the allowable annual release is a constant value, equal to $10^{-8}$ of the total curie inventory in the repository at 1,000 years. The release rate is input to total system performance calculations. Therefore it is necessary to calculate release rates for waste packages at Yucca Mountain.

We calculate release rates for the selected radionuclides using analytic solutions in Sadeghi et al. [1990] for the wet-drip bathtub water-contact mode. For the radionuclides, we consider the release of three types of species: solubility-limited species, species released congruent with solid-solid alteration of the waste matrix, and readily soluble species. In each case we give the release rates of the species as a function of time.

\subsection{The Wet-Drip Water-Contact Mode}

Here we refer to the dripping of water from overhead rock onto waste packages. This dripping may happen because of episodic fracture flow or a change in rock permeability may divert water into fractures that intersect the borehole. Drips are assumed to penetrate cracks in a failed container and to dissolve radionuclides as the radionuclide solution slowly rises in the container and finally overflows through other cracks and penetrations. Overflow of contaminated water is assumed to occur only near the top of the container. The contaminated water drips to the rock below. Water within the container is always well mixed from diffusion and thermal convection. We refer to this as the "wet-drip bathtub water-contact mode." We showed in Apted et al. [1991] that the release rates from the wet-drip bathtub water-contact mode are not very different from the wet-drip flow-through or the moist-continuous water-contact modes.

For details of calculations of release rates from LWR spent fuel (Scheme 1), see Sadeghi et al. [1991].

\subsection{Parameters Adopted for Calculating Release Rates}

The dimensions of waste containers have been given in Tables 1, 2 and 3.

\section{Hydrogeologic Conditions}

The far-field averaged flux at the emplacement horizon is taken to be $0.5 \mathrm{~mm} / \mathrm{a}$, which appears to be an upper bound for expected conditions [Barnard and Dockery 1991]. For the wet-drip water-contact mode we assume that water contact begins at 1000 years after emplacement.

\section{Release Mechanisms}

For the exotic waste matrix encountered in pyrochemical processing, Table 19 summarizes the release mechanisms for actinides and fission products. For the $U-234 \rightarrow T h-230 \rightarrow R a-226$ chain, the dissolution of the daughter nuclides are congruent with the mother nuclide, with zero initial inventory of the daughters assumed. 
Table 19. Release Mechanisms

\begin{tabular}{|c|c|c|c|c|c|}
\hline Matrix & None & AgI & Zeolite & Copper & Glass \\
\hline Actinides & $\begin{array}{c}\text { Solubility- } \\
\text { limited }\end{array}$ & NA & NA & $\begin{array}{c}\text { Solubility- } \\
\text { limited }\end{array}$ & $\begin{array}{c}\text { Solubility- } \\
\text { limited }\end{array}$ \\
\hline $\begin{array}{c}\text { Fission } \\
\text { Products }\end{array}$ & Instant & $\begin{array}{c}\text { Solubility- } \\
\text { limited }\end{array}$ & Instant & $\begin{array}{c}\text { Alteration- } \\
\text { controlled }\end{array}$ & $\begin{array}{c}\text { Alteration- } \\
\text { controlled }\end{array}$ \\
\hline
\end{tabular}

Table 20. Solubility Data

\begin{tabular}{|c|c|c|}
\hline \multicolumn{2}{|c|}{ Solubility $\left(\mathrm{g} / \mathrm{m}^{3}\right)$} \\
\hline Matrix & LWR SF \& Copper & Glass \\
\hline $\mathrm{Np}$ & $3.0 \times 10^{-4}$ & $9.4 \times 10^{-2}$ \\
\hline $\mathrm{Pu}$ & $9.5 \times 10^{-4}$ & $3.8 \times 10^{-8}$ \\
\hline $\mathrm{U}$ & 0.3 & $6 \times 10^{-2}$ \\
\hline $\mathrm{Am}$ & $3.8 \times 10^{-5}$ & $1.5 \times 10^{-3}$ \\
\hline Source & Wilson \& Bruton 1989 & Bruton 1988 \\
\hline
\end{tabular}

\section{Solubility}

For calculating the release rates of the solubility-limited species, the elemental solubility is needed. For solubilities of $\mathrm{U}, \mathrm{Np}, \mathrm{Pu}$ and Am dissolving from hardware and copper matrix, we use the concentrations of these elements measured in hot-cell leaching experiments of decladded LWR spent fuel [Wilson and Bruton 1989; Wilson 1990], shown in Table 20.

Solubilities of $\mathrm{U}, \mathrm{Np}, \mathrm{Pu}$ and Am dissolving from borosilicate glass have been calculated using the geochemical code EQ3/6 to simulate hot-cell leaching experiments of Wilson, also shown in Table 20 [Bruton 1988].

See Sadeghi et al. [1991] for a discussion of the uncertainties in these solubilities.

For AgI, the solubility was obtained from the commonly known solubility product constant [Burger, Scheek and Wiemers 1980].

\section{Matrix Alteration Rates}

For LWR spent fuel, we use an $\mathrm{UO}_{2}$ alteration rate of $10^{-3}$ per year from Wilson's leaching experiments [Wilson 1990].

For borosilicate glass, there is a slow corrosion reaction which releases fission products and actinides. From the experimental dissolution rate of lithium from borosilicate glass [Abrajano et al. 1988], the rate of reaction of the $\mathrm{SiO}_{2}$ glass matrix with water is $5.2 \mathrm{~g} / \mathrm{m}^{2}$-a. For a container with $1660 \mathrm{~kg}$ glass and assuming that the 
total reaction surface area, due to internal cracks, is 25 times the geometrical surface area $\left(0.27 \mathrm{~m}^{2}\right)$ [U. S. DOE 1987; Bruton 1988], the reaction rate would become $36 \mathrm{~g} / \mathrm{a}$. This results in a fractional alteration rate of $2 \times 10^{-5} / \mathrm{a}$.

Several of the reprocessing waste containers have copper matrix. Elemental copper is not stable in the oxidizing environment at Yucca Mountain. To estimate copper corrosion rate, we used data from a 16-year corrosion damage study of copper alloy in aqueous environments in the tropics, conducted by the U.S. Naval Research Laboratory [Southwell, Bultman and Alexander 1976]. In these tests, samples exposed to intermittent immersion in Pacific Ocean water and complete immersion in soft-water lake water resulted in the same corrosion rate. Over 16 years the average weight loss was $5 \mathrm{~g} / \mathrm{m}^{2}-\mathrm{a}$. We use this corrosion rate, in the form of a fractional alteration rate per year, for copper-matrix waste containers.

\subsection{Calculated Release Rates}

Release rates have been calculated for most of the 33 species that we decided to track as inventory. However, in this report we shall present only selected release rates.

In a parallel study, Hirschfelder et al. [1991] showed that these species are the only ones that will reach the water table and have the potential to reach the accessible environment.

All calculated release rates are listed in Appendix A. For the release rate of each species from a particular waste, the fractional release rate as well as the release rate in curie/annum are available. All these files are available from the authors via electronic mail or magnetic diskette.

The release rates in Figures 5 to 15 are for individual waste packages.

Figure 5 shows the fractional release rates of selected species from waste package A3-2,5 which results from pyrochemical processing of ALMR fuel. It contains electro-refining metal waste, with ALMR fuel hardware melted into the copper matrix. The actinides all have low fractional release rates because they are solubility limited. The highest fractional release rate is that of $\mathrm{Tc}-99$, where the fractional release rate approaches the copper alteration rate of $6.5 \times 10^{-5}$ per year. The release rate of $\mathrm{Pu}$ is split between the three isotopes present, $\mathrm{Pu}-239, \mathrm{Pu}-240$, and $\mathrm{Pu}-242$. As the shorter half-life $\mathrm{Pu}-240$ decays, the fractional release rate of Pu-242 increases because it now constitutes a bigger share of the elemental inventory.

Figure 6 shows the fractional release rates of selected species from waste package B1-4 which results from aqueous processing of LWR spent fuel. In contrast to the waste considered in Figure 1, this waste package, glass waste, contains both fission products and actinides. The fractional release rates of the fission products are essentially identical, and differ at later times due to the difference in decay rates. The high fractional release rates of americium and neptunium are due to the solubility used [Sadeghi et al. 1991].

Figures 7 and 8 show the release of Tc-99, as fractional release rates and as curie/annum, from all reprocessing 


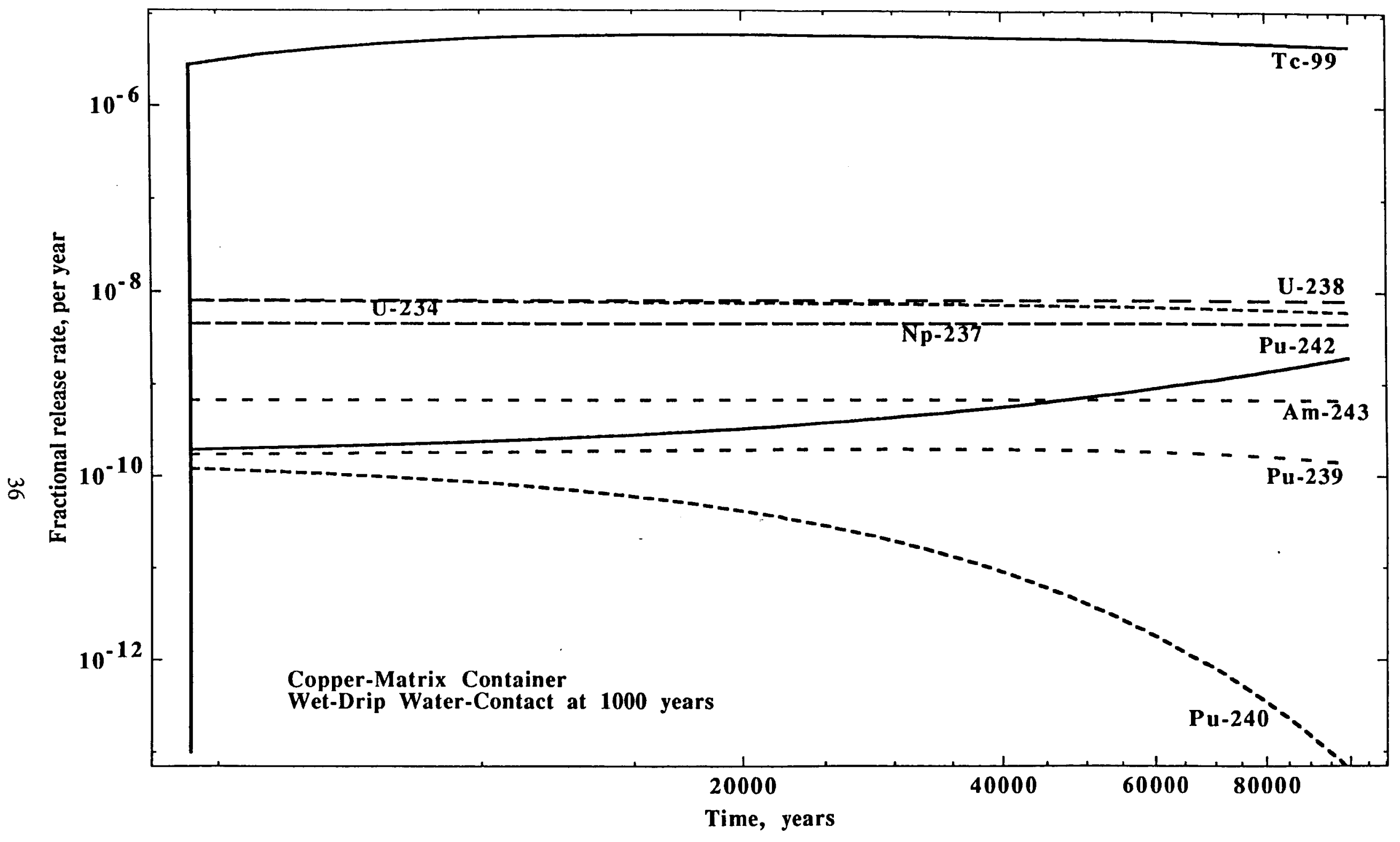

Figure 5. Fractional release rates of selected species from waste package $\mathbf{A 3 - 2 , 5}$ 


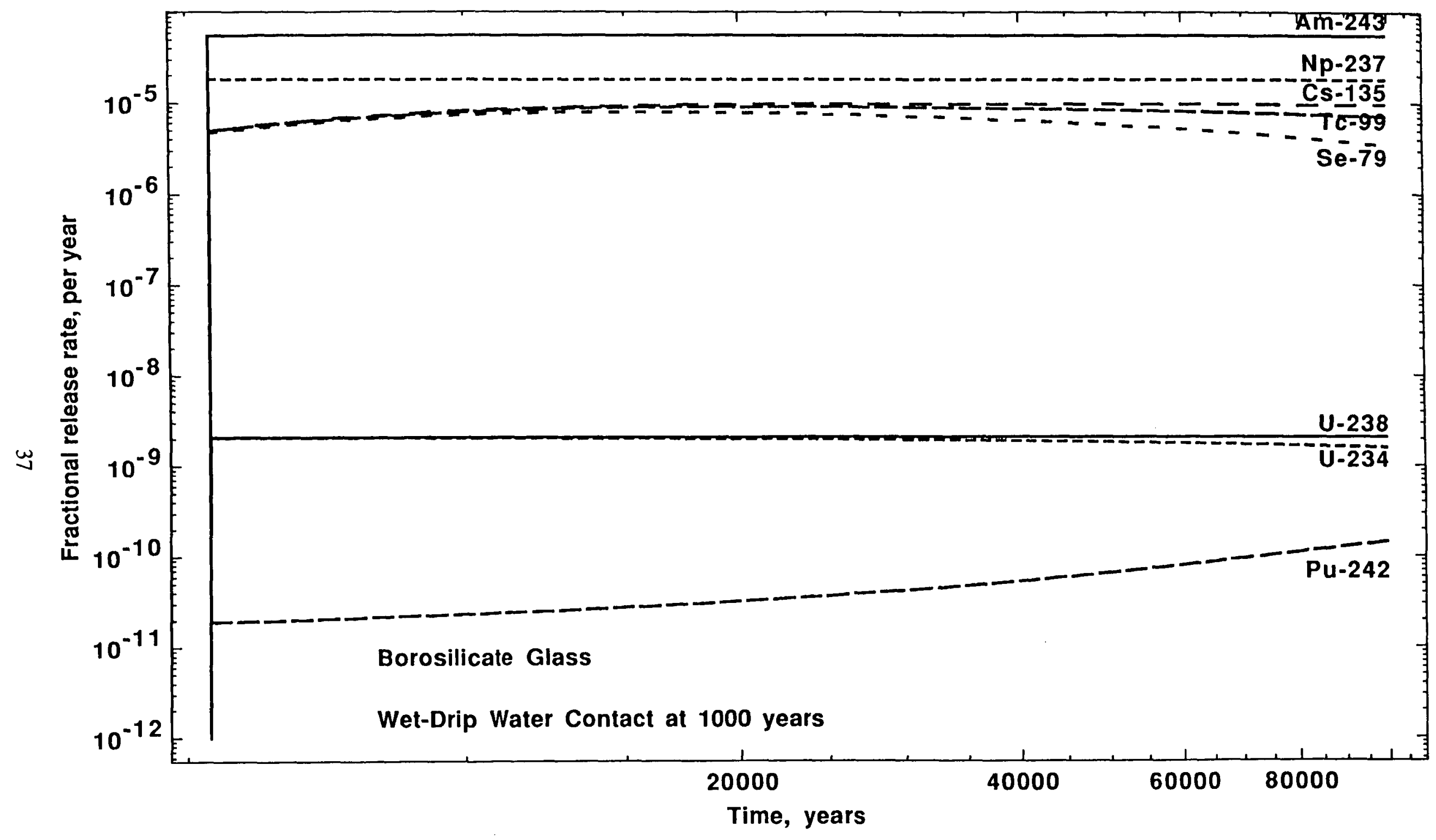

Figure 6. Fractional release rates of selected species from waste package B1-4 


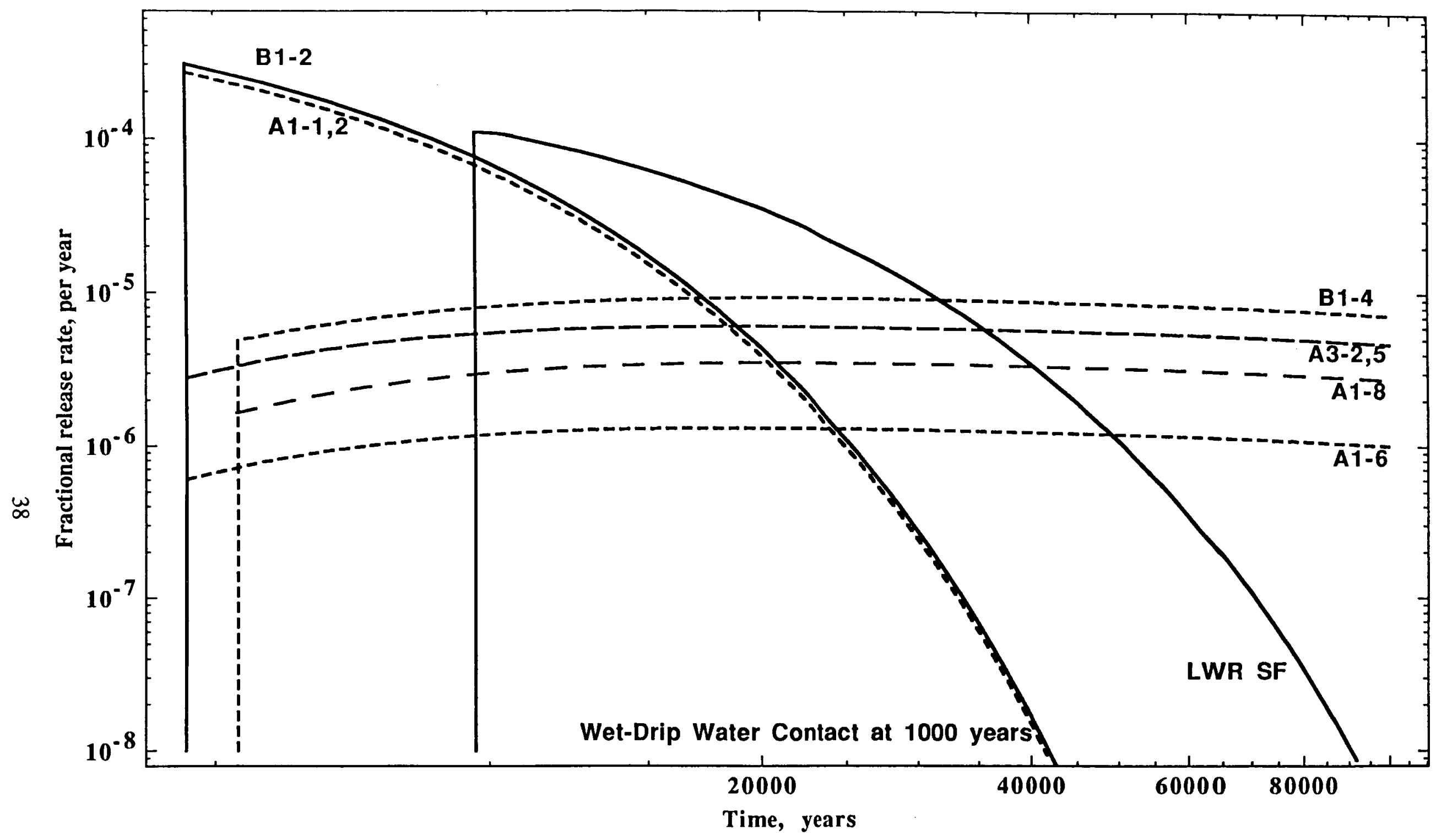

Figure 7. Fractional release rates of TC-99 from LWR spent fuel and reprocessed wastes 


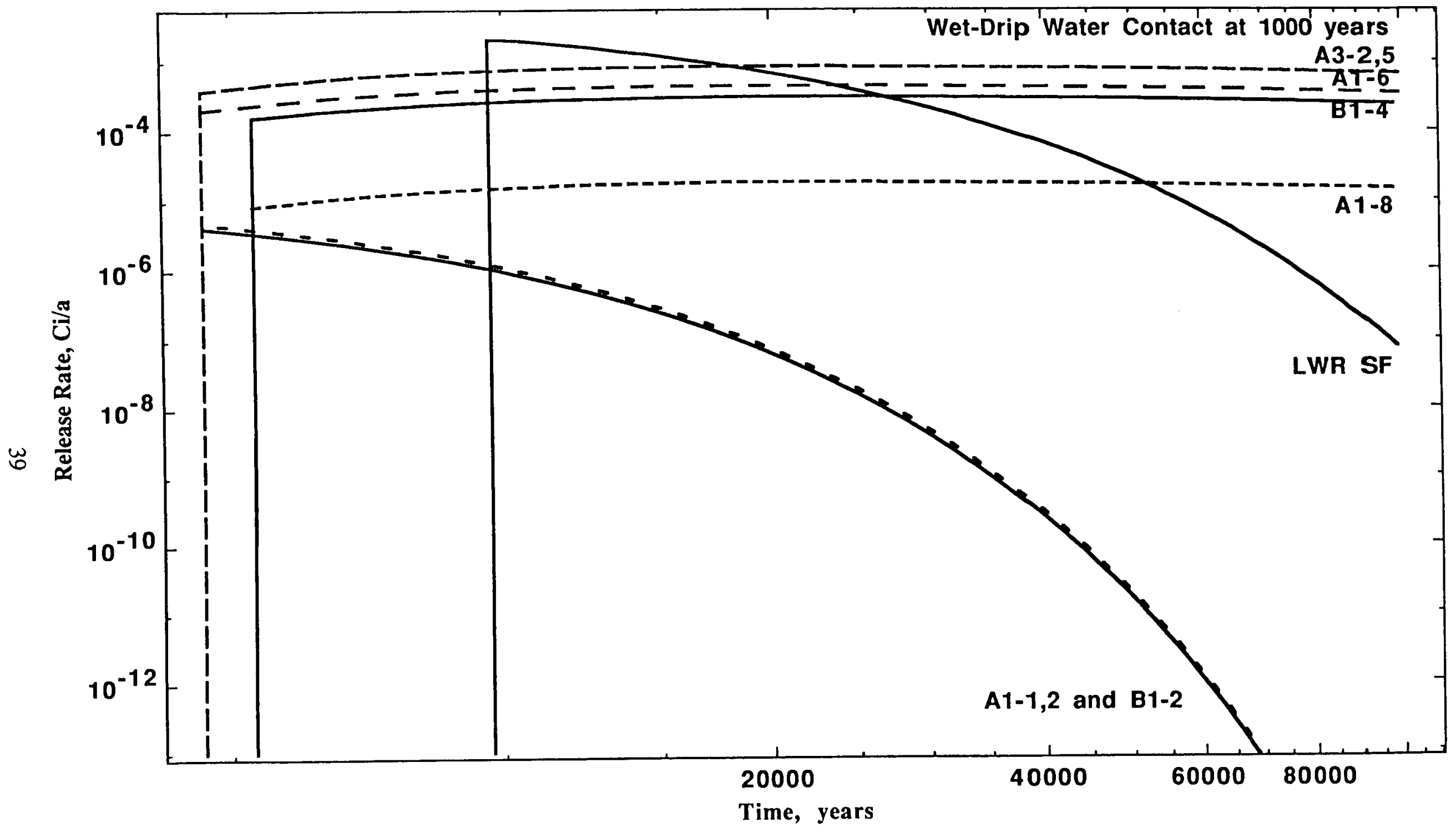

Figure 8. Release rates of TC-99 from LWR spent fuel and reprocessed wastes 
wastes, as well as from LWR spent fuel [Sadeghi et al. 1991]. The releases of Tc-99 from individual packages of reprocessed wastes are about the same as for LWR spent fuel. Because of the smaller void volume in the waste containers, releases begin sooner, and because of the slow matrix alteration rate in the copper-matrix wastes, the release rates continue at a high level for a longer period of time.

Figures 9 and 10 show the release of Cs-135, as fractional release rates and as curie/annum, from all reprocessing wastes, as well as from LWR spent fuel [Sadeghi et al. 1991]. The release rates of Cs-135 from reprocessed packages are generally lower than for LWR spent fuel, but the fractional release rates of several reprocessed packages are above the USNRC limit of $5 \times 10^{-5}$ for Cs-135.

Figure 11 shows the fractional release rates of I-129 from LWR spent fuel and from reprocessed waste. In A1-3 the waste form is the AgI compound. Because the allowable USNRC limit for the engineered barrier system is $5 \times 10^{-4}$ for I-129, this limit is not exceeded by any waste form.

Figure 12 shows the fractional release rates of $\mathrm{Np}-237$ from reprocessed wastes. The release of $\mathrm{Np}-237$ is solubility limited in all cases. Except for borosilicate glass, where $\mathrm{Np}-237$ has a high solubility, the release rates are very low.

Figure 13 shows the fractional release rates of U-238 and U-234 from reprocessed wastes. In these calculations these two species have been considered mixtures, rather than members of a decay chain. The releases of uranium species are solubility limited in all cases. The only difference between the two species is the shorter half life of $\mathrm{U}-234$. The fractional release rate is highest in the hardware package B1-2, while all other release rates are very low.

Figures 14 and 15 show the release of plutonium species, as fractional release rates and as curie/annum, from all reprocessing wastes. The release rate of $\mathrm{Pu}$ is partitioned into the three longest-lived isotopes, and that partition is shown for only one waste stream, A1-8. The release rate of only Pu-242, the longest-lived isotope, is shown from the other waste packages. Because Pu is solubility limited, all release rates are low.

We now calculate the aggregate release from entire repositories, represented by the schemes in Figure 1 . An equal amount of nuclides released from either scheme should result in the same dose at the point of discharge. Once radionuclides are released from waste, the buffering capacity of the rock controls the chemical form of the species, and its transport properties.

We multipliy the release rates of key radionuclides from the individual waste packages by the number of waste packages, and compare the overall release rate of that species from the two schemes, LWR SF vs reprocessing, for I-129, Tc-99, Np-237 and Pu isotopes. Figure 16 shows the release rate of I-129 from LWR spent fuel and reprocessed wastes. The peak release rate of I-129 from reprocessed wastes is approximately the same as that from LWR spent fuel, but starts earlier. For reprocessing wastes from both Schemes $2 a$ and $2 \mathrm{~b}$, most of the releases are instant release from the zeolite waste in A3-4. The solubility-limited release 


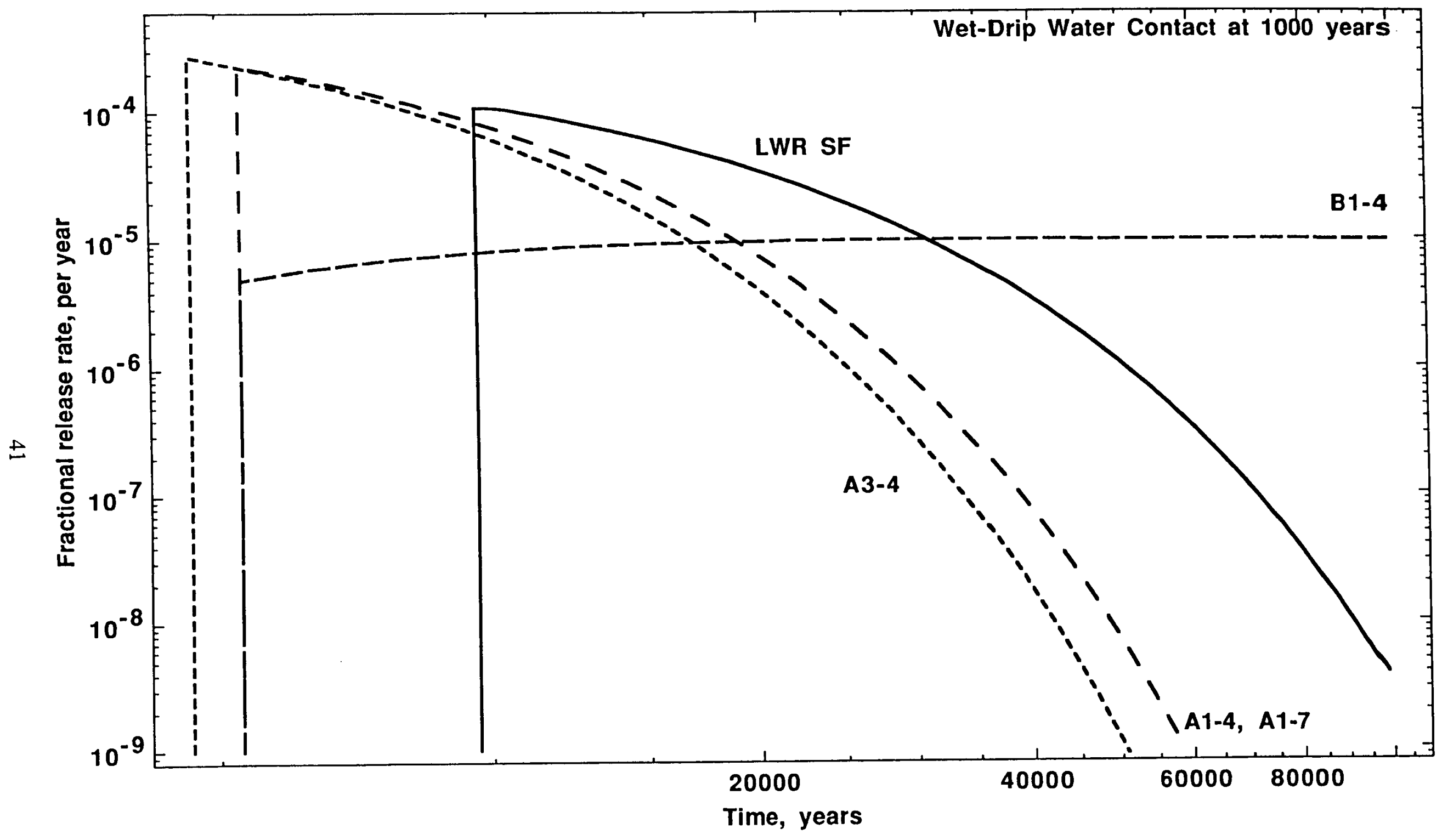

Figure 9. Fractional release rates of Cs-135 from LWR spent fuel and reprocessed wastes 


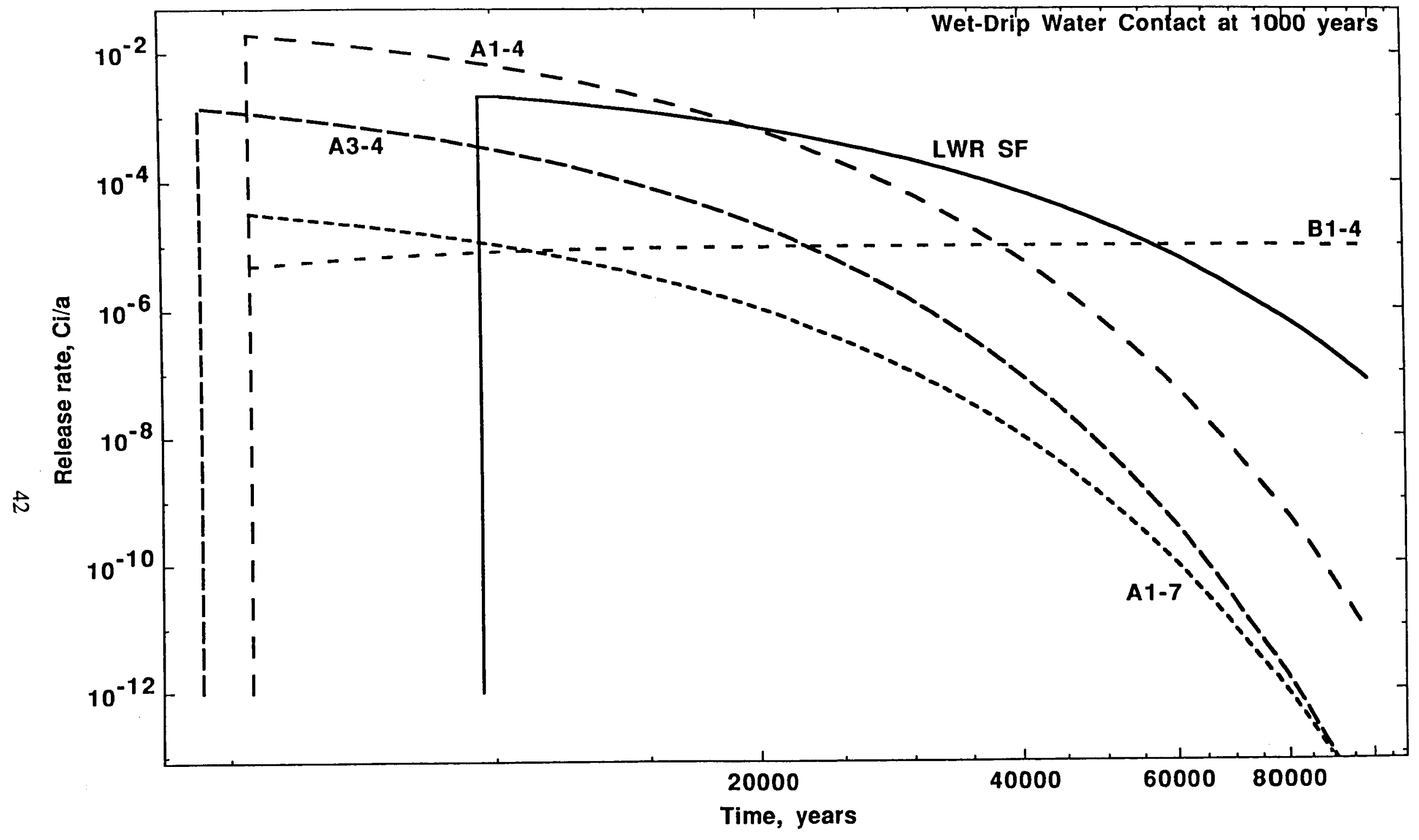

Figure 10. Release rates of Cs-135 from LWR spent fuel and reprocessed wastes 


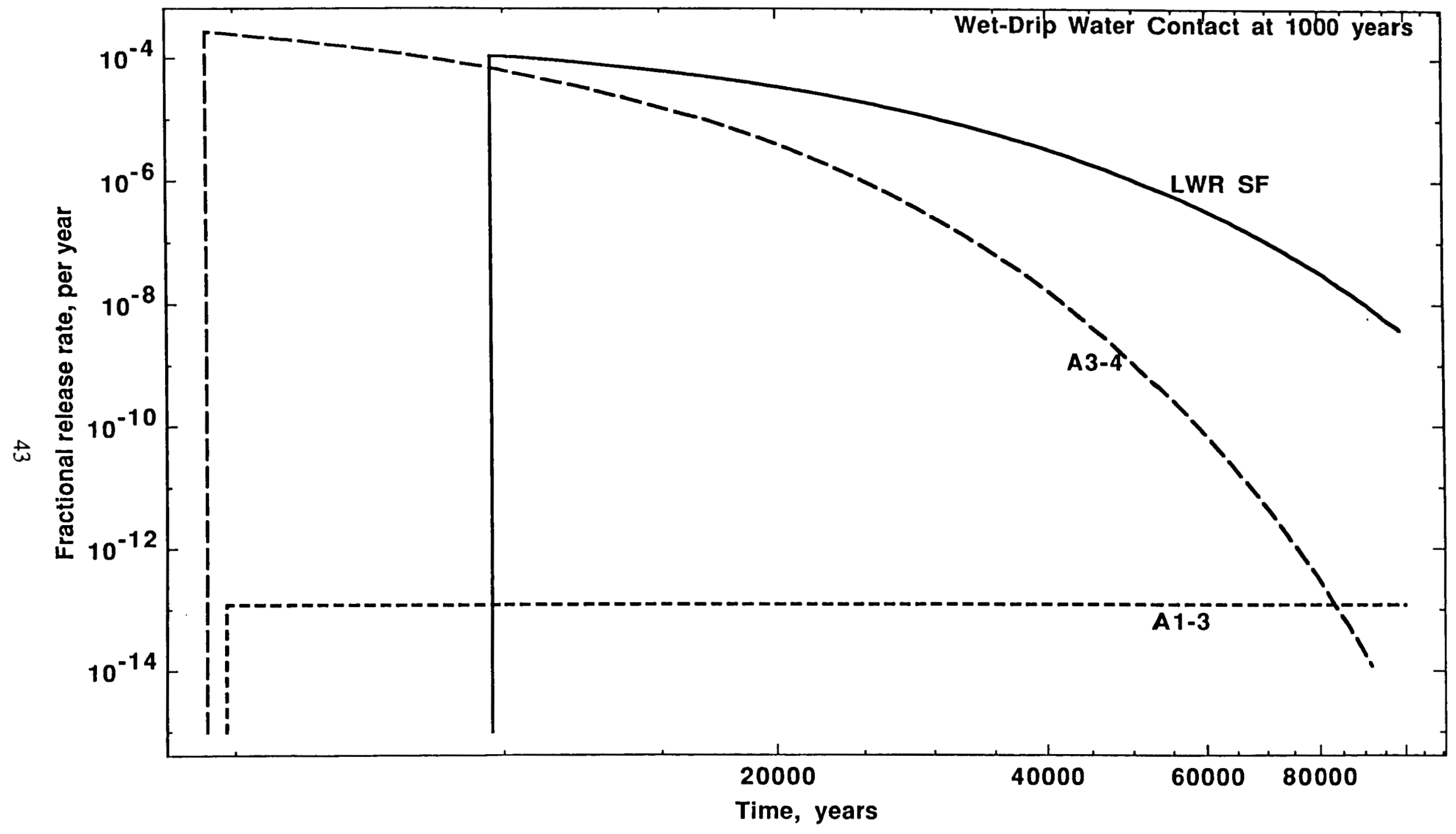

Figure 11. Fractional release rates of I-129 from LWR spent fuel and reprocessed wastes 


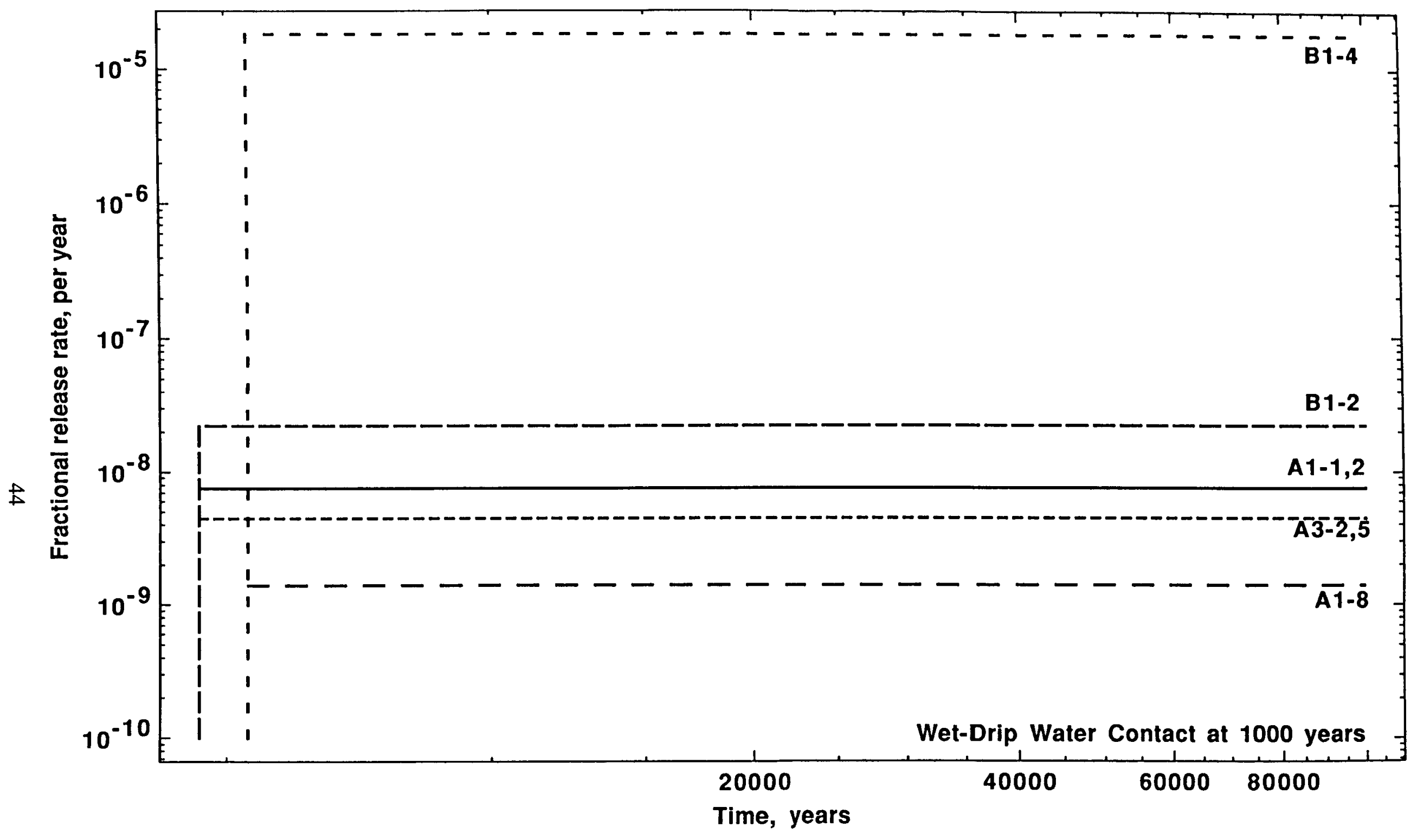

Figure 12. Fractional release rates of Np-237 from reprocessed wastes 


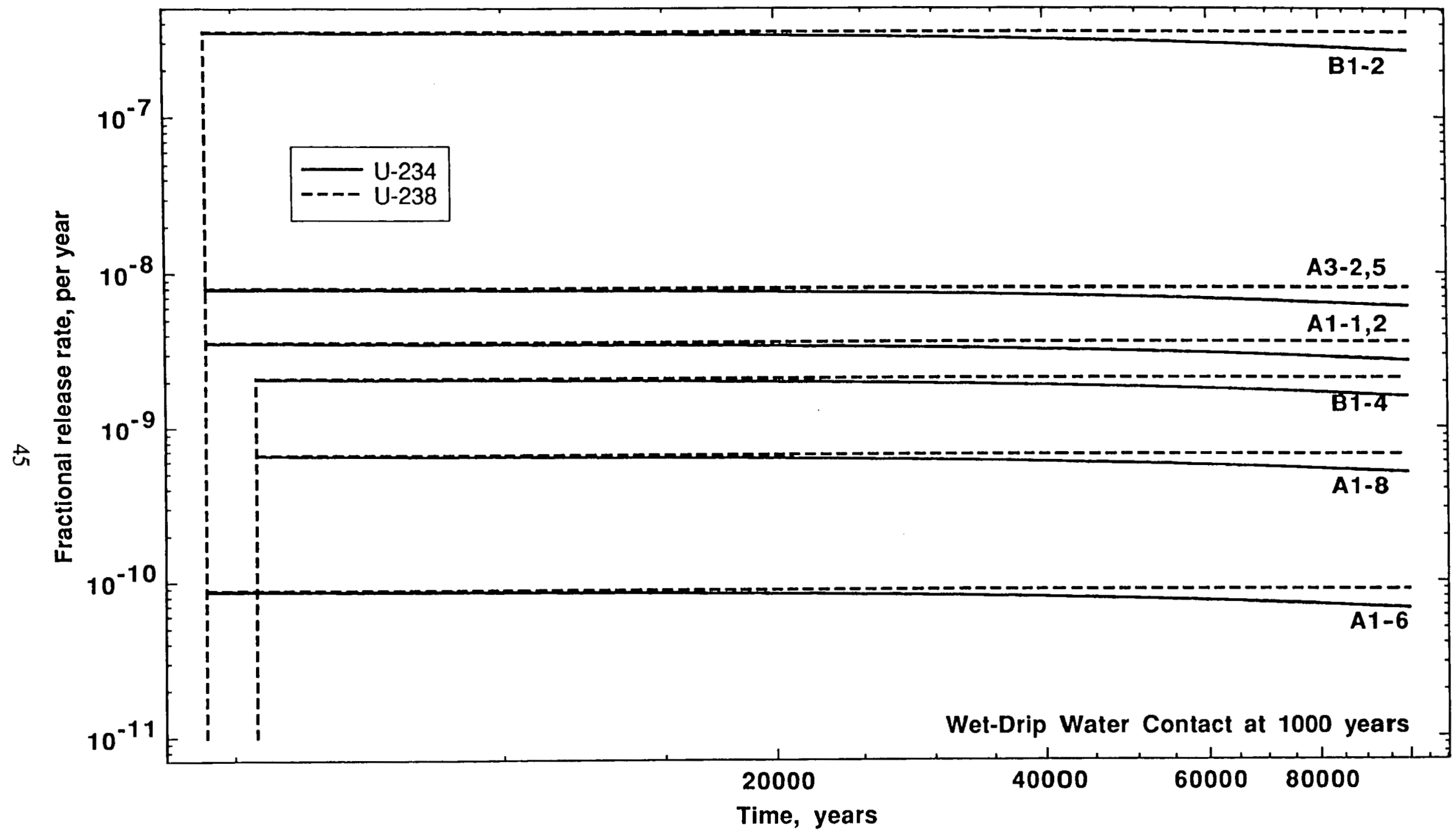

Figure 13. Fractional release rates of uranium species from LWR spent fuel and reprocessed wastes 


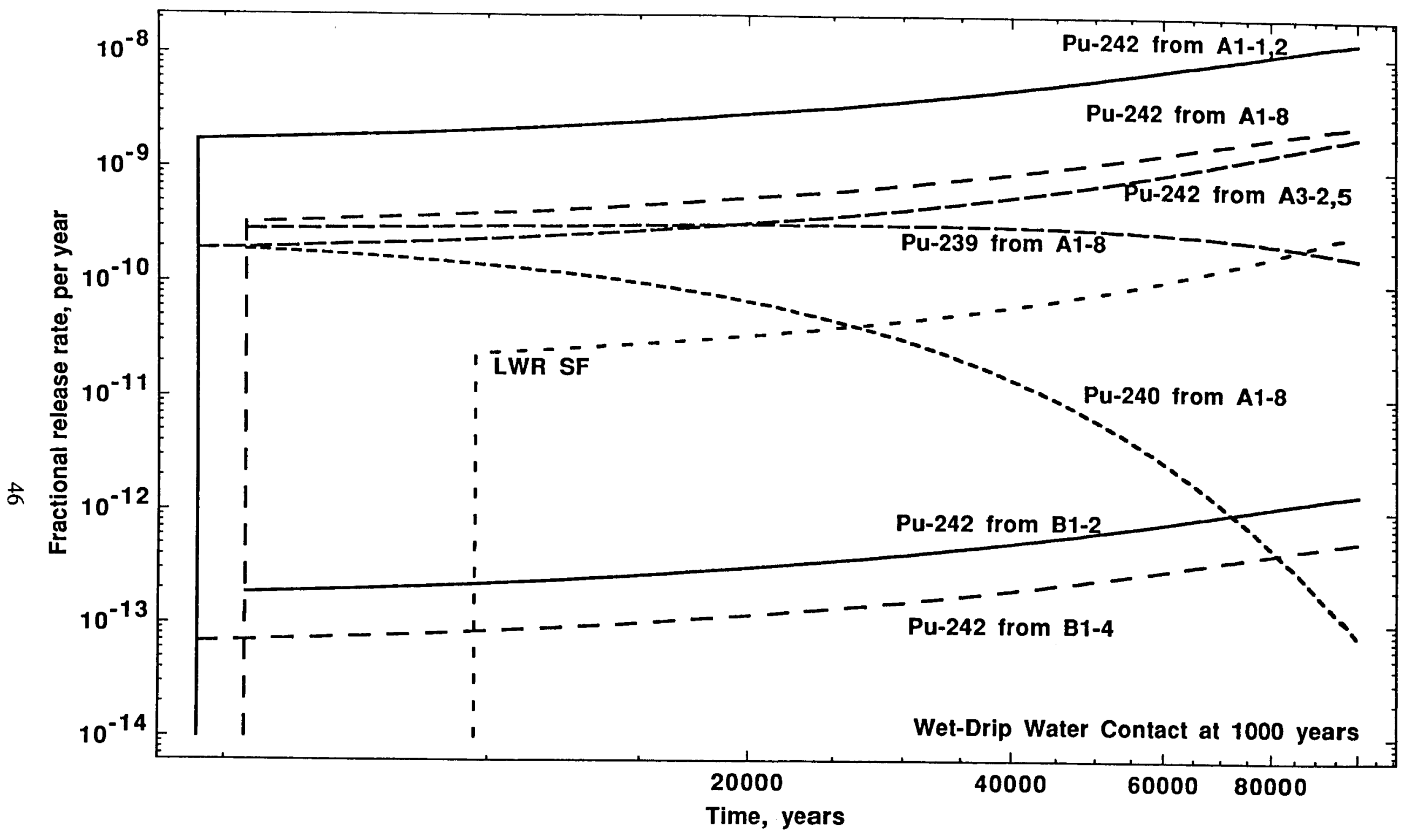

Figure 14. Fractional release rates of plutonium irom LWR spent fuel and reprocessed wastes 


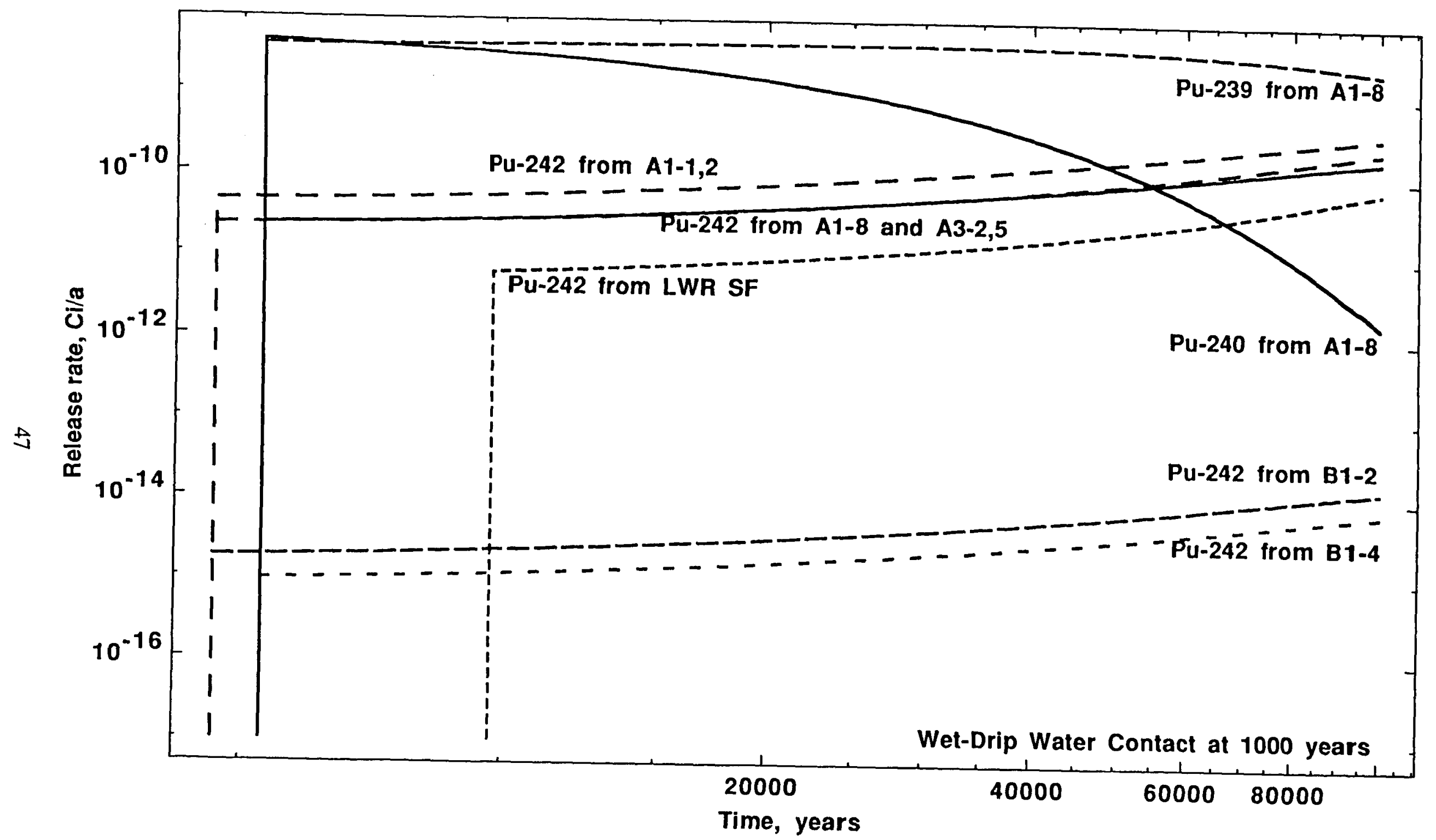

Figure 15. Release rates of plutonium from LWR spent fuel and reprocessed wastes 


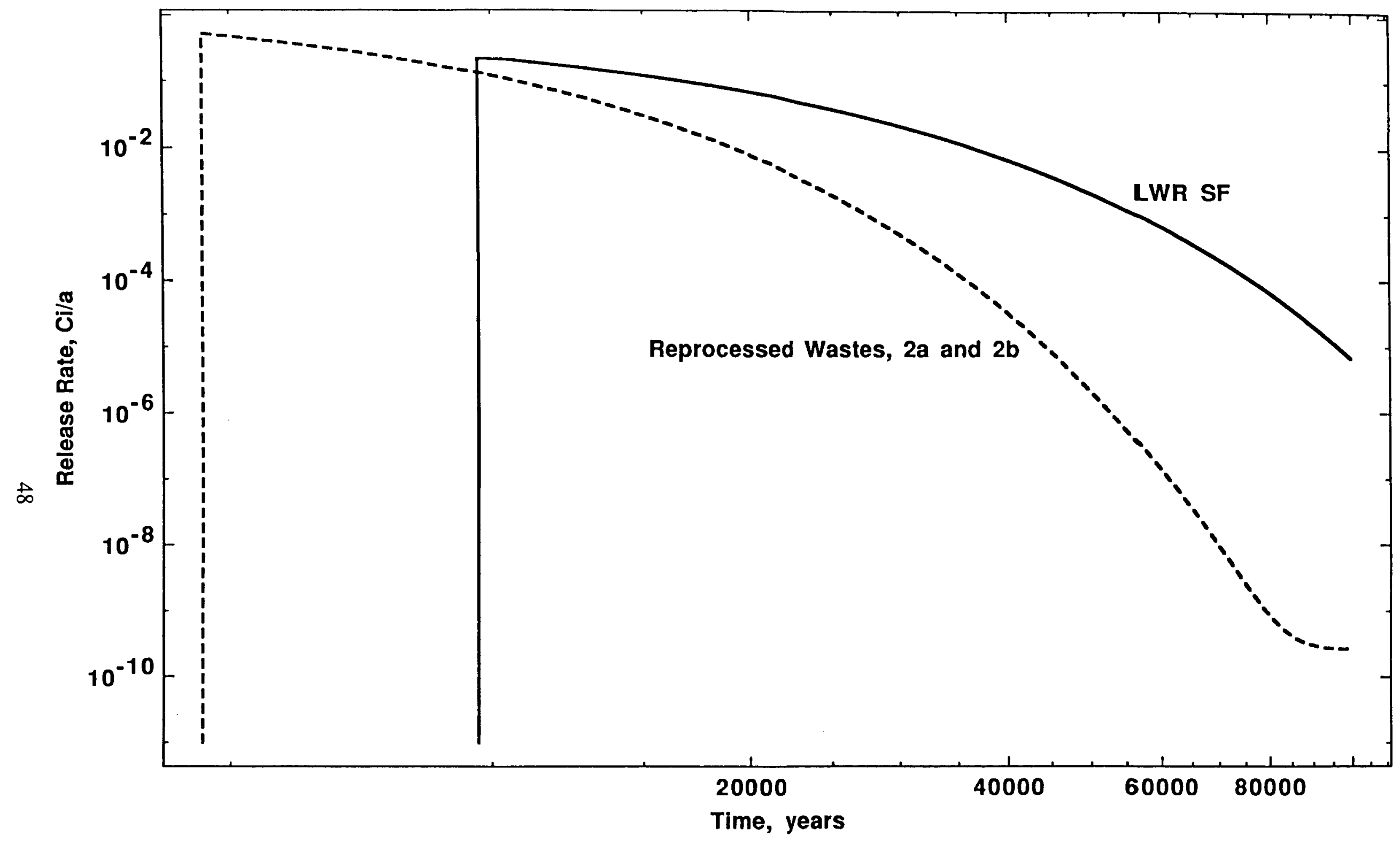

Figure 16. Release rates of $1-129$ from Schemes $1,2 a$ and $2 b$ 
from AgI from gaseous I-129 does not appear until about 80,000 years, in the form of a tail.

Figure 17 shows that for Tc-99 the peak release rate from LWR SF is higher by about a factor of 10 , but the releases from reprocessed wastes starts earlier and stays at a high level for a much longer time. This is because the LWR spent fuel waste container has a larger void volume, and release starts much later, and the alteration rate of LWR spent fuel is about two-orders of magnitude faster than the copper-matrix waste containers resulting from reprocessing.

For solubility-limited Np-237, Figure 18 shows that the release rate from LWR spent fuel is between that of Scheme $2 a$ and Scheme $2 b$. Within the uncertainty of our parameter values, we can say that the release rates of $\mathrm{Np}-237$ from LWR spent fuel and reprocessed wastes are equal.

Figure 19 shows the composite release rate of the plutonium isotopes from LWV spent fuel and reprocessing wastes. The combined release from LWR spent fuel is usually higher, but within a factor of 10 . Within the accuracy of the parameter values, these release rates can be considered equal.

The release rates in Figures 5 through 8 assumes that all waste packages begin water contact at 1000 years, and no credit has been taken for the time-distributed nature of package failure.

\section{Conclusions}

This report attempts to provide some of the basis for evaluating the benefits for waste disposal of partitioning and transmutation. Inventories of exotic waste packages are given. Release rates, for the wet-drip watercontact mode relevant to Yucca Mountain, have been calculated. For key radionuclides that are likely to reach the accessible environment, the release rates from reprocessed waste packages are shown to be approximately the same as the release rate from LWR spent fuel. If the release radionuclides behave similarly during transport, then it would appear that the releases from reprocessed waste packages are similar to LWR spent fuel.

Several caveats are in order about the results presented here. While we use the same methodology for calculating release rates as for the potential repository at Yucca Mountain, in calculating release rates for Yucca Mountain we use well established solubilities. In this study we assumed that solubilities for spent fuel and borosilicate glass dissolution are applicable, a step that has to be justified by experiments.

We also assumed, quite arbitarily, that water contact begins at 1000 years. For spent fuel disposal at Yucca Mountain, extensive thermal studies showed that re-condensation can begin at about that time. For the exotic wastes from partitioning and transmutation, we do not know whether this is true.

Given the validity of these assumptions, actinide-burning appears to offer no benefit for waste disposal, in terms of radionuclide releases from a geologic repository. Our conclusion collaborates similar studies in other countries [Priem, Bretheau and Cernes 1990]. 


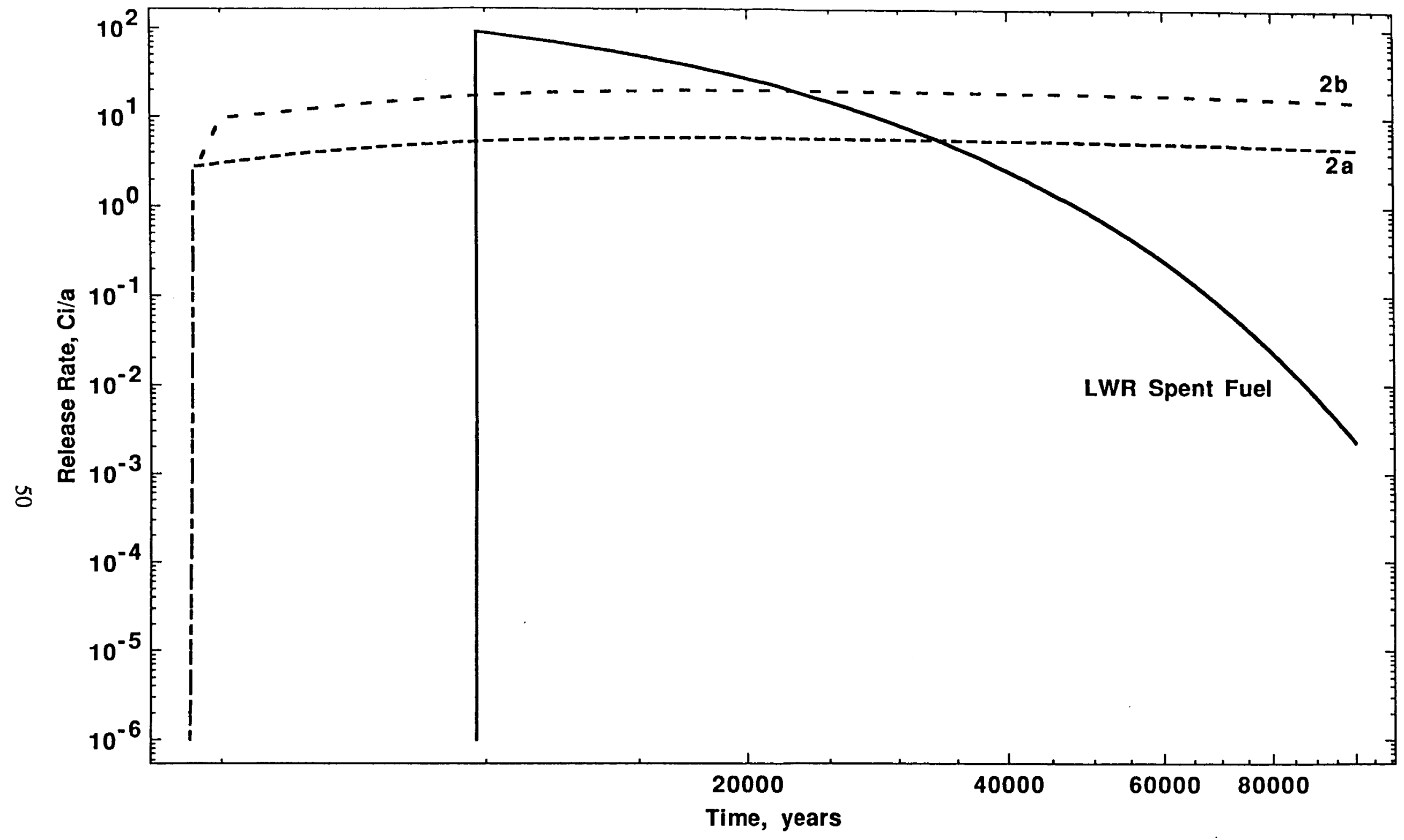

Figure 17. Release rates of TC-99 from Schemes $1,2 a$ and $2 b$ 


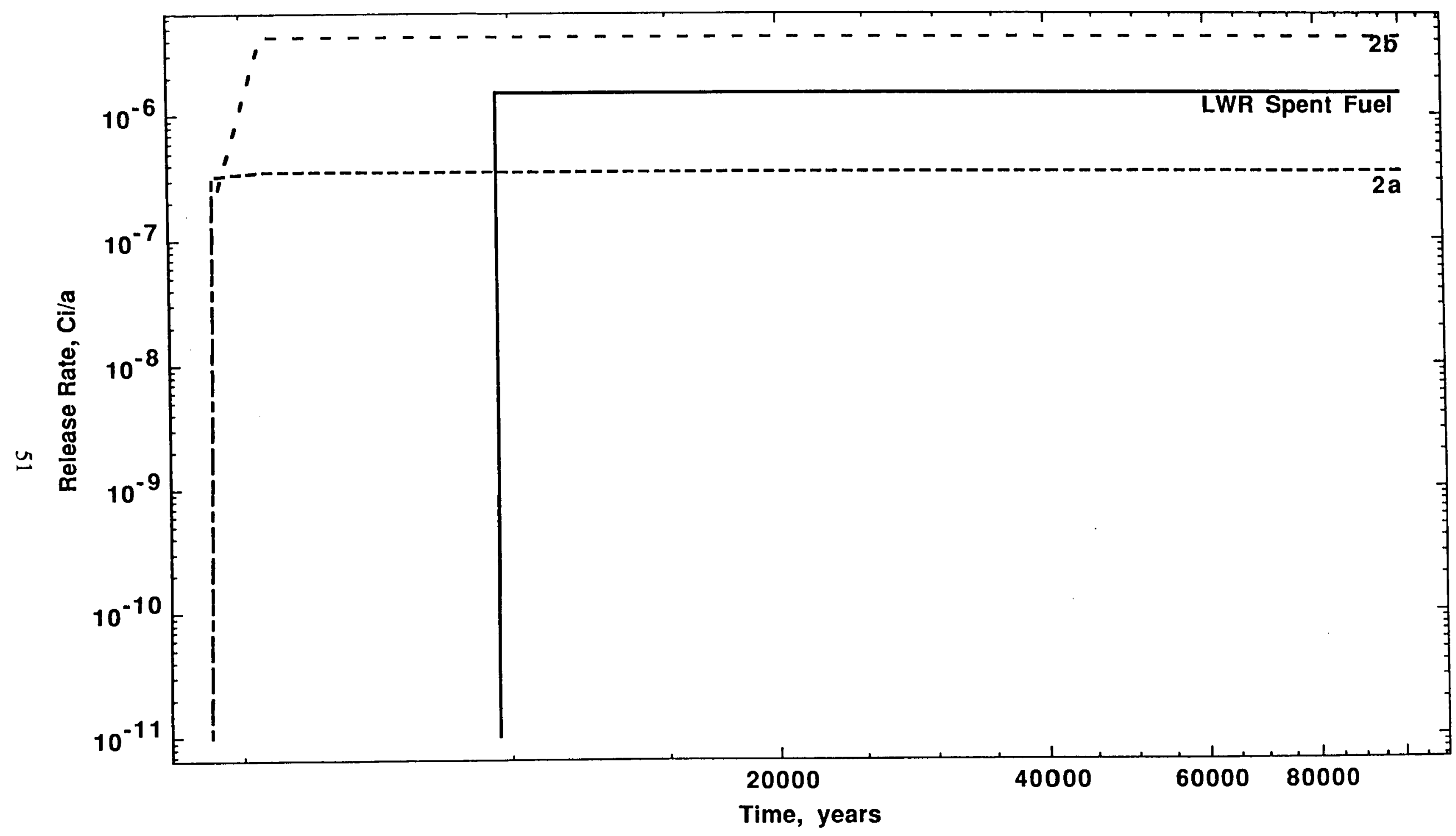

Figure 18. Release rates of Np-237 from Schemes $1,2 a$ and $2 b$ 


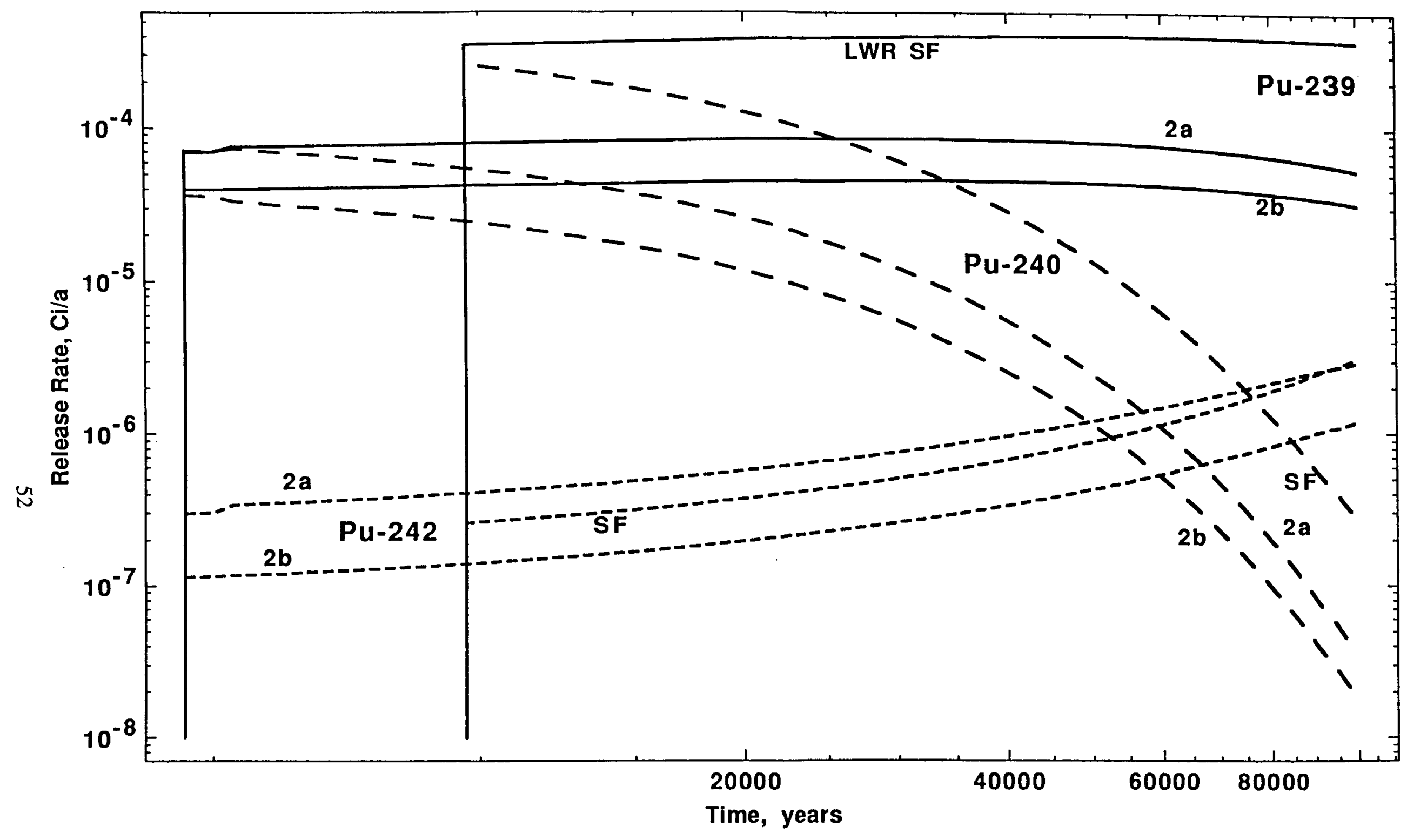

Figure 19. Release rates of Pu isotopes from Schemes $1,2 a$ and $2 b$ 


\section{References}

T. A. Abrajano, J. K. Bates, T. J. Gerding, and W. L. Ebert, 1988. "The Reaction of Glass During Gamma Irradiation in a Saturated Tuff Environment, Part III: Long Term Experiments at 104 rad/hr," ANL-88-14.

M. J. Apted, W. J. O'Connell, K. H. Lee, A. T. MacIntyre, T.-S. Ueng, T. H. Pigford and W. W.-L. Lee, 1991. "Preliminary Calculations of Release Rates of Tc-99, I-129, Cs-135, \& Np-237 from Spent Fuel in a Tuff Repository," Proc. of the 1991 International High-Level Radioactive Waste Management Conference, 1080 .

R. W. Barnard and H. A. Dockery, 1991. Technical Summary of the Performance Assessment Calculational Exercises for 1990, Volume 1. SAND 90-2726.

M. Benedict, T. H. Pigford and H. W. Levi, 1981. Nuclear Chemical Engineering, Second Edition, New York: McGraw-Hill.

W. J. Broad, 1990. "A Mountain of Trouble," New York Times Magazine, November 18, 1990.

C.J. Bruton, 1988. "Geochemical Simulation of Dissolution of West Valley and DWPF Glasses in J-13 Water at $90^{\circ}$ C." in Scientific Basis for Nuclear Waste Management XI, eds. M.J. Apted and R.E. Westerman, Materials Research Society, Pittsburgh, PA, 607.

L. L. Burger, R. D. Scheek and K. D. Wiemers, 1980. Selection of a Form for Fixation of I-129," PNL-4045.

International Atomic Energy Agency, 1982. Evaluation of Actinide Partitioning and Transmutation, Tech Rpt 214, Vienna, IAEA.

J. Hirschfelder, P. L. Chambré, W. W.-L. Lee, T. H. Pigford, and M. M. Sadeghi, 1991. "Effects of Actinide Burning on Waste Disposal at Yucca Mountain," Trans. Am. Nuc. Soc., 64, 111.

M. J. Lineberry and R. D, Phipps, 1989. "Preparations for the IFR Fuel Cycle Demonstration," Trans. Am. Nuc. Soc., 60, 170.

T. Priem, F. Bretheau \& A. Cernes, 1990. "Effect of Minor Actinide Removal from Fission Product Before Vitrification on the Radiological Impact of a High Level Waste Deep Repository," Proc. of the 1990 International High-Level Waste Management Conference, 1138.

M. M. Sadeghi, T. H. Pigford, P. L. Chambré and W. W.-L. Lee, 1990. Equations for Predicting Release Rates for Waste Packages in Unsaturated Tuff, LBL-29254.

M. Sadeghi, T. H. Pigford, P. L. Chambré and W. W.-L. Lee, 1991. Prediction of Release Rates for a Waste Repository at Yucca Mountain, LBL-27767.

C. R. Southwell, J. D. Bultman and A. L. Alexander, 1976. "Corrosion of Metals in Tropical EnvironmentFinal Report of 16-year Exposures," Materials Performance, 15, No. 7, 9.

M. Thompson, 1990. "Actinide Recycle in Advanced Liquid-Metal Reactors," Trans. Am. Nuc. Soc., 61, 301.

M. Thompson and I. N. Taylor, 1991. Projected Waste Packages Resulting from Spent Fuel Separation Processes, EPRI-NP-7262.

U.S. Department of Energy, 1987. "Characteristics of Spent Fuel, High-Level Waste, and Other Radioactive Wastes Which May Require Long-Term Isolation," DOE/RW-0184.

R. E. Wilems and J. G. Danna, 1991. The Effects of Transuranic Separation on Waste Disposal," EPRINP-7263.

C. N. Wilson, 1990. "Results from NNIVSI Series 3 Spent Fuel Dissolution Tests," PNL-7170.

C. N. Wilson and C. J. Bruton, 1989. "Studies on Spent Fuel Dissolution Behavior under Yucca Mountain Repository Conditions,” PNL-SA-16832. 


\section{Appendix}

Release rates, in fractional release rates and in curie/annum, are available for

\begin{tabular}{|l|c|c|c|c|c|c|c|c|c|c|}
\hline & $\mathrm{A} 1-1,2$ & $\mathrm{~A} 1-3$ & $\mathrm{~A} 1-4$ & $\mathrm{~A} 1-6$ & $\mathrm{~A} 1-7$ & $\mathrm{~A} 1-8$ & $\mathrm{~A} 3-2,5$ & $\mathrm{A3}-4$ & $\mathrm{~B} 1-2$ & $\mathrm{~B} 1-4$ \\
\hline $\mathrm{Am}-241$ & $\mathrm{x}$ & & & & & $\mathrm{x}$ & $\mathrm{x}$ & & $\mathrm{x}$ & $\mathrm{x}$ \\
\hline $\mathrm{Am}-243$ & $\mathrm{x}$ & & & & & $\mathrm{x}$ & $\mathrm{x}$ & & $\mathrm{x}$ & $\mathrm{x}$ \\
\hline $\mathrm{Cs}-135$ & & & $\mathrm{x}$ & & $\mathrm{x}$ & & $\mathrm{x}$ & & & $\mathrm{x}$ \\
\hline $\mathrm{I}-129$ & & $\mathrm{x}$ & & & & & & $\mathrm{x}$ & & \\
\hline $\mathrm{Np}-237$ & $\mathrm{x}$ & & & & & $\mathrm{x}$ & $\mathrm{x}$ & & $\mathrm{x}$ & $\mathrm{x}$ \\
\hline $\mathrm{Pu}-239$ & $\mathrm{x}$ & & & & & $\mathrm{x}$ & $\mathrm{x}$ & & $\mathrm{x}$ & $\mathrm{x}$ \\
\hline $\mathrm{Pu}-240$ & $\mathrm{x}$ & & & & & $\mathrm{x}$ & $\mathrm{x}$ & & $\mathrm{x}$ & $\mathrm{x}$ \\
\hline $\mathrm{Pu}-242$ & $\mathrm{x}$ & & & & & $\mathrm{x}$ & $\mathrm{x}$ & & $\mathrm{x}$ & $\mathrm{x}$ \\
\hline $\mathrm{Se}-79$ & & & & $\mathrm{x}$ & & $\mathrm{x}$ & & & & $\mathrm{x}$ \\
\hline $\mathrm{Tc}-99$ & $\mathrm{x}$ & & & $\mathrm{x}$ & & $\mathrm{x}$ & $\mathrm{x}$ & & $\mathrm{x}$ & $\mathrm{x}$ \\
\hline $\mathrm{U}-234$ & $\mathrm{x}$ & & & $\mathrm{x}$ & & $\mathrm{x}$ & $\mathrm{x}$ & & $\mathrm{x}$ & $\mathrm{x}$ \\
\hline $\mathrm{U}-238$ & $\mathrm{x}$ & & & $\mathrm{x}$ & & $\mathrm{x}$ & $\mathrm{x}$ & & $\mathrm{x}$ & $\mathrm{x}$ \\
\hline
\end{tabular}

Notes:

All files are ASCII. Files for curie/annum end with a ' $c$ '. 